\title{
Waste Isolation Pilot Plant Borehole Data
}

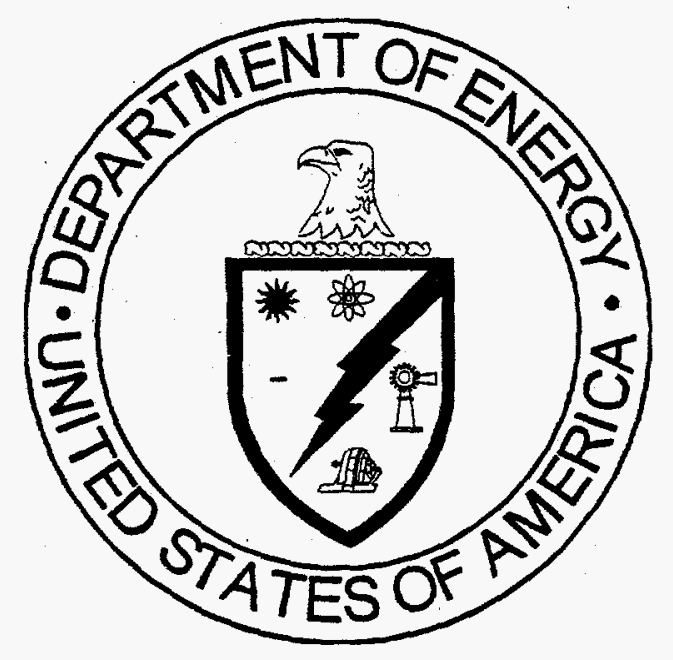

April 1995

Prepared for

United States Department of Energy Carlsbad Area Office

Westinghouse Electric Corporation

Waste Isolation Division 


\section{DISCLAIMER}

This document was prepared as an account of work sponsored by an agency of the United States Government. Neither the United States Government nor any agency thereof, nor any of their employees, makes any warranty, express or implied, or assumes any legal liability or responsibility for the accuracy, completeness, or usefulness of any information, apparatus, product or process disclosed, or represents that its use would not infringe privately owned rights. References herein to any specific commercial product, process, or service by trade name, trademark, manufacturer, or otherwise, does not necessarily constitute or imply its endorsement, recommendation, or favoring by the United States Government or any agency thereof. The views and opinions of authors expressed herein do not necessarily state or reflect those of the United States Government or any agency thereof.

This document has been reproduced directly from the best possible copy. It is available to DOE and DOE contractors at the following address:

Office of Scientific and Technical Information

P. O. Box 62

Oak Ridge, TN 37831

Prices available from (615) 576-8401

Available to the public from the National Technical Information Service

U. S. Department of Commerce 5285 Port Royal Road Springfield, VA 22161

Processing and final preparation of this report was performed by the Waste Isolation Pilot Plant Management and Operating Contractor for the U.S. Department of Energy under Contract No. DE-AC04-86AL31950. 


\section{DISCLAIMER}

Portions of this document may be illegible in electronic image products. Images are produced from the best available original document. 


\section{TABLE OF CONTENTS}

Section

Page

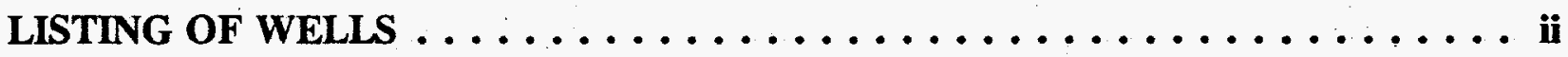

EXECUTIVE SUMMARY $\ldots \ldots \ldots \ldots \ldots \ldots \ldots \ldots \ldots \ldots \ldots \ldots \ldots \ldots$

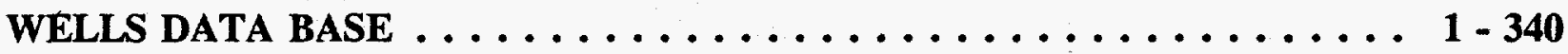

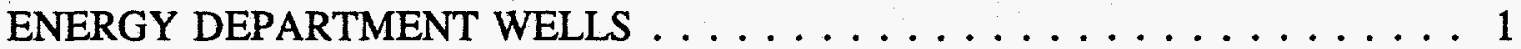

GEOLOGIC EXPLORATION BOREHOLES . . . . . . . . . . . . . . 27

HYDROLOGIC TEST BOREHOLES . . . . . . . . . . . . . . . . 68

POTASH BOREHOLES . . . . . . . . . . . . . . . . . . . . . . . . . . . . . . . . . . . . . .

COMMERCIALLY DRILLED POTASH BOREHOLES $\ldots \ldots \ldots \ldots$

SUBSURFACE EXPLORATION BOREHOLES $\ldots \ldots \ldots \ldots . \ldots \ldots 2$

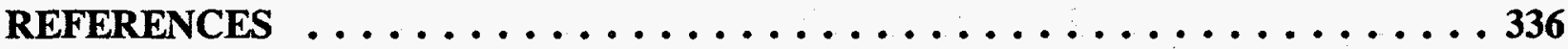




\section{LISTING OF WELLS}

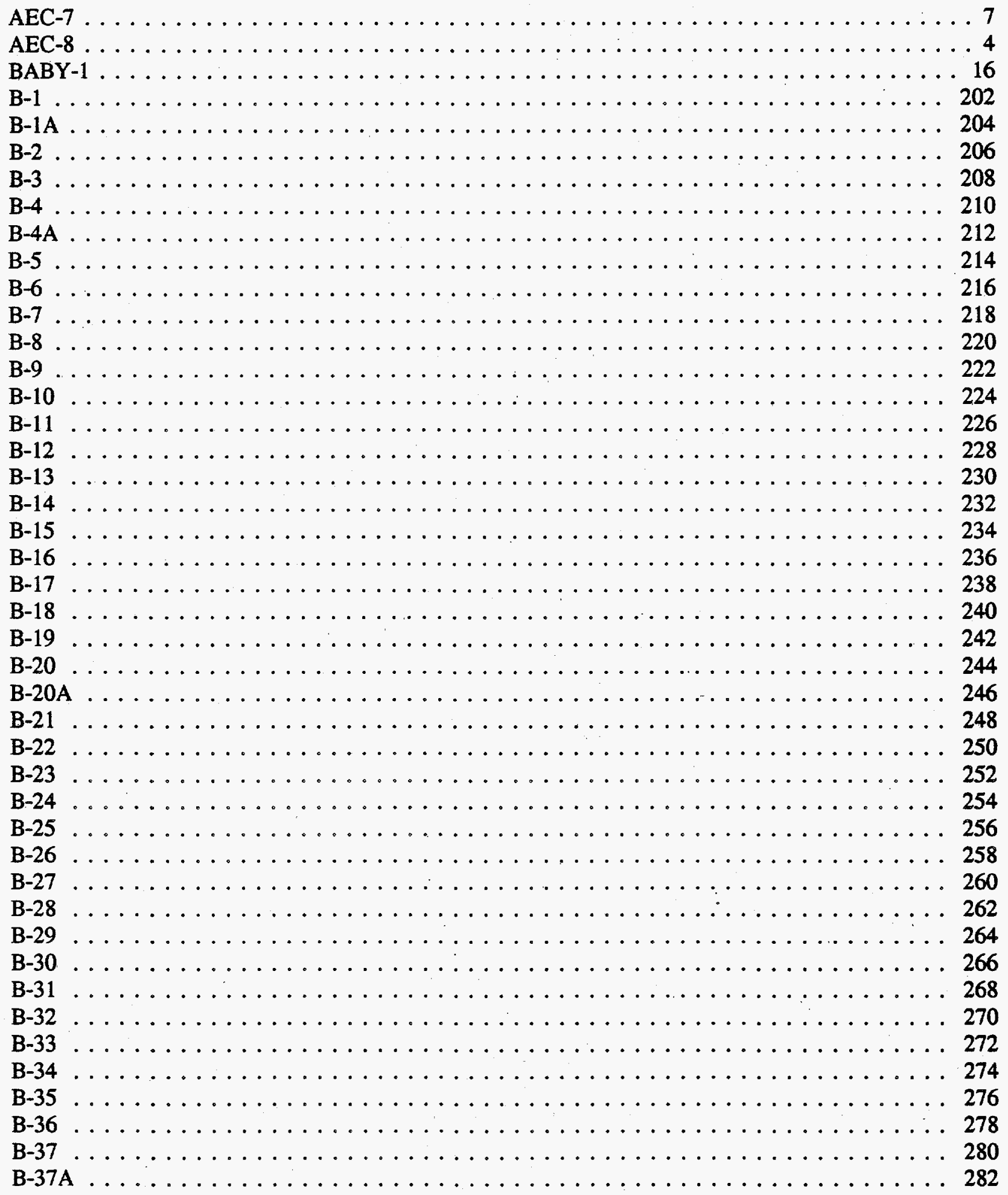




\section{LISTING OF WELLS (CONTINUED)}

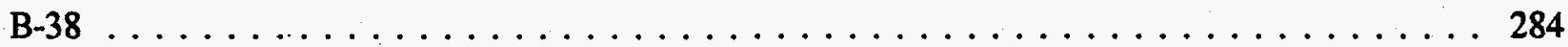

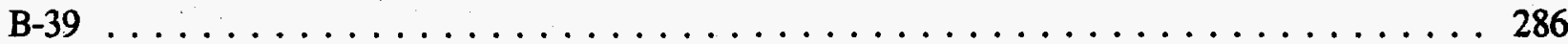

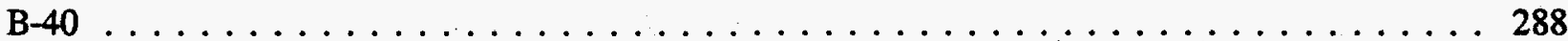

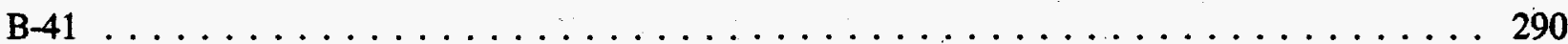

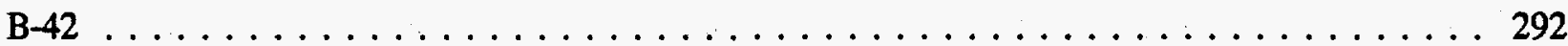

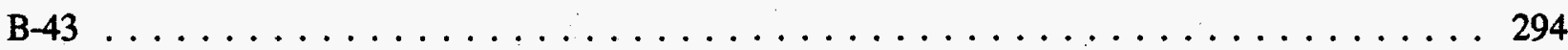

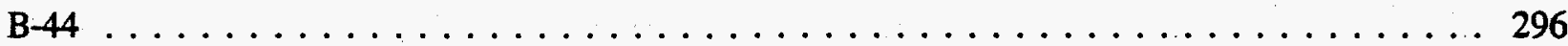

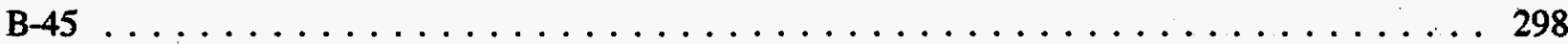

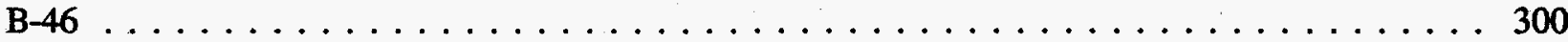

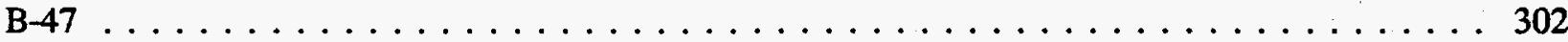

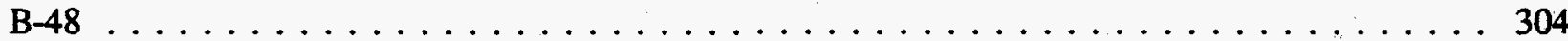

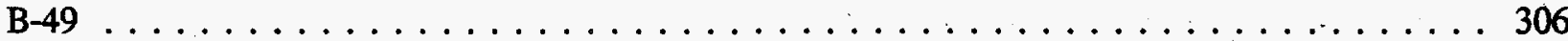

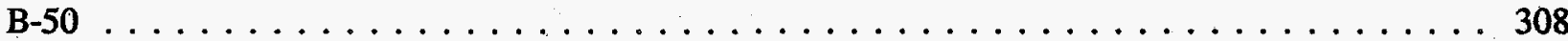

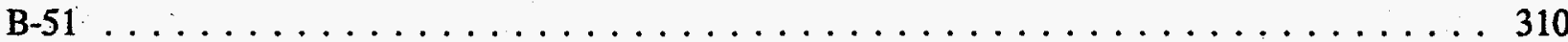

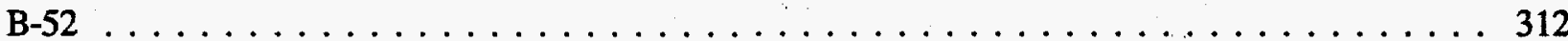

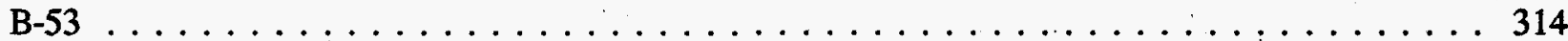

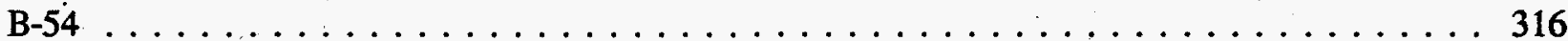

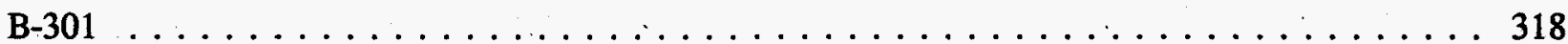

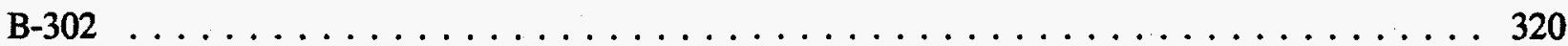

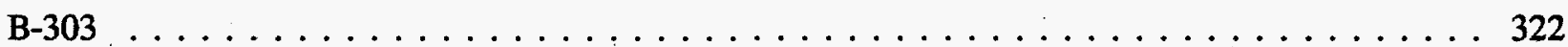



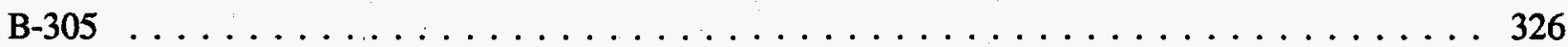

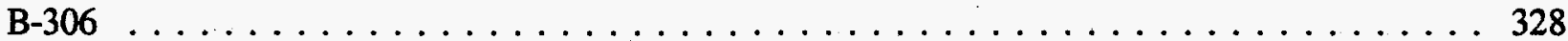

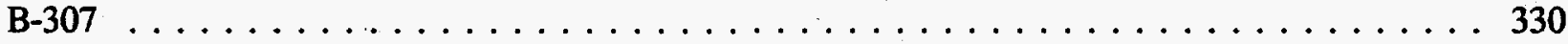

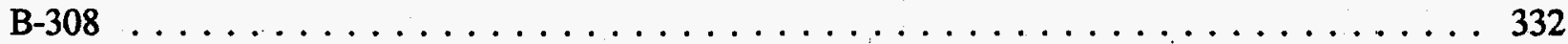

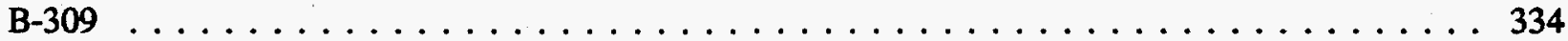

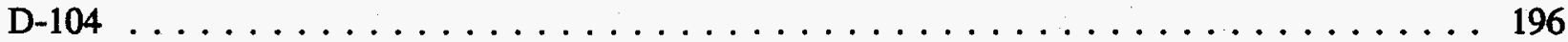

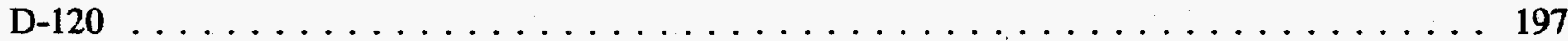

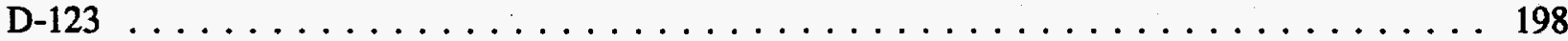

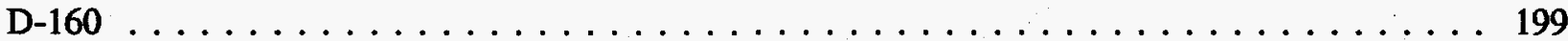

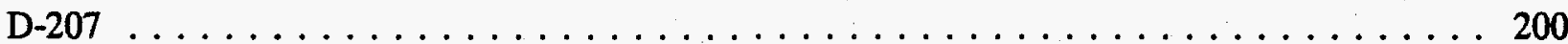

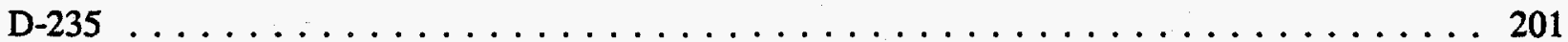

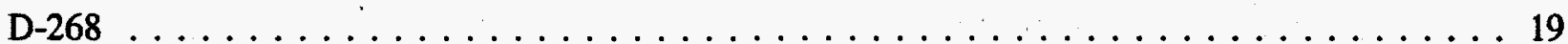

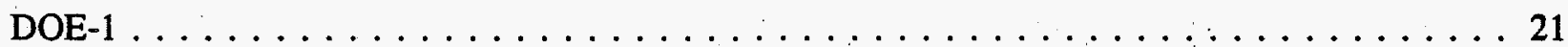

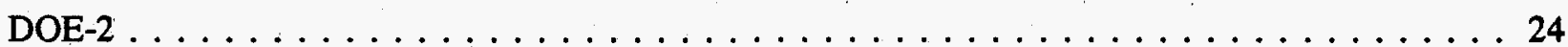

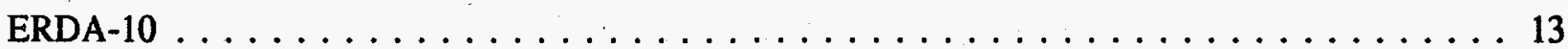

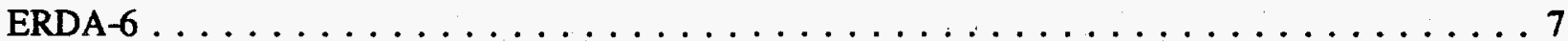

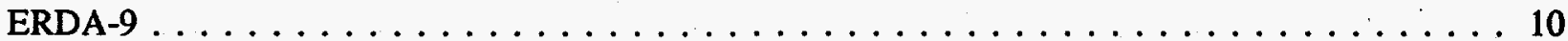

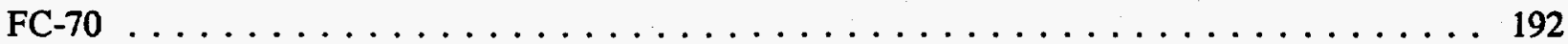

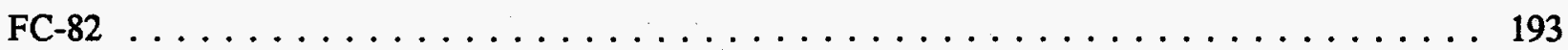

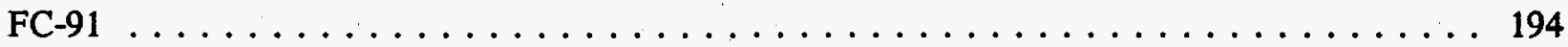

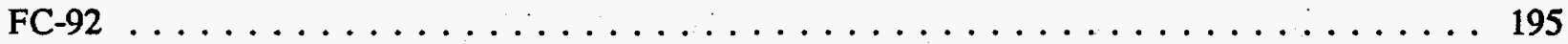

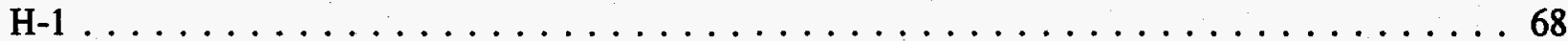

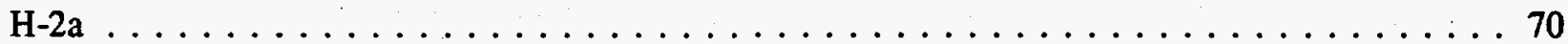

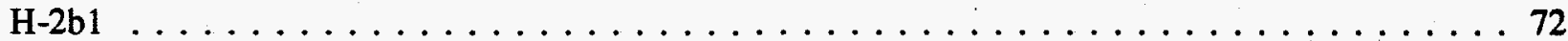




\section{LISTING OF WELLS (CONTINUED)}

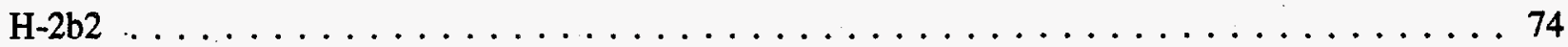

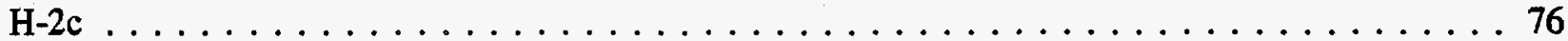

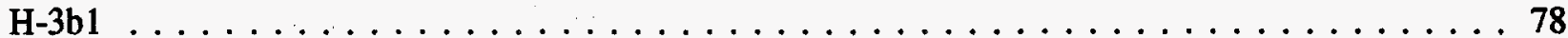

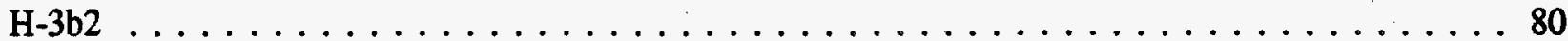

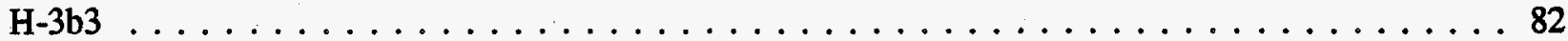

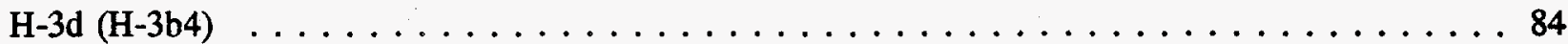

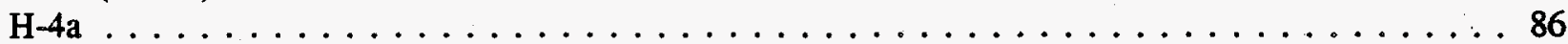

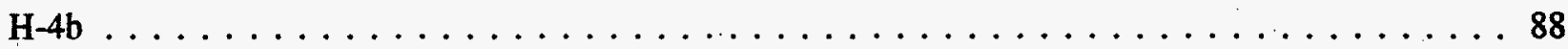

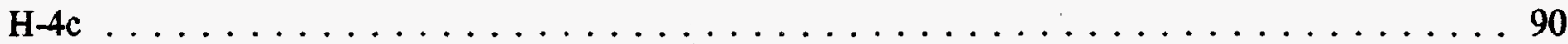

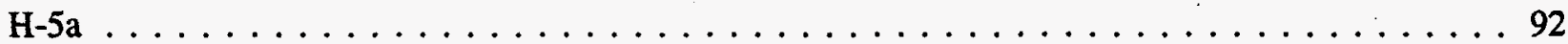

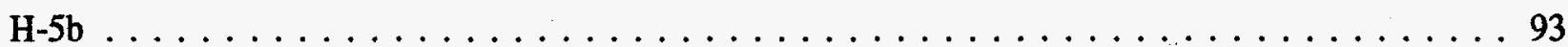

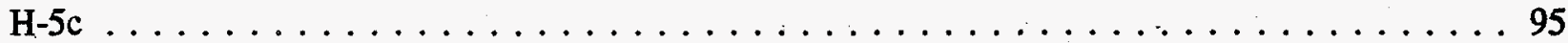

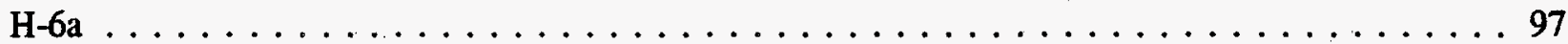

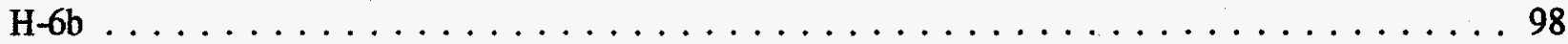

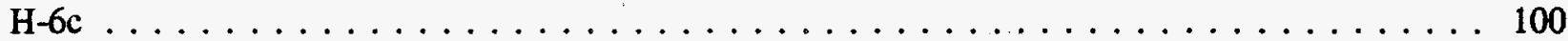

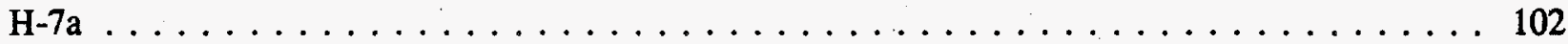

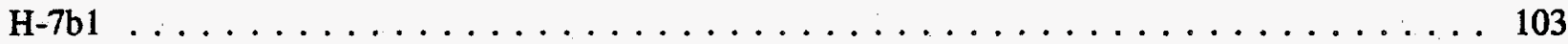

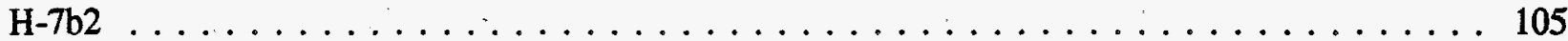

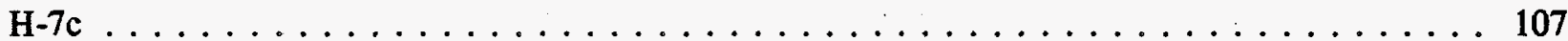

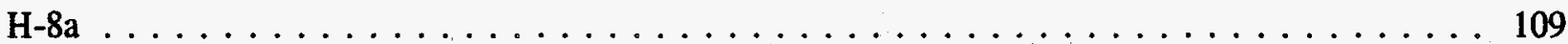

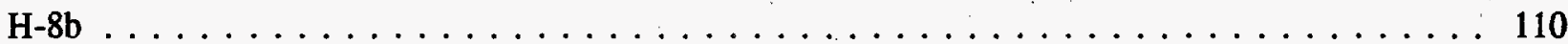

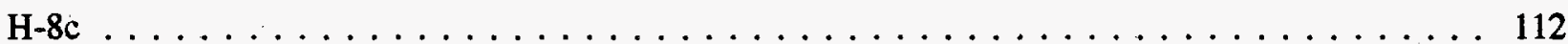

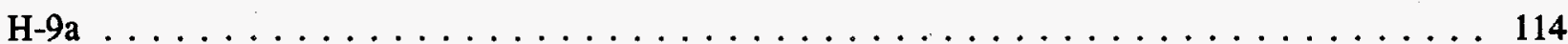

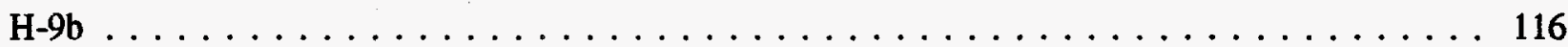

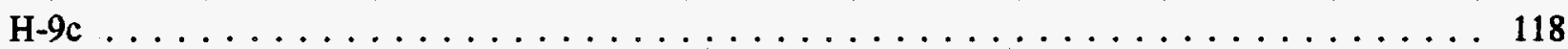

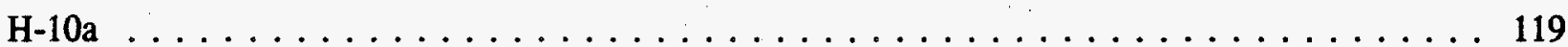

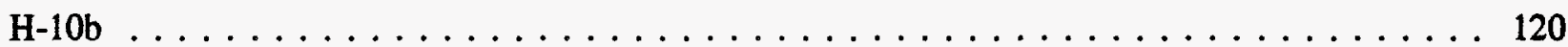

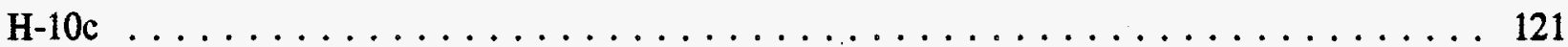

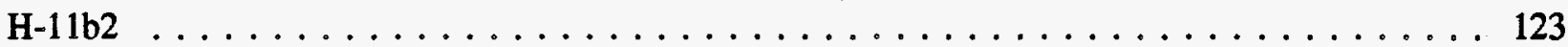

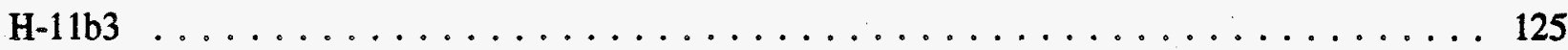

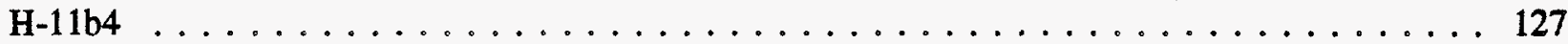

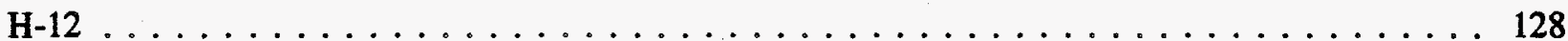

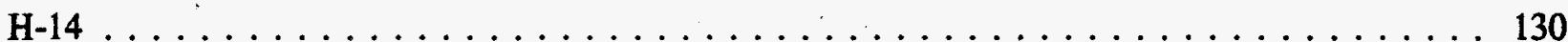

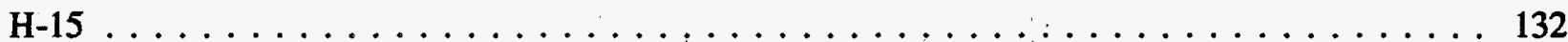

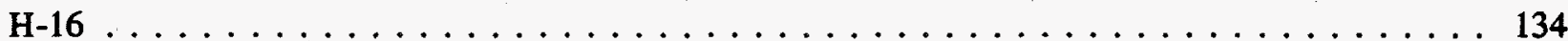

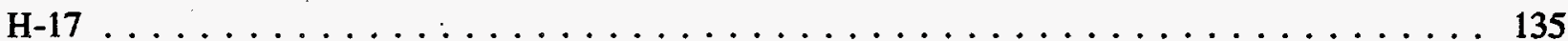

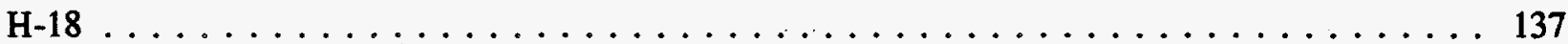

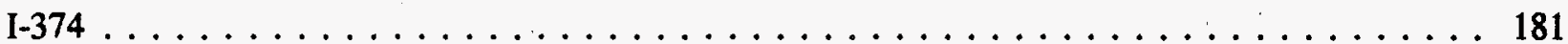

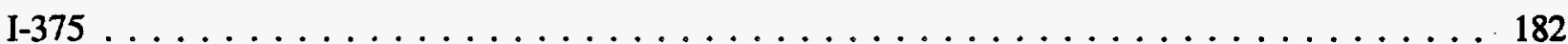

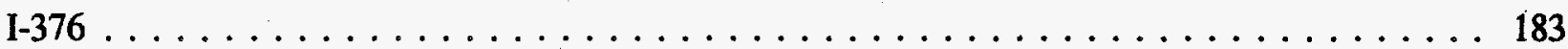

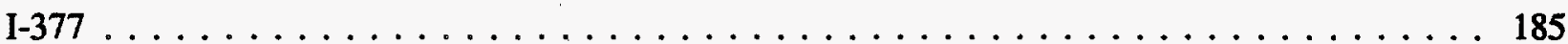

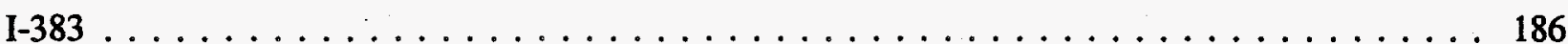

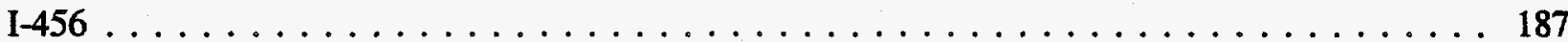

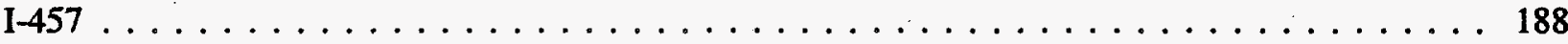

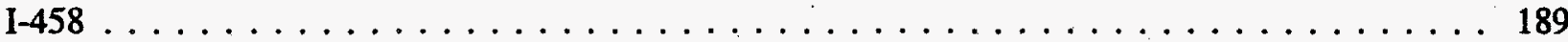




\section{LISTING OF WELLS (CONTINUED)}

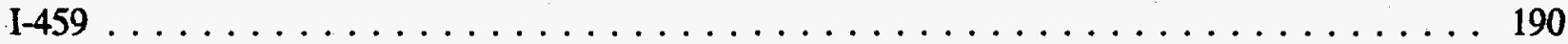

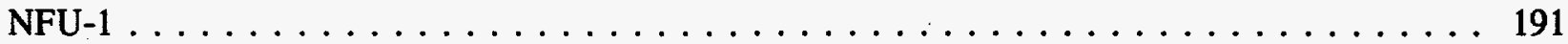

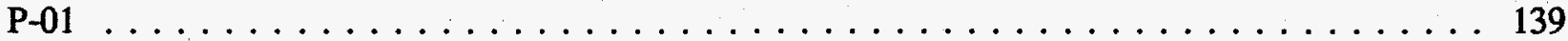

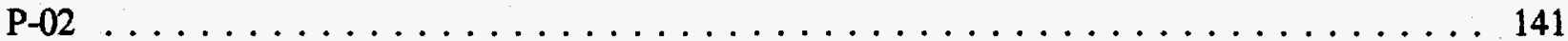

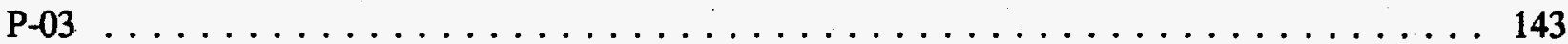

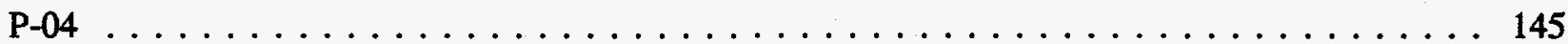

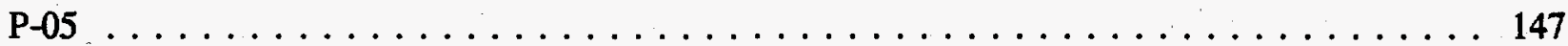

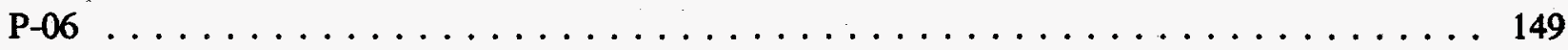

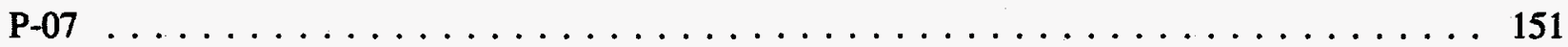

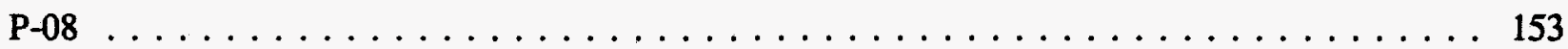

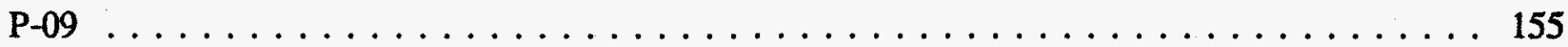

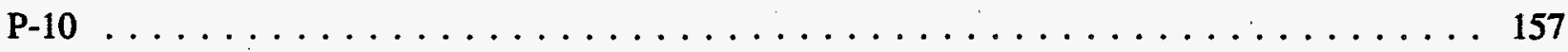

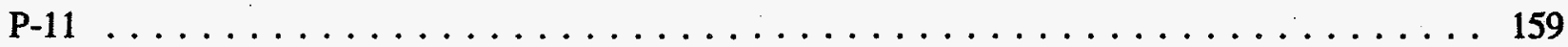

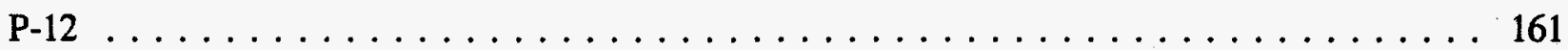

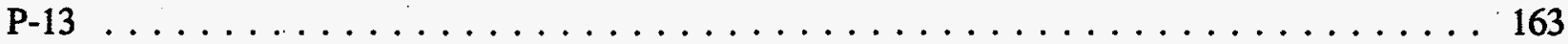

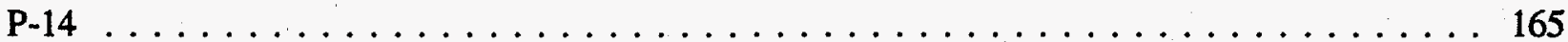

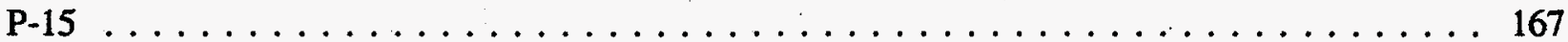

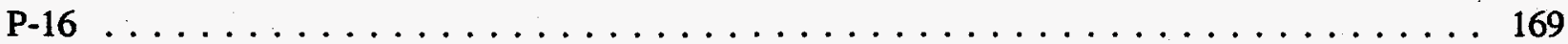

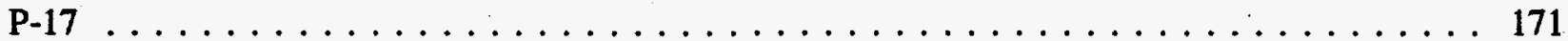

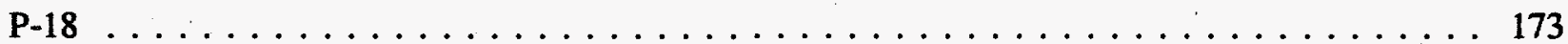

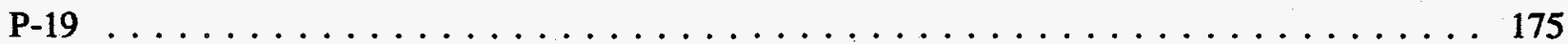

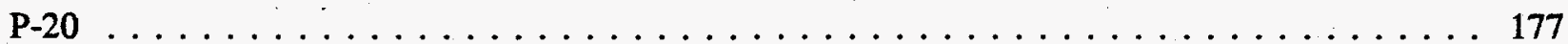

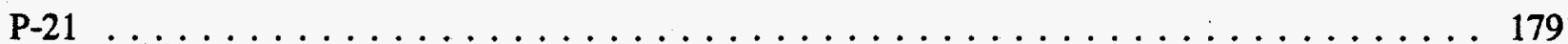

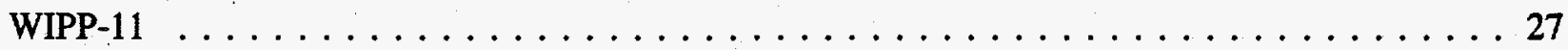

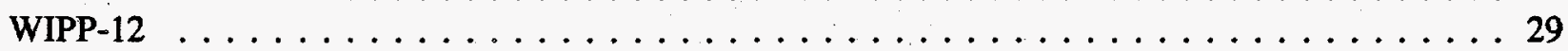

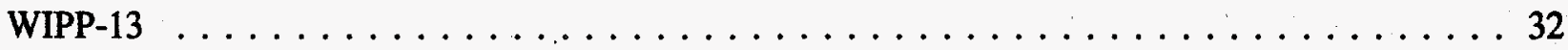

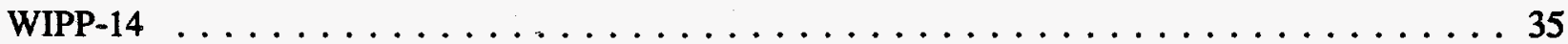

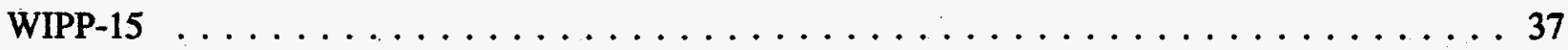

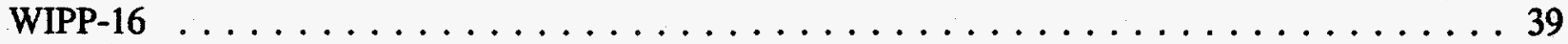

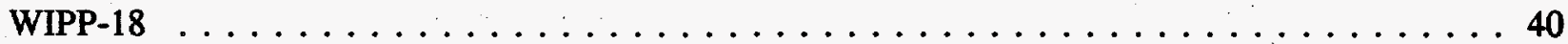

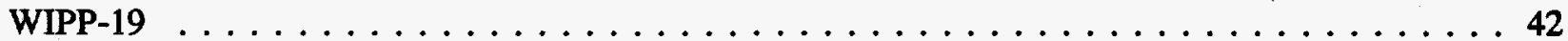

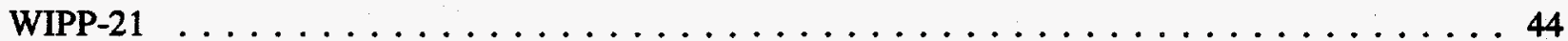

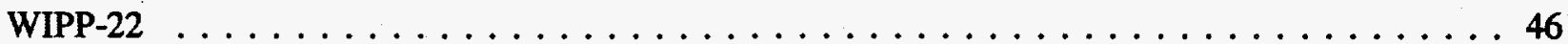

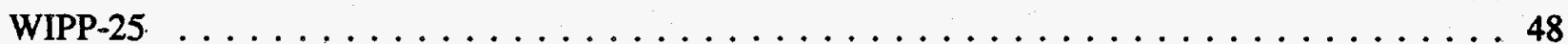

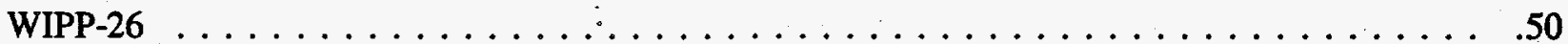

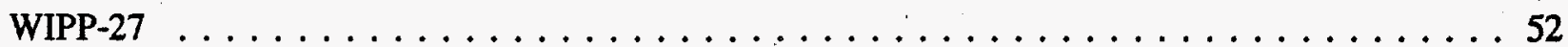

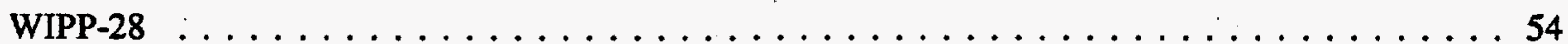

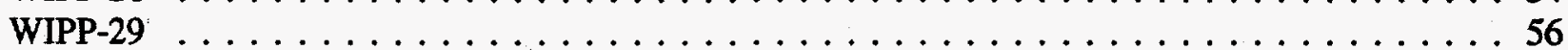

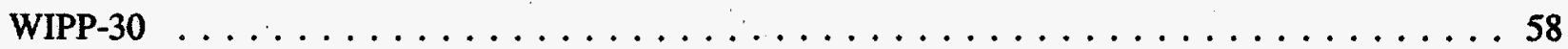

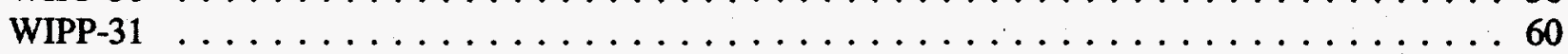

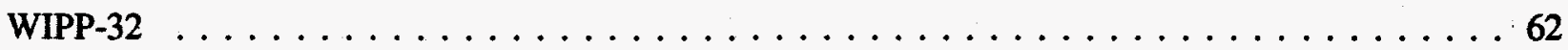

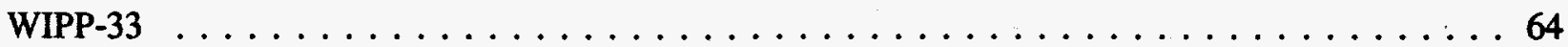

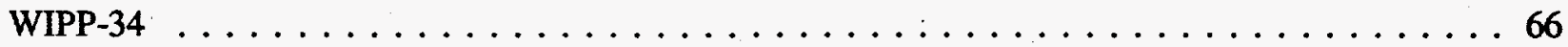




\section{EXECUTIVE SUMMARY}

Data pertaining to all the surface boreholes that have been used at the WIPP site for site characterization hydrological testing and resource evaluation exist in numerous source documents. This project was initiated to develop a comprehensive data base that would include the data on all WIPP related surface boreholes from the Atomic Energy Commission, Waste Isolation Pilot Plant Energy Research and Development Administration, Department of Energy, and Hydrologic Test Borehole Programs.

The data compiled from each borehole includes: the operator, permit number, location, total depth, type of well, driller, drilling record, casing record, plugging schedule, and stratigraphic summary. There are six groups of boreholes contained in this data base, they are as follows: Commercially Drilled Potash Boreholes, Energy Department Wells, Geologic Exploration Boreholes, Hydrologic Test Boreholes, Potash Boreholes, and Subsurface Exploration Boreholes.

There were numerous references which contained borehole data. During the compilation of this data base, it was noted that in some cases the data found in one document was inconsistent with data in another document. In order to ensure consistency and accuracy in the data base, the same references were used for as many of the boreholes as possible. For example, all elevations and locations were taken from Compilation and Comparison of Test-Hole Location Surveys in the Vicinity of the WIPP Site. SAND 88-1065, Table 3-5. There are some sections where a data field is left blank. In this case, the information was either not applicable or was unavailable.

The attached reference list is divided into two categories: major references and minor references. The major references are the references most frequently used to obtain data for this document. Minor references were primarily single borehole reference reports.

All the data included in this document has been reviewed by an independent third party and verified as to its accuracy and completeness. 
BOREHOLE: OFERATOR:

PERMIT NO.:

LOCATION:

ELEVATION:

TOTAL DEPTH:

TYPE OF WELL:

DRILLER:

DRILLING RECORD:
AEC-7

Sandia National Labs

0.08 .1323

2036.97' FNL, 2033.23' FEL

Sec. 31, T 21 S, R 32 E

3657.25' (Top of Casing)

$4734^{\prime}$

Geological Exploration

Verna Drilling Company

Date Started: $\quad 03 / 20 / 74$
Date Completed: $04 / 19 / 80$

\section{CASING RECORD:}

Diameter: $133 / 8$

Grade: $\mathrm{H}-40$

WiFt: 48

From: 0

To: 40

Cement:

Diameter: $85 / 8$

Grade: $\mathrm{H}-40$

Wt/Ft: 28

From: 0

To: 1016

Cement:

PLUGGING SCHEDULE:

No plugging data.

STRATIGRAPHIC SUMMARY: Attached 
STRATIGRAPHIC SUMMARY

AEC-7

Quaternary

Holocene-Eolian Sand

Mescalero Caliche

Triassic

Santa Rosa Sandstone

Permian

Dewey Lake Red Beds

Rustler Formation

Magenta Dolomite Member

Culebra Dolomite Member

Salado Formation

Upper Member

MB 101

MB 102

MB 103

MB 104

MB 105

MB 106

MB 107

MB 108

MB 109

MB 111

MB 112

MB 114

MB 116

McNutt Potash Zone

Vaca Triste Sandstone

MB 117

MB 118

MB 119

MB 121

MB 122

Union Anhydrite

MB 123

MB 124

MB 126

Lower Member

MB 128

MB 129

MB 131

MB 132

MB 133

MB 134

MB 136

MB 139

MB 140

MB 141

MB 142

MB 143

Cowden Anhydrite
16.2-20.2

20.2-24.2

$24.2-133.0$

133.0-675.0

675.0-1000.6

$733.5-767.0$

872.2-900.5

1000.6-3014.7

1000.6-1505.1

1125.8

1158.5

1171.4-1186.5

1197.0

1211.5

1230.5

1269.0

1278.0

1303.8-1324.5

1378.0

1397.5

1450.5

1498.4

1505.1-1881.0

1505.1-1514.1

1578.9

1595.6

1619.8

1661.5

1668.5

1696.2-1705.0

1774.8-1781.2

1785.5-1795.5

1881.0

1881.0-3104.7

1918.0

1943.9

2013.5

2039.0

2057.5

2097.0-2109.0

2161.0-2168.0

2267.5

2302.8-2314.2

2364.5

2400.2-2406.5

2453.0-2455.6

2520.0-2539.0 


\section{STRATIGRAPHIC SUMMARY}

AEC-7

Castile Formation

Anhydrite III

Halite II

Anhydrite II

Halite II

Anhydrite II

Halite I

Anhydrite I

Bell Canyon Formation

Reef Talus

Lamar Limestone

Ramsey Sandstone

Ford Shale

Olds Sandstone
3014.7-4535.3

3014.7-3113.0

$3113.0-3310.0$

3310.0-3506.9

3506.9-3588.2

3588.2-4055.0

4055.0-4182.3

4182.3-4535.5

$4535.5-4731.9$ (T.D.)

4535.5-4584.7

4584.7-4633.3

4633.3-4678.5

4678.5-4714.9

4714.9-4731.9 
BOREHOLE: OPERATOR:

PERMIT NO.:

LOCATION:

ELEVATION:

TOTAL DEPTH:

TYPE OF WELL:

DRILLER:

DRILLING RECORD:

\author{
AEC-8 \\ Sandia National Labs \\ Unkown \\ 935' FNL, 1979' FWL \\ Sec. 11, T 22 S, R 31 E \\ 3532' (Top of Casing) \\ 4922' \\ Geological Exploration \\ Sonora Drilling Company
}

Date Started: $\quad 06 / 28 / 76$
Date Completed: $\quad 08 / 05 / 76$

\section{CASING RECORD:}

Diameter: $133 / 8$

Grade:

$\mathrm{Wt} / \mathrm{Ft}: 54.5$

From: 0

To: 42

Cement:

Diameter: $85 / 8$

Grade:

$\mathrm{Wt} / \mathrm{Ft}: 28$

From: 0

To: 885

Cement: $660 \mathrm{Cu}$. Ft.

Diameter: $51 / 2$

Grade: J-55

Wt/Ft: 15.5

From: 0

To: 4919

Cement: $2481 \mathrm{Cu}$. Ft.

Note: Completed logging. Ran 7 7/8" bit in the hole and conditioned mud to run casing. Corrected total depth to 4922'. Laid down drill pipe. Ran 123 joints (4933.72') of 51/2" O.D., 15.50\#, J-55, range 3, ST\&C casing in the hole and landed at 4918.77' (4907.22' GL). A Halliburton guide shoe was on bottom with a float shoe at $4859.52^{\prime}$ GL. Centralizers were placed at $4905^{\prime}$ GL, $4827^{\prime}$ GL, 800' GL and 50' GL. 38 joints (1533.25') of casing were sand blasted for better bond from 2374' to 4907' KB.

Completed running casing. Cemented annulus using Halliburton with 1500 sacks (2085 cu ft) of 50-50 Pozmix " $\mathrm{C}$ " with 9.7 \#/sk of salt and $2 \%$ bentonite followed by 300 sacks ( $396 \mathrm{cu} \mathrm{ft}$ ) of Class "C" cement. Displaced cement with 117 barrels of water. Casing was reciprocated during displacement. After displacing 90 barrels approximately 2 barrels of cement circulated to the surface, circulation was lost at this point. Bumped plug with 2000 psi and held. Cement in place at 0220 hours. Ran Dresser Atlas temperature log, top of cement at $880^{\prime}$. Ran Sperry-Sun gyroscopic multishot survey in the hole on $25^{\prime}$ stations and $100^{\prime}$ stations out of the hole.

All depths are measured from Kelly Bushing $11.5^{\prime}$ above ground level.

\section{PLUGGING SCHEDULE:}

See Casing Record. 
STRATIGRAPHIC SUMMARY

BOREHOLE

AEC-8
ROCK UNIT

Quaternary: Holocene-Eolian Sand

Mescalero Caliche

Triassic

Santa Rosa Sandstone

Permian

Dewey Lake Red Beds

Rustler Formation

Magenta Dolomite Mem

Culebra Dolomite Mem

Salado Formation

Upper Member

MB 101

MB 102

MB 103

MB 104

MB 105

MB 106

MB 107

MB 108

MB 109

MB 111

MB 112

MB 113

MB 114

MB 115

MB 116

McNutt Potash Zone

Vaca Triste Sands

MB 117

MB 118

MB 119

MB 120

MB 121

MB 122

Union Anhydrite

MB 123

MB 124

MB 126

Lower Member

MB 127

MB 128

MB 129

MB 130

MB 131

MB 132

MB 133

MB 134

MB 135

MB 136

MB 137
DEPTH INTERVAL IN FEET

9.0-29.0

29.0-35.0

$35.0-177.4$

$177.4-668.0$

$668.0-990.0$

727.3-749.5

848.3-873.3

$990.0-2979.6$

990.0-1469.3

1101.0-1116.5

1146.7

1157.7-1173.7

1184.4

1197.6

1215.3

1240.5

1261.3

1286.1-1304.7

1351.9

1370.4

1398.7

1418:2

1451.3

1462.5

1469.3-1826.5

1469.3-1484.1

1535.4

1557.0

1580.5

1603.2

1622.0

1628.6

1648.0-1657.9

1733.5

1746.8

1826.5

1826.5-2979.6

1849.5

1861.5

1885.3

1894.7

1959.6

1989.0

2005.5

2047.9-2060.5

2076.0

2114.0-2128.3

2138.5 
STRATIGRAPHIC SUMMARY

BOREHOLE

AEC-8
ROCK UNIT

MB 138

MB 139

MB 140

MB 141

MB 142

MB 143

MB 144

Cowden Anhydrite

Castile Formation

Anhydrite III

Halite II

Anhydrite II

Halite I

Anhydrite I

Bell Canyon Formation

Lamar Limestone

Ramsey Sand

Ford Shale
DEPTH INTERVAL IN FEET

2187.4

2247.8

2309.1-2316.5

2369.1

2411.8-2419.6

2465.2-2472.7

2498.8-2510.9

2539.5-2561.6

2979.6-4315.0

2979.6-3290.0

3290.0-3555.0

3555.0-3695.5

3695.5-4038.0

4038.0-4315.0

4315.0-4918.0 (T.D.)

4344.5-4374.0

4374.0-4436.0

4436.0-? 
BOREHOLE:

OPERATOR:

PERMIT NO.:

LOCATION:

ELEVATION:

TOTAL DEPTH:

TYPE OF WELL:

DRILLER:

DRILLING RECORD:
ERDA-6

Sandia National Labs

Unkown

2152' FSL, 910' FEL

Sec. 35, T 21 S, R 31 E

3540.2' (Top of Casing)

$2775^{\prime}$

Geologic Exploration

Boyles Bros. Drilling Company: 06/11/75 to 08/15/75 - Pan AM $工$

Date Started: $\quad 06 / 13 / 75$

Date Completed: 09/23/75

CASING RECORD:

Diameter: $133 / 8$

Grade:

Wt/Ft: 54.5

From: 0

To: 30

Cement: Ready Mix

Diameter: $85 / 8$

Grade: K-55

$\mathrm{Wt} / \mathrm{Ft}: 24$

From: 0

To: 880

Cement: 575 SX.

Note: Plugged back $2773^{\prime}$ to $2560^{\prime}$ with 225 cement. Hole filled with brine.

PLUGGING SCHEDULE:

See Casing Record.

STRATIGRAPHIC SUMMARY: Attached 


\section{STRATIGRAPHIC SUMMARY}

Quaternary

Holocene-Eolian Sand

Mescalero Caliche

Triassic

Santa Rosa Sandstone

\section{Permian}

Dewey Lake Red Beds

Rustler Formation Dissolution Residue

Magenta Dolomite Member

Dissolution Residue

Culebra Dolomite Member

Dissolution Residue

Salado Formation

Upper Unit

MB 100

MB 101

MB 102

MB 103

MB 104

MB 105

MB 106

MB 107

MB 108

MB 109

MB 110

MB 111

MB 112

MB 113

MB 114

MB 115

MB 116

McNutt Potash Zone

Vaca Triste Sandstone

11th Ore Zone

MB 117

MB 118

MB 119

10th Ore Zone

MB 120

9th Ore Zone

MB 121

MB 122

8th Ore Zone

Union Anhydrite

7th Ore Zone

6th Ore Zone

5th Ore Zone

MB 123

\section{0-9 \\ 9-17}

17-72

$72-538$

$538-811$

570-581

598-623

696-707

713-739

742-762

811-2396.5

811-1276.6

NR

923.5-929.9

956.8-958.2

970.3-984.3

992.1-992.4

NR

NR

1060.0-1060.9

1069.1-1069.9

1090.9-1113.5

NP

1161.5-1161.8

1178.9-1180.2

NP

1224.9-1226.9

1256.1-1257.2

1268.7-1271.4

1276.6-1612.9

1276.6-1287.3

1324.7-1329.8

1340.7-1342.7

1346-1349.0

1359.0-1366.2

1378.4-1379.4

1386.1-1395.5

1401.0-1402.3

1403.2-1410.3

1413.3-1415.5

1422.3-1424.3

1424.9-1437.0

1444.6-1453.8

1464.0-1468.5

$1479.7-1482.2$

1487.8-1494.9

1517.7-1524.9 


\section{ERDA-6}

\begin{abstract}
MB 124
4th Ore Zone

3rd Ore Zone

2nd Ore Zone

MB 125

1st Ore Zone
\end{abstract}

MB 126

Lower Member

MB 127

MB 128

MB 129

MB 130

MB 131

MB 132

MB 133

MB 134

MB 135

MB 136

MB 137

MB 138

MB 139

MB 140

MB 141

MB 142

MB 143

MB 144

Cowden Anhydrite

Castile Formation

Halite II

Anhydrite II

Halite I

Cowden Anhydrite

Castile Formation
1529.4-1537.5

1541.9-1549.1

1549.7-1553.0

$1555.5-1567.2$

1571.0-1574.3

NP

1587.0-1603.3

NP

1612.9-2396.5

1635.0-1635.7

$1647.7-1648.5$

1670.0-1671.5

NP

1743.0-1743.7

1770.6-1771.4

1785.6-1789.2

1833.2-1843.4

1860.4-1861.5

1900.5-1910.5

NP

1967.5-1967.7

2019.5-2022.4

2060.4-2075.6

2124.5-2126.6

2163.8-2169.5

2212.7-2215.6

2237.0-2237.7

2269.5-2291.0

2400.5-2775.0

2400.5-2555.1

2555.1-2732.5 (Fault or Rupture Zone)

2732.5-2775.0

2540.0-2653.0

2836.0-2889.0 
BOREHOLE:

OPERATOR:

PERMIT NO.:

LOCATION:

ELEVATION:

TOTAL DEPTH:

TYPE OF WELL:

DRILLER:

DRILLING RECORD:

\section{ERDA-9}

Sandia National Labs

Unknown

267.17' FSL, 176.74' FEL

Sec. 20, T 22 S, R 31 E

$3410.10^{\prime}$ (Top of Casing)

$2886^{\prime}$

Geologic Exploration

Sonora Drilling Company

Date Started: $\quad 04 / 28 / 76$
Date Completed: 06/26/76

\section{CASING RECORD:}

Diameter: 16

Grade:

$\mathrm{Wt} / \mathrm{Ft}$ :

From: 0

To: 40

Cement: $95 \mathrm{Cu}$. Ft.

Diameter: $103 / 4$

Grade: J-55

Wt/Ft: 40.5

From: 0

To: 1045

Cement: $1159 \mathrm{Cu}$. Ft.

Diameter: 7

Grade: J-55

Wt/Ft: 23

From: 0

To: 2883

Cement: Mud Pack

Note: Ran Dresser Atlas caliper log. Ran 79 joints (2889.66') of 7" O.D., 23\#.casing with a Dowell swirl type shoe on bottom and a Dowell orifice fill collar on top of the bottom joint. Set casing at $2882.66^{\prime}$ with centralizers at $2868^{\prime}, 2520^{\prime}$, and $1030^{\prime}$. Rigged up Dowell and pumped in 140 barrels of Baroid casing pack mud. Cemented annulus with 10 barrels of water, 12 barrels of chemical wash, 12 barrels of oil base slurry ahead of $122 \mathrm{cu}$. $\mathrm{ft}$. (115 sacks) of Class "H" cement $+3 \%$ calcium chloride. Seated plug with $1000 \mathrm{psi}$, plug holding. Cement in place at 1445 hours.

All depths are measured from Kelly Bushing 11.5' above ground level.

PLUGGING SCHEDULE:

See Casing Record.

STRATIGRAPHIC SUMMARY: Attached 
STRATIGRAPHIC SUMMARY

Kelly Bushing (KB) to Land Surface (LS)

Holocene Deposits

Pleistocene Rocks

Mescalero Caliche

Gatuna Formation

Triassic

Santa Rosa Sandstone

Permian

Dewey Lake Red Beds

Rusiter Formation

Dissolution Residue

Magenta Dolomite Member

Dissolution Residue

Culebra Dolomite Member

Dissolution Residue

Salado Formation

Upper Member

MB 100

MB 101

MB 102

MB 103

MB 104

MB 105

MB 106

MB 107

MB 108

MB 109

MB 110

MB 111

MB 112

MB 113

MB 114

MB 115

MB 116

McNutt Potash Zone

Vaca Triste Sands

11th Ore Zone

MB 117

MB 118

MB 119

10th Ore Zone

MB 120

9th Ore Zone

MB 121

MB 122

8th Ore Zone

Union Anhydrite

7 th Ore Zone

6th Ore Zone

5th Ore Zone
$0-12.0$

$12.0-22.0$

22.0-27.0

$27.0-54.0$

$54.0-63.0$

63.0-550.0

$550.0-860.0$

580.0-592.0

608.0-632.0

691.0-710.0

$716.0-739.0$

742.0-748.0

$860.0-2836.0$

$860.0-1362.0$

939.0

984.0

1026.0

1040.0-1050.0

1061.0

1075.0

1093.0-1094.0

1132.0

1142.0

1165.0-1188.0

NP

1238.0

1256.0-1258.0

1282.0-1284.0

1306.0

1340.0-1344.0

$1354.0-1356.0$

1362.0-1742.0

1365.0-1367.0

1420.0-1422.0

1431.0-1433.0

1455.0-1463.0

1482.0

1487.0-1493.0

1501.0-1502.0

1507.0-1512.0

1515.0-1517.0

1524.0

1531.0-1542.0

$1549.0-1557.0$

1572.0-1576.0

1590.0-1593.0

1597.0-1603.0 


\section{STRATIGRAPHIC SUMMARY}

MB 123

MB 124

4th Ore Zone

3rd Ore Zone

2nd Ore Zone

MB 125

1st Ore Zone

MB 126

Lower Member

MB 127

MB 128

MB 129

MB 130

MB 131

MB 132

MB 133

MB 134

MB 135

MB 136

MB 137

MB 138

MB 139

MB 140

MB 141

MB 142

MB 143

MB 144

Cowden Anhydrite

Castile Formation
1630.0-1637.0

1645.0-1653.0

1659.0-1669.0

$1676.0-1688.0$

1696.0-1698.0

NP

1712.0-1723.0

1742.0

1742.0-2836.0

1768.0-1770.0

1778.0-1781.0

1803.0-1805.0

1815.0

1884.0

1914.0-1915.0

1933.0-1935.0

1976.0-1989.0

2006.0

2043.0-2058.0

2075.0

2120.0-2121.0

2177.0-2180.0

2241.0-2251.0

2320.0-2330.0

2377.0-2391.0

2450.0-2456.0

2493.0-2506.0

2540.0-2653.0

2836.0-2889.0 
BOREHOLE:

OPERATOR:

PERMIT NO.:

LOCATION:

ELEVATION:

TOTAL DEPTH:

TYPE OF WELL:

DRILLER:

DRILLING RECORD:
ERDA-10

Sandia National Labs

Unkown

200' FNL, 2327' FEL

Sec. 34, T 23 S, R 30 E

3371.2' (Top of Casing)

4418.5'

Geologic Exploration

Corel Drilling Company

Date Started: $\quad 08 / 18 / 77$
Date Completed: $10 / 14 / 77$

\section{CASING RECORD:}

Diameter: $133 / 8$

Grade: $\mathrm{H}-40$

$\mathrm{Wt} / \mathrm{Ft}: 48$

From: 0

To: 50

Cement: $81 \mathrm{Cu}$. Ft.

Diameter: $95 / 8$

Grade: J-55

Wt/Ft: 36

From: 0

To: 805

Cement: $554 \mathrm{Cu}$. Ft.

Note: Hole plugged to surface.

All depths are measured from Kelly Bushing 13' above ground level.

\section{PLUGGING SCHEDULE:}

10/01/77 - Made trip with 7 7/8" bit and conditioned hole. Ran 2 3/8" O.D. tubing in the hole and plugged back hole using Dowell with 10 barrels of water ahead of 48 barrels of mud wash followed by $414 \mathrm{cu}$. $\mathrm{ft}$. of class "C" cement with $2 \%$ calcuim chloride.

Displace cement with 12.5 barrels of water. Cement in place at 1300 hours. Pulled tubing and waited on cement.

10/03/77 - Made trip with 7 7/8" bit and tagged cement at 3556', conditioned hole. Made up $713 / 16^{\prime \prime}$ core bit and cut core \#32 from $3556^{\prime}$ to $3595^{\prime}$, recovered $10^{\prime}$ of cement. Cut core $\# 33$ from $3595^{\prime}$ to $3623^{\prime}$, recovered $20^{\prime}$ of cement. Cut core \#34 from $3623^{\prime}$ to $3630^{\circ}$.

10/04/77 - Completed core \#34 from 3630' to 3673', recovered 27' of cement. Ran $27 / 8^{\prime \prime}$ O.D. tubing in the hole to $3673^{\prime}$.

Cemented plug \#2 using Dowell with 10 barrels of water ahead of 84 barrels of mud flush followed by 10 barrels of water ahead of $1040 \mathrm{cu}$. ft. of $70 \%$ class " $\mathrm{C}$ " cement and $30 \%$ Litepoz. Cement in place at 1140 hours. Pulled tubing to 2300 ' and curculated hole. Waited on cement. Laid down drill pipe.

$10 / 05 / 77$ - Waited on cement to 0900 hours. Tagged top of plug \#2 at $2335^{\prime}$ with tubing. Cemented plug \#3 with 10 barrels of water, 60 barrels of mud flush and 10 barrels of water, ahead of $1039 \mathrm{cu}$. ft. of cement slurry. Cement in place at 1420 hours. Pulled tubing to $803^{\prime}$ and circulated out mud flush and cement. Waited on cement.

10/06/77 - Tagged top of plug \#3 at 827. Cemented plug \#4 to surface with 10 barrels of water, 1000 gallons of mud flush and 10 barrels of water ahead of $417 \mathrm{cu}$. ft. of cement slurry. Cement in place at 0050 hours. Rigged down government furnished equipment and released rig at 1600 hours.

10/14/77 - Cement had dropped to 11.5' inside the 9 5/8" O.D. casing. Cemented to surface with 6 sacks of cement. Hole plugged 


\section{STRATIGRAPHIC SUMMARY}

Quaternary

Holocene-Eolian Sand

Mescalero Caliche

Triassic

Gatuna Formation

Permian

Dewey Lake Red Beds

Rustler Formation

Magenta Dolomite Member

Culebra Dolomite Member

Salado Formation

Upper Member

MB 101

MB 102

MB 103

MB 104

MB 105

MB 106

MB 107

MB 108

MB 109

MB 111

MB 112

MB 113

MB 114

MB 115

MB 116

McNutt Postash Zone

Vaca Triste Sands

MB 117

MB 118

MB 119

MB 121

MB 122

Union Anhydrite

MB 123

MB 124

MB 125

MB 126

Lower Member

MB 128

MB 129

MB 130

MB 131

MB 132

MB 133

MB 134

MB 135

MB 136

MB 138
$13.0-17.0$

$17.0-164.0$

164.0-378.0

$378.0-640.0$

$378.0-379.0$

$489.0-517.0$

640.0-2350.0

640.0-1078.0

696.0

743.0

$775.0-783.5$

794.5

807.0

817.0

829.0

852.5

879.5-904.5

960.0

978.0

1018.0

1035.0

1068.0

1076.0

1087.0-1521.5

1087.0-1092.5

1162.0

1189.0

1225.0

1264.0

1279.5

1310.0-1330.0

1380.5-1387.0

1399.5-1407.5

1482.5

1521.5

1521.5-2350.0

1556.0

1590.0

1604.0

1674.5

1704.5

1722.0

1771.5-1781.0

1807.0

1855.0-1864.5

1930.0 
MB 139

MB 140

MB 141

MB 142

MB 143

Castile Formation

Anhydrite IV

Cowden Anhydrite

Anhydrite III

Halite II

Anhydrite II

Halite I

Anhydrite I

Bell Canyon Formation

Lamar Limestone

Ramsey Sandstone

Ford Shale

Old Sandstone
1993.0

2053.0-2062.5

2150.9-2155.4

2220.6-2237.4

2302.6-2313.6

2350.0-3842.9

2350.0-2398.2

2398.2-2424.0

2424.0-3097.2

3097.2-3283.7

3283.7-3377.4

3377.4-3616.4

3616.4-3842.9

$3842.9-4430.0$ (T.D.)

3842.9-3870.0

3870.0-3918.0

3918.0-3928.0

3928.0-3956.0 
BOREHOLE:

OPERATOR:

PERMIT NO.

LOCATION:

ELEVATION:

TOTAL DEPTH:

TYPE OF WELL:

DRILLER:

DRILLING RECORD:

\section{BABY-1}

U.S. Department of Energy

1989.5' FNL, 2017.1' FEL

Sec 5, T 23 S, R 31 E

3328.38' (Top of Casing)

4298.6

Oil and Gas Well (Exploratory)

Michel P. Grace - 1974 and Salazar Bros.- 1983

Date Started: $\quad 08 / 12 / 83$

Date Completed: $02 / 08 / 75$

Deepened to 4298.6

Date Started: $17 / 18 / 83$

Date Completed: $08 / 19 / 83$

CASING RECORD:

$133 / 8^{\prime \prime}$ O.D. casing set in cement to surface $650^{\prime}$

$97 / 8^{\prime \prime}$ O.D. $650-4159^{\prime}$

7 27/32" O.D. 4159 - 4298.6

PLUGGING SCHEDULE:

Ran 20 joints of $133 / 871 \#$ casing set at $650^{\circ}$. Cemented with 275 sacks of Trin. Lt. Wt. and 125 sacks Cl. C. and $300 \# \mathrm{CaCl}$ and 69\# celloflake.

STRATIGRAPHIC SUMMARY: Attached 


\section{STRATIGRAPHIC SUMMARY}

Quaternary

Triassic

Santa Rosa Sandstone

Permian

Dewey Lake Red Beds

Rustler Formation

Magenta Dolomite Member

Culebra Dolomite Member

Salado Formation

Upper Member

MB 101

MB 102

MB 103

MB 105

MB 106

MB 107

MB 108

MB 109

MB 111

MB 112

MB 113

MB 114

MB 115

MB 116

McNutt Potash Zone

MB 117

MB 118

MB 119

MB 120

MB 121

MB 122

Union Anhydrite

MB 123

MB 124

MB 126

Lower Member

MB 127

MB 128

MB 129

MB 130

MB 131

MB 132

MB 133

MB 134

MB 135

MB 136

MB 137

MB 138

MB 139

MB 140
15.5

$$
15.5-142.8
$$

142.8-362.9

362.9-653.0

375.0-414.1

469.0-487.2

$653.0-2703.5$

781.3

818.0

$845.0-851.0$

884.2

922.1

946.8

962.1

977.0-993.9

1036.9

1065.8

1097.0

1117.1

1157.1

1169.8

1182.0-1585.1

1251.0

1277.6

1299.9

1328.9

1346.1

1353.2

1381.8-1395.2

1454.5-1463.1

1470.0-1481.0

1585.1

1585.1-2703.5

1610.2

1621.8

1650.1

1663.2

1732.9

1763.7

1784.2

1837.7-1848.9

1871.9

1871.9

1905.2-1912.0

1930.5

1990.4

2040.7

2104.0-2110.6 
BABY-1

MB 141

2188.8-2196.9

MB 142

2239.8-2259.0

MB 143

2314.1-2321.0

MB 144

2356.0-2370.0

Cowden Anhydrite

2407.7-2434.7

Castile Formation

2703.5-4045.0

Anhydrite III

2703.5-3154.8

Halite II

3154.8-3373.1

Anhydrite II

$3373.1-3480.0$

Halite I

3480.0-3810.0

Anhydrite I

Bell Canyon Formation

$3810.0-4045.0$

Lamar Limestone

4045.0-T.D.

Ramsey Sandstone

4045.0-4086.0

Ford Shale

4086.0-4132.0

Olds Sandstone

4132.0-4140.0

Hays Sandstone

4140.0-4171.0

4171.0-4298.6 
BOREHOLE:

OPERATOR:

PERMIT NO.:

LOCATION:

ELEVATION:

TOTAL DEPTH:

TYPE OF WELL:

DRILLER:

DRILLING RECORD:

CASING RECORD:

Diameter: $41 / 2$

Grade:

$\mathrm{Wt} / \mathrm{Ft}:$

From: 0

To: $528^{\prime}$

Cement: Cement in place

PLUGGING SCHEDULE:

From: $1411^{\prime}$

To: 528

Int:

Material: Concrete Plug

STRATIGRAPHIC SUMMARY: Attached
D-268

U.S. Department of Energy

Unkown

720.4 FSL, 762.7 FEL, Sec.

35 , T 22 S, R 30 E

3280.70 (Top of Casing)

$1411^{\prime}$

Old Potash Drillhole

Pennsylvania Drilling Co.

Date Started: $\quad 11 / 15 / 89$

Date Completed: 


\section{Quaternary}

Holocene-Eolian Sand

Mescalero Caliche

Triassic

Santa Rosa Sandstone

Permian

Dewey Lake Red Beds

Rustler Formation

Megenta Dolomite Member

Culebra Dolomite Member

Salado Formation

Upper Member

MB 101

MB 102

MB 103

MB 104

MB 105

MB 106

MB 107

MB 108

MB 109

MB 111

MB 112

MB 114

MB 116

McNutt Potash Zone

Vaca Triste Sandstone

MB 117

MB 118

MB 119

MB 121

MB 122

Union Anhydrite

MB 123

MB 124

MB 126

Lower Member

MB 128

MB 129

MB 131

MB 132
$0-15.0$

$15.0-30.0$

$30.0-187.0$

$187.0-494.0$

255.0-275.0

369.0-392.0

469.0 
BOREHOLE:

OPERATOR:

PERMIT NO.:

LOCATION:

ELEVATION:

TOTAL DEPTH:

TYPE OF WELL:

DRILLER:

DRILLING RECORD:

\section{CASING RECORD:}

Diameter: $103 / 4$ O.D.

Grade:

$\mathrm{Wt} / \mathrm{Ft}$ :

From: 0

To: 41

Cement: Set and cemented

Diameter: $103 / 4$ O.D.

Grade: $\mathbf{J}-55$

$\mathrm{Wt} / \mathrm{Ft}: 40.5$

From: 49

To: 1126.2

Cement:

Diameter: $77 / 8$

Grade:

$\mathrm{Wt} / \mathrm{Ft}$ :

From: 1126.2

To: 4057

Cement: Uncased

PLUGGING SCHEdULE:

No plugging data.

STRATIGRAPHIC SUMMARY: Attached
DOE-1

Westinghouse

Unknown

182.4' FSL, 607.8' FEL

Sec. 28, T 22 S, R 31 E

3465.22' (Top of Casing)

4057.3'

Geologic Exploration/Hydrologic Test Hole Salazar Bros. Drilling Co.

Date Started: $\quad 07 / 14 / 82$

Date Completed: $07 / 28 / 82$ 
Quaternary

Eolian Sand

Gatuna Formation

Triassic

Santa Rosa Sandstone

Permian

Dewey Lake Red Beds

Rustler Formation

Magenta Dolomite Member

Culebra Dolomite Member

Salado Formation

Upper Member

MB 101

MB 102

MB 103

MB 105

MB 106

MB 107

MB 108

MB 109

MB 111

MB 112

MB 113

MB 114

MB 115

MB 116

McNutt Potash Zone

Vaca Triste Sandstone

MB 117

MB 118

MB 119

MB 120

MB 121

MB 122

Union Anhydrite

MB 123

MB 124

MB 126

Lower Member

MB 127

MB 128

MB 129

MB 130

MB 131

MB 132

MB 133

MB 134

MB 135

MB 136

MB 137
Not Described

Not Described

$46.0-133.0$

133.0-667.5

667.5-976.5

$722.0-745.0$

828.6-850.5

976.5-2936.5

976.5-1486.0

1102.0

1138.5

1159.0-1169.0

1199.0

1216.0

1254.0

1263.0

1286.0-1309.5

1361.3

1379.8

1406.9

1429.3

1465.8

1477.6

1486.0-1880.3

1486.0-1489.8

1557.2

1582.6

1608.0

1632.0

1646.5

1656.0

1681.4-1694.0

1762.0-1769.9

1773.0-1783.8

1880.3

1880.3-2936.5

1907.4

1919.7

1944.3

1956.7

2025.8

2056.6

2076.0

2117.0-2130.0

2149.4

2192.1-2197.1

2209.2 
STRATIGRAPHIC SUMMARY

DOE-1

MB 138

2264.0

MB 139

2323.7

MB 140

2374.5-2389.0

MB 141

2457.0

MB 142

2497.0-2512.0

MB 143

2563.5-2570.5

MB 144

2606.0-2621.5

Cowden Anhydrite

2647.8-2677.0

Castile Formation

2936.5 (T.D.)

Anhydrite III

2936.5-3374.8

Halite II

3374.8-3600.0

Anhydrite II

3600.0-3708.3

Halite I

3708.3-4032.3

Anhydrite I

4032.3-(T.D.) 
BOREHOLE:

OPERATOR:

PERMIT NO.:

LOCATION:

ELEVATION:

TOTAL DEPTH:

TYPE OF WELL:

DRILLER:

DRILLING RECORD:
DOE-2

U.S. Department of Energy

0.08.1467(State Engineer's Office)

704.07' FSL, 128.19' FEL

Sec. 8, T 22 S, R 31 E

3419.09' (Top of Casing)

$4325^{\prime}$

Geologic Exploration/Hydrologic Test Hole

Unkown

Date Started: $\quad 09 / 08 / 84$
Date Completed: $09 / 18 / 84$

\section{CASING RECORD:}

Diameter: 9.625

Grade: J-55

Wt/Ft: 36

From: 0

To: 1009

Cement:

Diameter: $133 / 8$ O.D.

Grade: $\mathrm{H}-40$

$\mathrm{Wt} / \mathrm{Ft}: 48 \#$

From: 0

To: 39

Cement: Cemented with $81 \mathrm{Cu}$. Ft.

PLUGGING SCHEDULE:

No plugging data.

STRATIGRAPHIC SUMMARY: Attached 


\section{STRATIGRAPHIC SUMMARY}

Quaternary

Holocene-Dune Sand

0-8

Pleistocene-Mescalero Caliche

Triassic

Santa Rosa Sandstone

Permian

Dewey Lake Red Beds

Rustler Formation

Forty-Niner Member

Magenta Dolomite Member

Tamarisk Member

Culebra Dolomite Member

Unnamed Lower Member

Salado Formation

Upper Member

MB 101

MB 102

MB 103

MB 104

MB 105

MB 106

MB 107

MB 108

MB 109

MB 110

MB 111

MB 112

MB 113

MB 114

MB 115

MB 116

McNutt Potash Zone

Vaca Triste Sandstone

MB 117

MB 118

MB 119

10th Ore Zone

MB 120

9th Ore Zone

MB 121

MB 122

8th Ore Zone

Union Anhydrite

MB 123

MB 124

4th Ore Zone

3rd Ore Zone

2nd Ore Zone

MB 125

MB 126
8-13

13-133.3

133.3-639.1

639.1-698.6

698.6-722.4

722.4-823.7

$823.7-846.0$

846.0-960.9

960.9-3082.8

960.9-1448.7

1080.3-1084.2

1116.6-1117.7

1130.4-1143.5

1154.7-1155.1

1170.8-1171.8

Not Present

1228.0-1228.4

1237.5-1238.1

1260.0-1283.5

Not Present

1330.8-1331.0

1347.0-1349.2

1372.4-1372.9

1394.3-1394.8

1427.7-1430.6

1439.3-1441.4

1448.7-1827.4

1448.7-1456.1

1510.0-1511.9

1533.6-1534.7

1556.8-1557.9

1574.0-1580.0

1581.4-1581.8

1580-1584(EST.)

1598.5-1599.8

1606.6-1607.5

1611.1-1619.3

1630.1-1637.9

1716.5-1721.9

1728.8-1738.4

1746.0-1748.5

1766-1774(EST.)

1780-1782.5(EST.)

Not Present

1825.9-1827.4 


\section{STRATIGRAPHIC SUMMARY}

DOE-2

Lower Member
MB 127
MB 128
MB 129
MB 130
MB 131
MB 132
MB 133
MB 134
MB 135
MB 136
MB 137
MB 138
MB 139
MB 140
MB 141
MB 142
MB 143
MB 144

Cowden Anhydrite

Castile Formation

Anhydrite III

Halite II

Anhydrite II

Halite I

Anhydrite I

Delaware Mountain Group

Bell Canyon Formation

Lamar Limestone Member

Ramsey Sand

Ford Shale

Olds Sand

Hays Sand
1827.4-3082.8

1852.7-1853.8

1864.5-1865.5

1889.9-1891.9

1901.8-1902.0

1971.2-1971.7

1999.7-2001.2

2018.8-2021.7

2069.3-2081.0

2099.8-2100.5

2144.9-2157.3

Not Present

2203.1

2303.3-2306.3

2372.1-2388.0

2450.1-2454.5

2503.6-2517.9

2566.4-2571.6

2603.6-2615.7

2644.5-2669.5

3082.8-4071.4

3082.8-3801.1

Not Present

?

3801.1-3809.2

$3809.2-4071.4$

4071.4-4325+

4071.4-4103.4

4103.4-4174.0

4174.0-4182.8

4182.8-4218.2(?)

4218.2(?)-4248+ 


\section{GEOLOGIC EXPLORATION BOREHOLE DATA BASE}

\section{BOREHOLE: \\ OPERATOR: \\ PERMIT NO.:}

LOCATION:

ELEVATION:

TOTAL DEPTH:

TYPE OF WELL:

DRILLER:

DRILLING RECORD:

CASING RECORD:
WIPP-11

Sandia National Laboratories

0.08.994 (State Engineer's Office)

711.80' FNL, 294.08' FWL

Sec. 9, T 22 S, R 31 E

$3426.07^{\prime}$

$3580^{\circ}$

Geologic Exploration

Verna Drilling Compa

Date Started: $\quad 02 / 06 / 78$

Date Completed: $\quad 03 / 14 / 78$

Diameter: $133 / 8$

Grade: $\mathrm{H}-40$

$\mathrm{Wt} / \mathrm{Ft}: 48$

From: 0

To: 40

Cement: $81 \mathrm{Cu} . \mathrm{Ft}$.

Diameter: $95 / 8$

Grade: J-55

Wt/Ft: 36

From: 0

To: 985

Cement: $656 \mathrm{Cu} . \mathrm{Ft}$.

Note: Hole loaded with brine based mud, hole temporarily capped pending further testing and/or plugging, all depths are measured from Kelly Bushing $13 \mathrm{ft}$. above ground level.

No plugging data.

STRATIGRAPHIC SUMMARY: Attached 


\section{STRATIGRAPHIC SUMMARY}

BOREHOLE

WIPP-11
ROCK UNIT

MB 136-T

MB 136-B

MB 138-T

MB 138-B

Anhydrite A

Anhydrite B

MB 139-T

MB 139-B

MB 140-T

MB 140-B
DEPTH INTERVAL IN FEET

1978.5

1978.5-1986.0

$1986-2025.0$

2025-2027

Not Encountered

Not Encountered 2061.0

2061-2064

2064-2092

2092-2105 


\section{GEOLOGIC EXPLORATION BOREHOLE DATA BASE}

BOREHOLE:

PERMIT NO.:

LOCATION:

ELEVATION:

TOTAL DEPTH:

TYPE OF WELL:

DRILLER:

DRILLING RECORD:

CASING RECORD:

PLUGGING SCHEDULE:

STRATIGRAPHIC SUMMARY:
WIPP-12

Westinghouse

Unknown

149.4' FSL, 80.4' FEL

Sec. 17, T 22 S, R 31 E

3472.06 (Top of Casing)

3927.5

Geologic Exploration

Pennsylvania Drilling Company

Date Started: $\quad 11 / 17 / 81$

Date Completed: $\quad 12 / 07 / 78$

Diameter: $95 / 8$

Grade: H-40

Wt/Ft: 32.3

From: 39

To: 1001.8

Cement: $475 \mathrm{Cu} . \mathrm{Ft}$.

Diameter: $95 / 8$

Grade: J-55

Wt/Ft: 36

From: 0

To: 39

Cement: $475 \mathrm{Cu} . \mathrm{Ft}$.

Diameter: $95 / 8$

Grade: $\mathrm{H}-40$

Wt/Ft: 32

From: 39

To: 1013

Cement: $475 \mathrm{Cu}$. Ft.

Note: Hole loaded with brine based mud pending further tests. All depths are measured from Kelly Bushing $12.2 \mathrm{ft}$. above ground level.

No plugging data.

Attached 


\section{STRATIGRAPHIC SUMMARY}

Quaternary Deposits

Sand (Holocene-eolian)

Mescalero Caliche

Gatuna Formation

Triassic Rocks

Santa Rosa Sandstone

Permian Rocks

Dewey Lake Red Beds

Rustler Formation

Magenta Dolomite Member

Culebra Dolomite Member

Salado Formation

Upper Member

MB 101

MB 102

MB 103

MB 104

MB 105

MB 106

MB 107

MB 108

MB 109

MB 111

MB 112

MB 113

MB 114

MB 115

MB 116

McNutt Member

Vaca Triste Sandstone Member

MB 117

MB 118

MB 119

MB 120

MB 121

MB 122

Union Anhydrite

MB 123

MB 124

MB 126

Lower Member

MB 127

MB 128

MB 129

MB 130

MB 131

MB 132
$0-16.2$

16.2-19.2

19.2-28.8

$28.8-167.0$

167.0-640.0

640.0-966.0

703.9-727.0

822.0-846.8

$966.0-2737.5$

966.0-1444.0

1084.5

1116.0

1130.0-1141.0

1150.0

1167.6

1183.5

1223.5

1232.5

1254.0-1278.0

1324.0

1338.0-1342.0

1367.0

1389.0

1424.5

1436.0

1444.0-1798.0

1444.0-1447.0

1507.5

1531.0

1552.0-1556.5

1575.0

1588.0

1596.0

1617.0-1625.0

1695.0-1700.8

1708.0-1715.4

1798.0

1798.0-2737.5

1825.0

.1834 .0

1856.0

1867.0

1928.0

1957.5 


\section{STRATIGRAPHIC SUMMARY}

BOREHOLE

WIPP-12
ROCK UNIT

MB 133

MB 134

MB 135

MB 136

MB 138

MB 139

MB 140

MB 141

MB 142

MB 143

MB 144

Cowden Anhydrite

Castile Formation

DEEPENED PORTION OF WIPP-12

Castile Formation

Anhydrite III Member

Halite II Member

Anhydrite II Member

Halite I Member

Anhydrite I Member
DEPTH INTERVAL IN FEET

1976.0

2015.0-2025.0

2040.0

2071.6-2083.1

2135.1

2184.9

2226.1-2238.1

2290.0-2296.0

2332.0-2344.0

2381.5-2387.6

2413.5-2423.9

2445.5-2471.0

2337.5-T.D.

2776.0-T.D.

2776.0-3053.9

3053.9-3281.8

3281.8-3391.0

3391.0-3901.6

3901.6-T.D. 


\section{GEOLOGIC EXPLORATION BOREHOLE DATA BASE}

\section{BOREHOLE:}

OPERATOR:

PERMIT NO.:

LOCATION:

ELEVATION:

TOTAL DEPTH:

TYPE OF WELL:

DRILLER:

DRILLING RECORD:

CASING RECORD:
WIPP-13

Sandia National Laboratories

0.09.1182 (State Engineer's Office)

2565.68' FSL, 1730.59' FWL

Sec. 17, T 22 S, R 31 E

2565.68' (Top of Casing)

3856

Geologic Exploration

Pennsylvania Drilling Company

Date Started: $\quad 07 / 26 / 78$

Date Completed: $\quad$ 10/05/79 (Recompletion)

Diameter: $133 / 8$

Grade:

$\mathrm{Wt} / \mathrm{Ft}: 48$

From: 0

To: 35.5

Cement: $108 \mathrm{Cu} . \mathrm{Ft}$.

Diameter: $95 / 8$

Grade:

Wt/Ft: 36

From: 0

To: 1023

Cement: $665 \mathrm{Cu}$. Ft.

Note: Hole loaded with brine based mud pending further test and/or plugging. All depths are measured from Kelly Bushing $12.2 \mathrm{ft}$. above ground except cores between 570 and $878 \mathrm{ft}$. and Schlumberger logs which were measured from ground level.

No plugging data.

STRATIGRAPHIC SUMMARY: Attached 


\section{STRATIGRAPHIC SUMMARY}

BOREHOLE

WIPP-13
ROCK UNIT

rnary Deposits

Triassic Rocks

Santa Rosa Sandstone

Permian Rocks

Dewey Lake Red Beds

Rustler Formation

Dissolution Residue

Magenta Dolomite Member

Dissolution Residue

Culebra Dolomite Member

Dissolution Residue

Top of Highest Salt in Section

Salado Formation

Upper Member

MB 101

MB 102

MB 103

DEEPENED PORTION OF WIPP-13 (Distance below K.B.)

Permian Rocks

Salado Formation

Upper Member

MB 103

MB 104

MB 105

MB 106

MB 107

MB 108

MB 109

MB 111

MB 112

MB 113

MB 114

MB 115

MB 116

McNutt Member

Vaca Triste Sandstone Member

MB 117

MB 118

MB 119

MB 120

MB 121

MB 122

Union Anhydrite

MB 123

MB 124

MB 126

Lower Member
DEPTH INTERVAL IN FEET

$0-13$

$13-66$

66-517

517-846

$543-550$

565-583

$679-686$

703-726

730-735

745

846-1025

846-1025

967

1003

1018

858.0-2971.6

858.0-1356.7

1030.0-1042.3

1069.8

1091.0

1111.0

1128.0

1136.1

1161.0-1185.0

1232.8

1250.0

1277.9

1300.9

1338.3

1350.0

1356.7-1730.4

1356.7-1359.0

1426.5

1451.5

1478.2

1497.0

1513.8

1522.0

1542.0-1550.0

1628.0

1644.7

1730.4

1730.4-2971.6 


\section{STRATIGRAPHIC SUMMARY}

BOREHOLE

WIPP-13
ROCK UNIT

MB 127

MB 128

MB 129

MB 130

MB 131

MB 132

MB 133

MB 134

MB 135

MB 136

MB 137

MB 138

MB 139

MB 140

MB 141

MB 142

MB 143

MB 144

Cowden Anhydrite

Castile Formation

Anhydrite III

Halite II

Anhydrite II

Halite I

Anhydrite I
DEPTH INTERVAL IN FEET

1757.8

1770.0

1794.0

1804.5

1873.6

1902.0

1924.0

1968.9-1980.6

1996.8

2033.0-2048.0

2063.0

2110.2

2168.3

2221.0-2232.9

2294.6

2341.5-2355.0

2409.0-2417.0

2460.4

2493.9-2521.6

2971.6-3861.6+ (T.D.)

2971.6-3518.7

3518.7-3638.0

3638.0-3727.5

3727.5-3821.0

3821.0-3861.6+ (T.D.) 


\section{GEOLOGIC EXPLORATION BOREHOLE DATA BASE}

\section{BOREHOLE:}

OPERATOR:

PERMIT NO.:

LOCATION:

ELEVATION:

TOTAL DEPTH:

TYPE OF WELL:

DRILLER:

DRILLING RECORD:

CASING RECORD:

PLUGGING SCHEDULE:

STRATIGRAPHIC SUMMARY:
WIPP-14

Sandia National Laboratories

0.08.1458 (State Engineer's Office)

98.57' FSL, 2112.08' FEL

Sec. 9, T 22 S, R 31 E

3429' (G.L.)

$1000^{\prime}$

Geologic Exploration

Boyles Brothers Drilling

Date Started: $\quad 05 / 02 / 81$

Date Completed: $\quad 06 / 08 / 81$

Diameter: 7

Grade: J-55

WtFt: 23

From: 0

To: 111

Cement:

Note: Hole loaded with brine based mud and temporarily abandoned pending further tests and/or plugging.

No plugging data.

Attached 


\section{STRATIGRAPHIC SUMMARY}

WIPP-14

Quaternary Rocks

Sand (Holocene-eolian)

$0-15.4$

Triassic Rocks

Santa Rosa Sandstone

$15.4-141.0$

Permian Rocks

Dewey Lake Red Beds

141.0-638.7

Rustler Formation

638.7-951

Magenta Dolomite Member

706.5-730.0

Culebra Dolomite Member

$817.2-836.2$

Salado Formation (Upper Member)

951.6 


\section{GEOLOGIC EXPLORATION BOREHOLE DATA BASE}

\section{BOREHOLE:}

OPERATOR:

PERMIT NO.:

LOCATION:

ELEVATION:

TOTAL DEPTH:

TYPE OF WELL:

DRILLER:

DRILLING RECORD:

CASING RECORD:

PLUGGING SCHEDULE:

STRATIGRAPHIC SUMMARY: Attached

$810^{\prime}$

From: 0

To: 13

Cement:

From: 13

To: 592

Cement:

$\mathrm{Wt} / \mathrm{Ft}$ :

To: 810

Cement:

From: 0

To: 555

Cement:
WIPP-15

Sandia National Laboratories

0.13 .00 (State Engineer's Office)

2426' FNL, 1973' FWL

Sec. 18, T 23 S, R $35 \mathrm{E}$

3269.34' (G.L.1)

Geologic Exploration

Boyles Brothers Drilling

Date Started: $\quad 03 / 08 / 78 \quad$ Date Completed: $\quad 04 / 04 / 78$

Diameter: 7

Grade: J-55

$\mathrm{Wt} / \mathrm{Ft}: 20$

Diameter: $61 / 8$

Grade: HW

$\mathrm{Wt} / \mathrm{Ft}: 11.3$

Diameter: 4

Grade: Core

From: 592

Diameter: $41 / 2$

Grade: HW

$\mathrm{Wt} / \mathrm{Ft}: 11.3$

Note: Hole loaded with mud and temporarily capped pending further testing and/or plugging. Hole was relinquished to land owner for use as water well to relieve liability for plugging.

No plugging data. 


\section{STRATIGRAPHIC SUMMARY}

BOREHOLE

WIPP-15
ROCK UNIT

Quaternary Deposits

Clay

Marl

Sand

Clay

Sand

Triassic Rocks

Chinle

Santa Rosa Sandstone
DEPTH INTERVAL IN FEET

0-34

34-99

99-153.3

153.3-226

226-547.2

$547.2-790.5$

$790.5-812$ 


\section{GEOLOGIC EXPLORATION BOREHOLE DATA BASE}

\section{BOREHOLE: \\ OPERATOR: \\ PERMIT NO.:}

LOCATION:

ELEVATION:

TOTAL DEPTH:

TYPE OF WELL:

DRILLER:

DRILLING RECORD:

CASING RECORD:
WIPP-16

Sandia National Laboratories

0.08 .1290 (State Engineer's Office) $\quad 0.08 .1182$ (reentry)

2356.6' FSL, 138.8' FWL

Sec. 5, T 21 S, R 30 E

$3383.40^{\prime}$ (Top of Casing)

$1300^{\prime}$

Geologic Exploration

Pennsylvania Drilling Company

Date Started: $\quad 01 / 11 / 80$

Date Completed: $\quad 02 / 08 / 80$

Diameter: $133 / 8$

Grade: $\mathrm{H}-40$

Wt/Ft: 48

From: 0

To: 38

Cement:

Diameter: $41 / 2$

Grade: J-55

$\mathrm{Wt} / \mathrm{Ft}: 10.5$

From: 0

To: 459

Cement:

Note: Hole filled with brine based mud and temporarily abandoned.

No plugging data.
PLUGGING SCHEDULE:

STRATIGRAPHIC SUMMARY: 


\section{GEOLOGIC EXPLORATION BOREHOLE DATA BASE}

BOREHOLE:

OPERATOR:

PERMIT NO.:

LOCATION:

ELEVATION:

TOTAL DEPTH:

TYPE OF WELL:

DRILLER:

DRILLING RECORD:

CASING RECORD:
WIPP-18

Sandia National Laboratories

0.08.1123 (State Engineer's Office)

983.58' FNL, 11.85' FEL

Sec. 20, T 22 S; R 31 E

3458.7' (Top of Casing)

$1060^{\prime}$

Geologic Exploration/Hydrologic Test Hole

Pennsylvania Drilling Company

Date Started: $\quad 03 / 14 / 78$

Date Completed: $\quad 04 / 03 / 78$

Diameter: 7

Grade: Used

Wt/Ft: 20

From: 0

To: 16

Cement:

Note: Hole loaded with mud pending further testing and/or plugging.

No plugging data.

PLUGGING SCHEDULE:

STRATIGRAPHIC SUMMARY: Attached 


\section{STRATIGRAPHIC SUMMARY}

WIPP-18

Quaternary Deposits

Holocene Deposits

$0-5$

Mescalero Caliche

$5-9$

Triassic Rocks

Santa Rosa Sandstone $\quad 9-138$

Permian Rocks

Dewey Lake Red Beds

$138-613$

Rustler Formation

613-928

Dissolution Residue

643-655

Magenta Dolomite Member

$672-696$

Dissolution Residue

$757-769$

Culebra Dolomite Member

787-808

Dissolution Residue

$812-822$

Salt-bearing Interval

$822-928$

Salado Formation

928-1060

Upper Member

928-1060

MB 101

1049

Maximum Depth Recorded

1060 


\section{GEOLOGIC EXPLORATION BOREHOLE DATA BASE}

BOREHOLE:

OPERATOR:

PERMIT NO.:

LOCATION:

ELEVATION:

TOTAL DEPTH:

TYPE OF WELL:

DRILLER:

DRILLING RECORD:

CASING RECORD:

PLUGGING SCHEDULE:

STRATIGRAPHIC SUMMARY: Attached

$1038^{\prime}$

Wt/Ft: 20

From: 0

To: 8

Cement:
WIPP-19

Sandia National Laboratories

0.08.1124 (State Engineer's Office)

2286.5' FNL, 12.7' FEL

Sec. 20, T 22 S, R $31 \mathrm{E}$

3435.14' (Top of Casing)

Geologic Exploration/Hydrologic Test Hole

Boyles Brothers Drilling

Date Started: $\quad 04 / 06 / 78$

Date Completed: $\quad 05 / 08 / 78$

Diameter: 7

Grade: Used

No plugging data. 


\section{STRATIGRAPHIC SUMMARY}

BOREHOLE

WIPP-19
ROCK UNIT

DEPTH INTERVAL IN FEET

Quaternary Deposits

Holocene Deposits

Mescalero Caliche

Triassic Rocks

Santa Rosa Sandstone

Permian Rocks

Dewey Lake Red Beds

Rustler Formation

Dissolution Residue

Magenta Dolomite Member

Dissolution Residue

Culebra Dolomite Member

Dissolution Residue

Salt-bearing Interval

Salado Formation

Upper Member

MB 101

.Maximum Depth Recorded
$0-7$

7-14

$14-96$

96-590

590-895

619-629

647-672

730-756

756-779

781-795

795-895

895-1038.2

895-1038.2

1010-1012

1034 
BOREHOLE:

OPERATOR:

PERMIT NO.:

LOCATION:

ELEVATION:

TOTAL DEPTH:

TYPE OF WELL:

DRILLER:

DRILLING RECORD:

CASING RECORD:

PLUGGING SCHEDULE:

STRATIGRAPHIC SUMMARY:
WIPP-21

Sandia National Laboratories

0.08.1126 (State Engineer's Office)

1450.6' FSL, 11.7' FEL

Sec. 20, T 22 S, R 31 E

3418.96' (Top of Casing)

1045'

Geologic Exploration/Hydrologic Test Hole

Boyles Brothers Drilling

Date Started: $\quad 05 / 24 / 78$

Date Completed: $\quad 05 / 26 / 78$

Diameter: 7

Grade: J-55

Wt/Ft: 23

From: 0

To: 20

Cement:

Note: Hole loaded with brine mud pending further testing and/or plugging.

No plugging data.

Attached 


\section{STRATIGRAPHIC SUMMARY}

Quaternary Deposits

Holocene Deposits

0-6

Mescalero Caliche

6-12

Gatuna Formation

12-39

Triassic Rocks

Santa Rosa Sandstone

39-73

Permian Rocks

Dewey Lake Red Beds

$73-560$

Rustler Formation $560-868$

Dissolution Residue

588-601

Magenta Dolomite Member

618-642

Dissolution Residue

706-715

Culebra Dolomite Member

729-753

Dissolution Residue

755-759

Salt-bearing Interval

$770-868$

Salado Formation

868-1046

Upper Member

868-1046

MB 101

986-989

MB 102

MB 103

1025-1026

1039

Maximum Depth Recorded

1046 


\section{GEOLOGIC EXPLORATION BOREHOLE DATA BASE}

BOREHOLE:

OPERATOR:

PERMIT NO.:

LOCATION:

ELEVATION:

TOTAL DEPTH:

TYPE OF WELL:

DRILLER:

DRILLING RECORD:

CASING RECORD:
WIPP-22

Sandia National Laboratories

0.08.1127 (State Engineer's Office)

2544.9' FSL, 10.82' FEL

Sec. 20, T 22 S, R 31 E

3428.12' (Top of Casing)

$1450^{\prime}$

Geologic Exploration/Hydrologic Test Hole

Boyles Brother Drilling

Date Started: $\quad 05 / 08 / 78$

Date Completed: $\quad 05 / 24 / 78$

Diameter: 7

Grade: Used

Wt/Ft: 20

From: 0

To: 20

Cement:

Note: Hole loaded with mud pending further testing and/or plugging.

No plugging data.

PLUGGING SCHEDULE:

STRATIGRAPHIC SUMMARY: Attached 


\section{STRATIGRAPHIC SUMMARY}

\section{WIPP-22}

Quaternary Deposits

Holocene Deposits

Mescalero Caliche

Triassic Rocks

Santa Rosa Sandstone

Permian

Dewey Lake Red Beds

Rustler Formation

Dissolution Residue

Magenta Dolomite Member

Dissolution Residue

Culebra Dolomite Member

Dissolution Residue

Salt Interval

Salado Formation

Upper Member

MB 101

MB 102

MB 103

MB 104

MB 105

MB 106

MB 107

MB 108

MB 109

MB 111

MB 112

MB 113

MB 114

MB 115

MB 116

McNutt Potash Unit

Vaca Triste Sandstone Member

MB 117

Maximum Depth Recorded
0-6

6-13

13-81

81-574

574-885

603-614

$630-654$

$717-728$

$742-764$

$767-773$

$777-885$

885-1450

883-1363

1000-1003

1036-1037

1049-1063

1071-1072

1086-1087

1102-1103

1142-1143

1150-1151

1172-1196

1242-1243

1259-1261

1285-1286

1307-1308

1342-1344

1353-1355

1363-1450

1363-1367

1426-1427

1448 . 


\section{GEOLOGIC EXPLORATION BOREHOLE DATA BASE}

BOREHOLE:

OPERATOR:

PERMIT NO.:

LOCATION:

ELEVATION:

TOTAL DEPTH:

TYPE OF WELL:

DRILLER:

DRILLING RECORD:

CASING RECORD:

PLUGGING SCHEDULE:

STRATIGRAPHIC SUMMARY:
WIPP-25

Sandia National Laboratories

0.08.1172 (State Engineer's Office)

1852.77' FSL, 2838.10' FEL

Sec. 15, T 22 S, R 30 E

3214.39' (Top of Casing)

$650^{\prime}$

Geologic Exploration/Hydrologic Test Hole

Boyles Brothers Drilling

Date Started: $\quad 08 / 28 / 78 \quad$ Date Completed: $\quad 09 / 12 / 78$

Diameter: 7

Grade: J-55

Wt/Ft: 23

From: 0

To: 21

Cement:

Diameter: $51 / 2$

Grade: K-55

$\mathrm{Wt} / \mathrm{Ft}: 15.5$

From: 0

To: 649

Cement: $269 \mathrm{Cu}$. Ft.

Note: 7" casing pulled. Culebra perforated from $445-475$ with 120 holes spaced at 4 holes per foot. Magenta perforated from 300-330 with 120 holes spaced at 4 holes per foot. Rustler perforated from 579-608 with 116 holes spaced at 4 holes per foot. Top of Pip Packers set at 572.7 and 365.1 .

No plugging data.

Attached 


\section{STRATIGRAPHIC SUMMARY}

Pleistocene Deposits

Permian Rocks

Dewey Lake Red Beds

Rustler Formation

Dissolution Residue

Magenta Dolomite Member

Dissolution Residue

Culebra Dolomite Member

Dissolution Residue

Salado Formation

Upper Member

Dissolution Residue

MB 101

MB 102

Salt Interval

MB 103

MB 104

MB 105

Maximum Depth Recorded on Geophysical Logs
0-17

$17-232$

232-565

270-287

302-328

$415-424$

$447-472$

475-512

565-655 (T.D.)

$565-600$

$565-600$

589

599

600-655

615

628

640

651 


\section{GEOLOGIC EXPLORATION BOREHOLE DATA BASE}

BOREHOLE:

OPERATOR:

PERMIT NO.:

LOCATION:

ELEVATION:

TOTAL DEPTH:

TYPE OF WELL:

DRILLER:

DRILLING RECORD:

CASING RECORD:

PLUGGING SCHEDULE:

STRATIGRAPHIC SUMMARY:
WIPP-26

Sandia National Laboratories

0.08.1173 (State Engineer's Office)

2232.27' FNL, 12.20' FEL

Sec. 29, T 22 S, R 30 E

3153.20' (Top of Casing)

$503^{\prime}$

Geologic Exploration/Hydrologic Test Hole

Pennsylvania Drilling Company

Date Started: $\quad 08 / 28 / 78 \quad$ Date Completed: $\quad 09 / 11 / 78$

Diameter: 7

Grade: J-55

$\mathrm{Wt} / \mathrm{Ft}: 23$

From: 0

To: 268

Cement:

Diameter: $51 / 2$

Grade: J-55

Wt/Ft: 15.5

From: 0

To: 502

Cement: $510 \mathrm{Cu}$. Ft.

Note: 7 " casing pulled. Culebra perforated from $185-210$ with 100 holes spaced at 4 holes per foot. Magenta perforated from 70-100 wih 120 holes spaced at 4 holes per foot, and from 50-70 with 80 holes spaced at 4 holes per foot. Rustler perforated from 288-329 with 164 holes spaced at 4 holes per foot. Top of Pip Packers set at 269 and 139.1.

No plugging data.

Attached 


\section{STRATIGRAPHIC SUMMARY}

WIPP-26

Holocene Deposits

Permian Rocks

Rustler Formation

Dissolution Residue

Magenta Dolomite Member

Dissolution Residue

Culebra Dolomite Member

Dissolution Residue

Salado Formation

Upper Member

Dissolution Residue

Salt Interval

MB 101

MB 102

MB 103

MB 104

MB 105

MB 106

Maximum Depth Recorded
0-10

10-309

34-50

70-99

152-174

186-209

213-234

309-503

309-503

309-320

320-503

387

423

460

469

481

495

503 


\section{GEOLOGIC EXPLORATION BOREHOLE DATA BASE}

BOREHOLE:

OPERATOR:

PERMIT NO.:

LOCATION:

ELEVATION:

TOTAL DEPTH:

TYPE OF WELL:

DRILLER:

DRILLING RECORD:

CASING RECORD:

PLUGGING SCHEDULE:

STRATIGRAPHIC SUMMARY:
WIPP-27

Sandia National Laboratories

0.08.1174 (State Engineer's Office)

89.79' FNL, 1485.03' FWL

Sec. 21, T 21 S, R 30 E

3178.98' (Top of Casing)

$592^{\prime}$

Geologic Exploration/Hydrologic Test Hole

Boyles Brothers Drilling

Date Started: $\quad 09 / 12 / 78$

Date Completed: $\quad 10 / 09 / 78$

Diameter: $51 / 2$

Grade: J-55

$\mathrm{Wt} / \mathrm{Ft}: 15.5$

From: 0

To: 588

Cement: $440 \mathrm{Cu} . \mathrm{Ft}$.

Note: Culebra perforated from 290-320 with 120 holes spaced at 4 holes per foot. Magenta perforated from 175-195 with 80 holes spaced at 4 holes per foot. Rustler perforated from 425-460 with 135 holes and from $483-513$ with 120 holes spaced at 4 holes per foot. Top of Pi Packers set at 399.4 and 267.4 .

No plugging data.

Attached 


\section{STRATIGRAPHIC SUMMARY}




\section{GEOLOGIC EXPLORATION BOREHOLE DATA BASE}

BOREHOLE:

OPERATOR:

PERMIT NO.:

LOCATION:

ELEVATION:

TOTAL DEPTH:

TYPE OF WELL:

DRILLER:

DRILLING RECORD:

CASING RECORD:

PLUGGING SCHEDULE:

STRATIGRAPHIC SUMMARY:
WIPP-28

Sandia National Laboratories

0.08.1175 (State Engineer's Office)

98.72' FNL, 2400.99' FEL

Sec. 18, T 21 S, R $31 \mathrm{E}$

3349.21' (Top of Casing)

$801^{\prime}$

Geologic Exploration/Hydrologic Test Hole

Pennsylvania Drilling Company

Date Started: $\quad 08 / 07 / 78 \quad$ Date Completed: $08 / 28 / 78$

Diameter: $95 / 8$

Grade: $\mathbf{J}-55$

Wt/Ft: 36

From: 0

To: 21

Cement: Ready Mix

Diameter: 7

Grade: J-55

$\mathrm{Wt} / \mathrm{Ft}: 23$

From: 0

To: 223

Cement:

Diameter: $51 / 2$

Grade: J-55

WtFt: 15.5

From: 0

To: 800

Cement: $314 \mathrm{Cu}$. Ft.

Note: 7 " casing pulled. Culebra perforated from $420-446$ with 104 holes spaced at 4 holes per foot. Magenta perforated from 285-310 with 100 holes spaced at 4 holes per foot. Rustler perforated from 549-589 with 160 holes spaced at 4 holes per foot. Top of Pip. Packers set at 526.7 and 365.1 .

No plugging data.

Attached 


\section{STRATIGRAPHIC SUMMARY}

Holocene Deposits

Permian Rocks

Dewey Lake Red Beds

Rustler Formation

Magenta Dolomite Member

Culebra Dolomite Member

Salado Formation

Upper Member

Dissolution Residue

MB 101

MB 103

Salt Interval

MB 105

MB 106

MB 107

MB 108

MB 109

MB 111

MB 112

MB 113

MB 114

Maximum Depth Recorded
$0-12$

$12-215$

215-531

285-310

420-446

531-801 (T.D)

531-801

531-589

567

585

589-801

598

612

656

664

694

731

743

769

791

802 


\section{GEOLOGIC EXPLORATION BOREHOLE DATA BASE}

BOREHOLE:

OPERATOR:

PERMIT NO.:

LOCATION:

ELEVATION:

TOTAL DEPTH:

TYPE OF WELL:

DRILLER:

DRILLING RECORD:

CASING RECORD:

PLUGGING SCHEDULE:

STRATIGRAPHIC SUMMARY:

\section{WIPP-29}

Sandia National Laboratories

0.08.1176 (State Engineer's Office)

406.62' FSL, $1827.54^{\prime}$ FEL

Sec. 34, T 22 S, R 29 E

2978.26' (Top of Casing)

$377^{\prime}$

Geologic Exploration/Hydrologic Test Hole

Pennsylvania Drilling Company

Date Started: $\quad 10 / 03 / 78$

Date Completed: $10 / 10 / 78$

Diameter: $51 / 2$

Grade: $\mathrm{J}-55$

Wt/Ft: 15.5

From: 0

To: 376

Cement: $135 \mathrm{Cu} . \mathrm{Ft}$.

Note: Top joint of 5.5 casing is $14 \mathrm{lb}$. Culebra perforated from $10-45$ with 140 holes spaced at 4 holes per foot. Rustler perforated from $216-250$ with 136 boles spaced at 4 holes per foot. Top of Pip Packers set at 203.7.

No plugging data.

Attached 


\section{STRATIGRAPHIC SUMMARY}

Holocene Deposits

Permian Rocks

Rustler Formation

Culebra Dolomite Member

Salado Formation

Upper Member

Dissolution Residue

MB 101

MB 102

MB 103

MB 109

McNutt Potash Zone

Dissolution Residue

Vaca Triste Sandstone Member

Salt Interval

MB 117

MB 118

Maximum Depth Recorded
0-12

12-143

12-42

143-377 (T.D.)

143-248

$143-248$

175

181

199

228

248-377 (T.D.)

248-251

248-251

251-377

319

346

358 


\section{GEOLOGIC EXPLORATION BOREHOLE DATA BASE}

\section{BOREHOLE: \\ OPERATOR: \\ PERMIT NO.:}

LOCATION:

ELEVATION:

TOTAL DEPTH:

TYPE OF WELL:

DRILLER:

DRILLING RECORD:

CASING RECORD:

PLUGGING SCHEDULE:

STRATIGRAPHIC SUMMARY:
WIPP-30

Sandia National Laboratories

0.08.1177 (State Engineer's Office)

667.5' FNL, 177.41' FWL

Sec. 33, T 21 S, R 30 E

3429.05' (Top of Casing)

913'

Geologic Exploration/Hydrologic Test Hole

Pennsylvania Drilling Company

Date Started: $\quad 09 / 08 / 78 \quad$ Date Completed: $10 / 02 / 78$

Diameter: 7

Grade: J-55

$\mathrm{Wt} / \mathrm{Ft}: 23$

From: 0

To: 246

Cement:

Diameter: $51 / 2$

Grade: J-55

$\mathrm{Wt} / \mathrm{Ft}: 15.5$

From: 0

To: 912

Cement: $463 \mathrm{Cu}$. Ft.

Note: 7" casing pulled. Culebra perforated from 631-654 with 92 holes spaced at 4 holes per foot. Magenta perforated from 510-540 with 120 holes spaced at 4 holes per foot. Rustler perforated from 731-753 with 88 holes spaced at 4 holes per foot. Top of Pip Packers set at 701.1 and 585.4.

No plugging data.

Attached 


\section{STRATIGRAPHIC SUMMARY}

\section{WIPP-30}

\section{Permian Rocks \\ Dewey Lake Red Beds \\ Rustler Formation}

Magenta Dolomite Member

Culebra Dolomite Member

Salado Formation

Upper Member

Salt Interval

MB 101

MB 102

MB 103

Maximum Depth Recorded
$0-449$

449-748

513-537

631-653

748-912 (T.D.)

748-912

748-912

862

894

In Bed at Total Depth 908 


\section{GEOLOGIC EXPLORATION BOREHOLE DATA BASE}

BOREHOLE:

OPERATOR:

PERMIT NO.:

LOCATION:

ELEVATION:

TOTAL DEPTH:

TYPE OF WELL:

DRILLER:

DRILLING RECORD:

CASING RECORD:
WIPP-31

Sandia National Laboratories

Unknown

422.54' FSL, 1762.24' FWL

Sec. 35, T 20 S, R 30 E

3401.43' (Top of Casing)

$1981.7^{\prime}$

Geologic Exploration

Chortes Drilling Company

Date Started: $\quad 07 / 18 / 80$

Date Completed: $\quad$ 09/29/80 (Recompletion)

Diameter:

Grade: 6' x 6' CEL

$\mathrm{Wt} / \mathrm{Ft}$ :

From: 0

To: 5

Cement: Dirt

Diameter: $95 / 8$

Grade: J-55

Wt/Ft: 36

From: 3

To: 37

Cement: $81 \mathrm{Cu} . \mathrm{Ft}$.

Diameter: $75 / 8$

Grade: $\mathrm{J}-55$

WtFt: 26.4

From: 2.5

To: 808

Cement: $414 \mathrm{Cu} . \mathrm{Ft}$.

Note: Hole loaded with brine based mud pending further testing and/or plugging.

PLUGGING SCHEDULE: No plugging data.

STRATIGRAPHIC SUMMARY: Attached 


\section{STRATIGRAPHIC SUMMARY}

WIPP-31

Quaternary Deposits

Triassic Rocks

Santa Rosa Sandstone

Permian Rocks

Dewey Lake Red Beds

Rustler Formation

Dissolution Residue

Magenta Dolomite Member

Dissolution Residue

Culebra Dolomite Member

Dissolution Residue

Top of Highest Salt in Section

Salado Formation

Upper Member

MB 101

MB 102

MB 103 


\section{GEOLOGIC EXPLORATION BOREHOLE DATA BASE}

\section{BOREHOLE:}

OPERATOR:

PERMIT NO.:

LOCATION:

ELEVATION:

TOTAL DEPTH:

TYPE OF WELL:

DRILLER:

DRILLING RECORD:

CASING RECORD:

PLUGGING SCHEDULE:

STRATIGRAPHIC SUMMARY:
WIPP-32

Sandia National Laboratories

SEO: 04/25/79; USGS: 10/15/79

1673.22' FSL, 29.14' FEL

Sec. 33, T 22 S, R 29 E

3023.26' (Top of Casing)

$390^{\circ}$

Geologic Exploration

Pennsylvania Drilling Company

Date Started: $\quad 08 / 07 / 79$

Date Completed: $\quad 08 / 23 / 79$

No casing used.

Note: Hole plugged to surface with $500 \mathrm{Cu}$. Ft. cement.

Attached 


\section{STRATIGRAPHIC SUMMARY}

WIPP-32

Quaternary Deposits

Triassic Rocks

Santa Rosa Sandstone

Permian Rocks

Dewey Lake Red Beds

Rustler Formation

Dissolution Residue

Magenta Dolomite Member

Dissolution Residue

Culebra Dolomite Member

Dissolution Residue

Top of Highest Salt in Section

Salado Formation

Upper Member

MB 101

MB 102

MB 103 


\section{GEOLOGIC EXPLORATION BOREHOLE DATA BASE}

BOREHOLE:

OPERATOR:

PERMIT NO.:

LOCATION:

ELEVATION:

TOTAL DEPTH:

TYPE OF WELL:

DRILLER:

DRILLING RECORD:

CASING RECORD:
WIPP-33

Sandia National Laboratories

SEO: $04 / 25 / 79$

1762.48' FSL, 2426.65' FWL

Sec. 13, T 22 S, R 30 E

3323.23' (Top of Casing)

$840^{\prime}$

Geologic Exploration

Pennsylvania Drilling Company

Date Started: $\quad 07 / 13 / 79$

Date Completed: 07/26/79

Diameter: $95 / 8$

Grade: J-55

Wt/Ft: 36

From: 0

To: 38

Cement: $192 \mathrm{Cu} . \mathrm{Ft}$.

Note: Hole filled with brine based mud. Fluid level at $27 \mathrm{ft}$.

No plugging data.

STRATIGRAPHIC SUMMARY: Attached 


\section{STRATIGRAPHIC SUMMARY}

WIPP-33

Quaternary Deposits

Triassic Rocks

Santa Rosa Sandstone

Permian Rocks

Dewey Lake Red Beds

Rustler Formation

Dissolution Residue

Magenta Dolomite Member

Dissolution Residue

Culebra Dolomite Member

Dissolution Residue

Top of Highest Salt in Section

Salado Formation

Upper Member

MB 101

MB 102

MB 103 
BOREHOLE:

OPERATOR:

PERMIT NO.:

LOCATION:

ELEVATION:

TOTAL DEPTH:

TYPE OF WELL:

DRILLER:

DRILLING RECORD:

CASING RECORD:
WIPP-34

Sandia National Laboratories

08.08.1291 (State Engineer's Office) 08.08 .1195 (reentry)

201.78' FSL, 1999.73' FWL

Sec. 9, T 22 S, R $31 \mathrm{E}$

$3433^{\prime}$ (Top of Casing)

$1820^{\prime}$

Geologic Exploration

Pennsylvania Drilling Company

Date Started: $\quad 08 / 16 / 79$

Date Completed: $\quad 09 / 04 / 79$

Diameter: $133 / 8$

Grade:

$\mathrm{Wt} / \mathrm{Ft}: 48$

From: 0

To: 38

Cement:

Note: Hole filled with brine based mud.

PLUGGING SCHEDULE:

No plugging data.

STRATIGRAPHIC SUMMARY: Attached 


\section{STRATIGRAPHIC SUMMARY}

Quaternary Deposits .

Triassic Rocks

Santa Rosa Sandstone

Permian Rocks

Dewey Lake Red Beds

Rustler Formation

Magenta Dolomite Member

Culebra Dolomite Member

Salado Formation

Upper Unit

MB 101

MB 102

MB 103

MB 104

MB 105

MB 106

MB 107

MB 108

MB 109

MB 110

MB 111

MB 112

MB 113

MB 114

MB 115

MB 116

McNutt Potash Unit

Vaca Triste Sandstone Member

MB 117

MB 118

MB 119

MB 120

MB 121

MB 122

Union Anhydrite

MB 123

MB 124

MB 125

MB 126

Lower Unit

MB 127

MB 128

MB 129
0-11

$11-154$

154-657

657-973

716-741

834-861

973-1820+

973-1437

1092

1122

1148

1158

1173

1191

1228

1237

1280

1317

1326

1344

1366

1396

1420

1430

1437-1751

1437-1442

1498

1520

1540

1560

1573

1580

1599-1607

1676

1690

1751

1751-1820+

1768

1775

1785 


\section{HYDROLOGIC TEST BOREHOLE DATA BASE}

BOREHOLE:

OPERATOR:

PERMIT NO.:

LOCATION:

ELEVATION:

TOTAL DEPTH:

TYPE OF WELL:

DRILLER:

DRILLING RECORD:

CASING RECORD:

PLUGGING SCHEDULE:

STRATIGRAPHIC SUMMARY:
H-1

Sandia National Labs

0.08.979 (State Engineer's Office)

623.2' FNL, 1083.1' FWL Sec.

29, T 22 S, R 31 E

3399.53' (Top of Casing)

$856^{\prime}$

Hydrologic Test Hole

Pennsylvania Drilling Company

Date Started: $\quad 05 / 20 / 76$

Date Completed: $\quad 06 / 10 / 76$

Diameter: $101 / 8$

Grade:

Wt/Ft: 40.5

From: 0

To: 48

Cement: $51 \mathrm{Cu} . \mathrm{Ft}$.

Diameter: 7

Grade: K-55

$\mathrm{Wt} / \mathrm{Ft}: 26$

From: 0

To: 848

Cement: $192 \mathrm{Cu} . \mathrm{Ft}$.

Note: Perforated from 803 to 827 with 72 holes, 703 to 683 with 3 holes per foot, 683 to 675 with 3 holes per foot, 562 to 590 with 3 holes per foot. All depths are measured from Kelly Bushing $8^{\prime}$ above ground level except DST's which are measured from ground level.

No plugging data.

Attached 


\section{STRATIGRAPHIC SUMMARY}

H-1

\section{Holocene}

Surficial Deposits

$0-15$

Pleistocene

Gatuna Formation

$15-35$

Ochoan

Dewey Lake Red Beds

35-502

Rustler Formation

$502-824$

Magenta Dolomite Member

563-589

Culebra Dolomite Member

676-699

Top of Salado

824

Total Depth

856 
BOREHOLE:

OPERATOR:

PERMIT NO.:

LOCATION:

ELEVATION:

TOTAL DEPTH:

TYPE OF WELL:

DRILLER:

DRILLING RECORD:

CASING RECORD:

\section{H-2a}

Sandia National Labs

0.08.990 (State Engineer's Office)

726.96' FNL, 1697.64', FWL

Sec. 29, T 22 S, R 31 E

$3378.09^{\prime}$ (Top of Casing)

$672^{\prime}$

Hydrologic Test Hole

Pennsylvania Drilling Company

Date Started: $\quad 02 / 14 / 77$

Diameter: $103 / 4$

Grade:

$\mathrm{Wt} / \mathrm{Ft}:$

From: 0

To: 33

Cement: $54 \mathrm{Cu}$. Ft.

Diameter: $65 / 8$

Grade: J-55

$\mathrm{Wt} / \mathrm{Ft}: 24$

From: 0

To: 511

Cement: $260 \mathrm{Cu} . \mathrm{Ft}$.

PLUGGING SCHEDULE: No plugging data.

STRATIGRAPHIC SUMMARY: Attached 


\section{STRATIGRAPHIC SUMMARY}

H-2a
ROCK UNIT

DEPTH INTERVAL IN FEET

\section{Holocene}

Surficial Deposit

Pleistocene

Gatuna Formation

Ochoan

Dewey Lake Red Beds

Rustler Formation

Forty-Niner Member

Magenta Dolomite Member

Tamarisk Member

Culebra Dolomite Member

Unnamed Part

Total Depth

\author{
0-14 \\ 14-38 \\ 38-457 \\ 457-672 (T.D.) \\ $457-515$ \\ 515-543 \\ 543-623 \\ 623-645 \\ 645-672 (T.D.)
}

672 
BOREHOLE:

OPERATOR:

PERMIT NO.:

LOCATION:

ELEVATION:

TOTAL DEPTH:

TYPE OF WELL:

DRILLER:

DRILLING RECORD:

CASING RECORD:

PLUGGING SCHEDULE:

STRATIGRAPHIC SUMMARY: Attached

H-2b1

$661^{\prime}$

Grade:

$\mathrm{Wt} / \mathrm{Ft}$ :

From: 0

To: 33

From: 0

To: 609
Sandia National Labs

0.08.990 (State Engineer's Office)

695.57' FNL, 1660.57' FWL,

Sec. 29, T 22 S, R $31 \mathrm{E}$

3378.46 (Top of Casing)

Hydrologic Test Hole

Pennsylvania Drilling Company

Date Started: $\quad 02 / 07 / 77$

Date Completed:

$02 / 14 / 77$

Diameter: $103 / 4$

Cement: $54 \mathrm{Cu}$. Ft.

Diameter: $65 / 8$

Grade: J-55

$\mathrm{Wt} / \mathrm{Ft}: 24$

Cement: $282 \mathrm{Cu}$. Ft.

Note: Perforated from $510^{\prime}-538^{\prime}$ with 3 holes per foot.

No plugging data. 


\section{STRATIGRAPHIC SUMMARY}

BOREHOLE

H-2b1
ROCK UNIT

DEPTH INTERVAL IN FEET

Holocene

Surficial Deposit

$0-14$

Pleistocene

Gatuna Formation

$14-38$

Ochoan

Dewey Lake Red Beds

Rustler Formation

Forty-Niner Member

Magenta Dolomite Member

Tamarisk Member

Culebra Dolomite Member

Unnamed Part

Total Depth
$38-457$

$457-661$

457-515

$515-543$

543-623

623-645

645-661

661 
BOREHOLE:

OPERATOR:

PERMIT NO.:

LOCATION:

ELEVATION:

TOTAL DEPTH:

TYPE OF WELL:

DRILLER:

DRILLING RECORD:

CASING RECORD:
H-2b2

Sandia National Labs

0.08.990 (State Engineer's Office)

700.6' FNL, 1690.8' FWL, Sec.

29, T 22 S, R 31 E

3378.31' (Top of Casing)

$660^{\prime}$

Hydrologic Test Hole

Pennsylvania Drilling Company

Date Started: $\quad 07 / 16 / 83$

Date Completed: $\quad 05 / 03 / 84$

Diameter: 9.625 0.D.

Grade: J-55

Wt/Ft: 36

From: 0

To: 20

Cement:

Diameter: 5.5 O.D.

Grade: J-55

$\mathrm{Wt} / \mathrm{Ft}: 15.5$

From: 0

To: 620

Cement:

Note: Open 613-650'.

PLUGGING SCHEDULE: No plugging data.

STRATIGRAPHIC SUMMARY: Attached 
STRATIGRAPHIC SUMMARY

$\mathbf{H}-\mathbf{2 b 2}$

Holocene

Surficial Deposits

$0-14$

Quaternary

Gatuna Formation

$14-38$

Permian

Dewey Lake Red Beds

Rustler Formation

$38-457$

Forty-Niner Member

457-660 (T.D.)

Magenta Dolomite Member

$457-515$

Tamarisk Member

515-543

Culebra Dolomite Member

543-623

Unnamed Part

623-645

645-660 (T.D.) 
BOREHOLE:

OPERATOR:

PERMIT NO.:

LOCATION:

ELEVATION:

TOTAL DEPTH:

TYPE OF WELL:

DRILLER:

DRILLING RECORD:

CASING RECORD:
H-2c

Sandia National Labs

0.08.990 (State Engineer's Office)

637.15' FNL, 1708.62' FWL,

Sec. 29, T 22 S, R $31 \mathrm{E}$

3378.41' (Top of Casing)

795

Hydrologic Test Hole

Pennsylvania Drilling Company

Date Started: $\quad 01 / 28 / 77$

Date Completed: $\quad 02 / 05 / 77$

Diameter: $103 / 4$

Grade:

$\mathrm{Wt} / \mathrm{Ft}$ :

From: 0

To: 33

Cement: $54 \mathrm{Cu} . \mathrm{Ft}$.

Diameter: $65 / 8$

Grade: J-55

WtFt: 24

From: 0

To: 742

Cement: $339 \mathrm{Cu}$. Ft.

Note: Perforated from $618-655$ with 3 holes per foot.

PLUGGING SCHEDULE:

No plugging data.

STRATIGRAPHIC SUMMARY:

Attached 


\section{STRATIGRAPHIC SUMMARY}

H-2c

\section{Holocene}

Surficial Deposits

$0-34$

Quaternary

Gatuna Formation

$0-34$

Permian

Dewey Lake Red Beds

$34-457$

Rustler Formation Magenta Dolomite Member

515-540

Culebra Dolomite Member

624-642

Top of Rustler Salt

642

Salado Formation

Maximum Depth

764-795 (T.D.)

795 
BOREHOLE:

OPERATOR:

PERMIT NO.:

LOCATION:

ELEVATION:

TOTAL DEPTH:

TYPE OF WELL:

DRILLER:

DRILLING RECORD:

CASING RECORD:

\section{H-3b1}

Sandia National Lab

0.08.991 (State Engineer's Office)

2085.31' FSL, 138.10' FEL,

Sec. 29, T 22 S, R 31 E

$3390.64^{\prime}$ (Top of Casing)

902 '

Hydrologic Test Hole

Pennsylvania Drilling Company

Date Started: $\quad 07 / 25 / 76$

Date Completed: $\quad 08 / 12 / 76$

Diameter: $103 / 4$

Grade:

$\mathrm{Wt} / \mathrm{Ft}: 40.5$

From: 0

To: 38

Cement: $54 \mathrm{Cu} . \mathrm{Ft}$.

Diameter: $65 / 8$

Grade: J-55

Wt/Ft: 24

From: 0

To: 891

Cement: 625 Sacks

Note: Perforated from 813 to 837 with 72 holes, 683 to 703 with 3 holes per ft, 675 to 683 with 3 holes per $\mathrm{ft}, 562$ to 590 with 3 holes per $\mathrm{ft}$.

No plugging data.

PLUGGing SCHEDULE:

Attached 


\section{STRATIGRAPHIC SUMMARY}

H-3b1

\section{Holocene}

Surficial Deposits

$0-4$

Quaternary

Gatuna Formation

4-22

Permian

Dewey Lake Red Beds

22-502

Rustler Formation

502-821

Magenta Dolomite Member

559-584

Culebra Dolomite Member

672-694

Top of Rustler Salt

Not Given

Salado Formation

Maximum Depth

821- T.D.

902 
BOREHOLE:

OPERATOR:

PERMIT NO.:

LOCATION:

ELEVATION:

TOTAL DEPTH:

TYPE OF WELL:

DRILLER:

DRILLING RECORD:

CASING RECORD:

PLUGGING SCHEDULE:

STRATIGRAPHIC SUMMARY:
H-3b2

Sandia National Labs

0.08.991 (State Engineer's Office)

2122.15' FSL, 231.29' FEL,

Sec. 29, T 22 S, R 31 E

$3390.03^{\prime}$ (Top of Casing)

$725^{\prime}$

Hydrologic Test Hole

Pennsylvania Drilling Company

Date Started:

Date Completed:

Diameter: 5 1/2 O.D.

Grade: J-55

Wt/Ft: 15.5

From: 0

To: 672.7

Cement:

No plugging data.

Attached 


\title{
STRATIGRAPHIC SUMMARY
}

H-3b2

\author{
Surficial Deposits \\ Gatuna Formation \\ Dockum Group \\ Dewey Lake Red Beds \\ Rustler Formation \\ Magenta Dolomite Member \\ Culebra Dolomite Member \\ Salado Formation \\ Upper Member \\ McNutt Member \\ Lower Member \\ Total Depth
}

0-11

$11-65$

$65-565.7$

$565.7-788.7$

564-590

676-700

725 
BOREHOLE:

OPERATOR:

PERMIT NO.:

LOCATION:

ELEVATION:

TOTAL DEPTH:

TYPE OF WELL:

DRILLER:

DRILLING RECORD:

CASING RECORD:

PLUGGING SCHEDULE:

STRATIGRAPHIC SUMMARY:

\section{$\mathbf{H}-\mathbf{3 b 3}$}

Sandia National Labs

0.08.991 (State Engineer's Office)

2022.35' FSL, 217.30' FEL,

Sec. 29, T 22 S, R 31 E

3388.67' (Top of Casing)

$730^{\circ}$

Hydrologic Test Hole

Pennsylvania Drilling Company

Date Started:

Date Completed: $\quad 01 / 30 / 84$

Diameter: 5.5

Grade: J-55

Wt/Ft: 15.5

From: .50

To: 670.5

Cement: Casing cemented in place

No plugging data.

Attached 


\section{STRATIGRAPHIC SUMMARY}

BOREHOLE

H-3b3
Surficial Deposits
Gatuna Formation
Dockum Group
Dewey Lake Red Beds
Rustler Formation

Magenta Dolomite Member

Culebra Dolomite Member

563-586

673-696

Salado Formation

Upper Member

McNutt Member

Lower Member

Castile Formation 


\section{HYDROLOGIC TEST BOREHOLE DATA BASE}

BOREHOLE:

OPERATOR:

PERMIT NO.:

LOCATION:

ELEVATION:

TOTAL DEPTH:

TYPE OF WELL:

DRILLER:

DRILLING RECORD:

CASING RECORD:
H-3d (H-3b4)

Sandia National Labs

0.08.991 (State Engineer's Office)

2067.3' FSL, 164.3' FEL, Sec.

29, T 22 S, R $31 \mathrm{E}$

3390.01' (Top of Casing)

$554^{\circ}$

Hydrologic Test Hole

Pennsylvania Drilling Company

Date Started:

Date Completed:

Diameter: $85 / 8$

Grade:

$\mathrm{Wt} / \mathrm{Ft}$ :

From: 0

To: 39

Cement:

Note: $77 / 8^{\prime \prime}$ uncased borehole 33-559'.

No plugging data.

STRATIGRAPHIC SUMMARY: Attached 


\section{STRATIGRAPHIC SUMMARY}

H-3d

Surficial Deposits

Gatuna Formation

Dockum Group

Dewey Lake Red Beds

Rustler Formation

Forty-Niner Member

508-537

Forty-Niner Claystone Member

$537-547$

Salado Formation

Upper Member

McNutt Member

Lower Member

Castile Formation 


\section{HYDROLOGIC TEST BOREHOLE DATA BASE}

\section{BOREHOLE: \\ OPERATOR: \\ PERMIT NO.:}

LOCATION:

ELEVATION:

TOTAL DEPTH:

TYPE OF WELL:

DRILLER:

DRILLING RECORD:

CASING RECORD:

PLUGGING SCHEDULE:

STRATIGRAPHIC SUMMARY:
H-4a

Sandia National Labs

0.08 .1153 (State Engineer's Office)

545.89' FNL, 720.00' FWL,

Sec. 5, T.23 S, R $31 \mathrm{E}$

3333.29' (Top of Casing)

$415^{\circ}$

Hydrologic Test Hole

Pennsylvania Drilling Company

Date Started: $\quad 04 / 30 / 78$

Date Completed: $\quad 05 / 23 / 78$

Diameter: $95 / 8$

Grade: J-55

Wt/Ft: 36

From: 0

To: 32

Cement: $63 \mathrm{Cu}$. Ft.

Diameter: $51 / 2$

Grade: J-55

Wt/Ft: 15.5

From: 0

To: 364

Cement: $173 \mathrm{Cu} . \mathrm{Ft}$.

Note: Hole in standby condition for hydro tracer tests. $41 / 2^{\prime \prime}$ inflatable packer set at $485^{\circ}$ on $11 / 2$ "galvanized pipe. $13 / 8 "$ pump cylinder set at $499^{\prime}$ in the Culebra Dolomite. The hole is dual completion across the Magenta and Culebra Dolomites.

No plugging data.

Attached 


\section{STRATIGRAPHIC SUMMARY}

H-4a

Holocene

Surficial Deposits

0-13

Pleistocene

Gatuna Formation

13-29

Ochoan

Dewey Lake Red Beds

29-315

Rustler Formation

315

Magenta Dolomite Member

$375-400$

Total Depth

415 


\section{HYDROLOGIC TEST BOREHOLE DATA BASE}

BOREHOLE:

OPERATOR:

PERMIT NO.:

LOCATION:

ELEVATION:

TOTAL DEPTH:

TYPE OF WELL:

DRILLER:

DRILLING RECORD:

CASING RECORD:
H-4b

Sandia National Labs

0.08.1154 (State Engineer's Office)

498.47' FNL, 632.54' FWL,

Sec. 5, T 23 S, R 31 E

3333.35 (Top of Casing)

$529^{\prime}$

Hydrologic Test Hole

Pennsylvania Drilling Company

Date Started: $\quad 04 / 30 / 78$

Date Completed: $\quad 05 / 15 / 78$

Diameter: $95 / 8$

Grade: J-55

$\mathrm{Wt} / \mathrm{Ft}: 36$

From: 0

To: 33

Cement: $63 \mathrm{Cu} . \mathrm{Ft}$.

Diameter: $51 / 2$

Grade: J-55

$\mathrm{Wt} / \mathrm{Ft}: 15.5$

From: 0

To: 476

Cement: $269 \mathrm{Cu} . \mathrm{Ft}$.

Note: Hole is in standby condition for hydro tracer tests.

PLUGGING SCHEDULE:

No plugging data.

STRATIGRAPHIC SUMMARY:

Attached 


\section{STRATIGRAPHIC SUMMARY}

H-4b

Surficial Deposits

$0-13$

Gatuna Formation

13-29

Dockum Group

NP

Dewey Lake Red Beds

29-315

Rustler Formation

315-T.D.

Magenta Dolomite Member

$377-402$

Culebra Dolomite Member

498-522

Salado Formation

Upper Member

McNutt Member

Lower Member

Total Depth 


\section{HYDROLOGIC TEST BOREHOLE DATA BASE}

BOREHOLE:

OPERATOR:

PERMIT NO.:

LOCATION:

ELEVATION:

TOTAL DEPTH:

TYPE OF WELL:

DRILLER:

DRILLING RECORD:

CASING RECORD:

PLUGGING SCHEDULE:

STRATIGRAPHIC SUMMARY: Attached

H-4c

$661^{\prime}$

To: 33

From: 0

To: 610
Sandia National Labs

0.08.1152 (State Engineer's Office)

446.36 FNL, 717.89 FWL,

Sec. 5, T 23 S, R $31 \mathrm{E}$

3334.04' (Top of Casing)

Hydrologic Test Hole

Pennsylvania Drilling Company

Date Started: $\quad 04 / 30 / 78$

Date Completed: $\quad 05 / 09 / 78$

Diameter: $95 / 8$

Grade: J-55

Wt/Ft: 36

From: 0

Cement: $63 \mathrm{Cu} . \mathrm{Ft}$.

Diameter: $51 / 2$

Grade: J-55

Wt/Ft: 15.5

Cement: $270 \mathrm{Cu} . \mathrm{Ft}$.

Note: Bridge plug at 530'. Perforated from 494' to 520' with 104 holes spaced at 4 holes per foot. Hole in standby condition for hydro tracer tests.

No plugging data. 


\section{STRATIGRAPHIC SUMMARY}

BOREHOLE

H-4c
ROCK UNIT

Surficial Deposits

Gatuna Formation

Dockum Group

Dewey Lake Red Beds

Rustler Formation

Magenta Dolomite Member

Culebra Dolomite Member

Salado Formation
DEPTH INTERVAL IN FEET

$0-13$

13-29

NP

29-315

315-626

$377-403$

490-516

626-661 
BOREHOLE:

OPERATOR:

PERMIT NO.:

LOCATION:

ELEVATION:

TOTAL DEPTH:

TYPE OF WELL:

DRILLER:

DRILLING RECORD:

CASING RECORD:

\section{H-5a}

Sandia National Labs

0.08.1159 (State Engineer's Office)

1091.98 FNL, 185.03 FEL,

Sec. 15, T 22 S, R $31 \mathrm{E}$

3506.19 (Top of Casing)

824'

Hydrologic Test Hole

Pennsylvania Drilling Company

Date Started: $\quad 05 / 22 / 78$

Date Completed: $\quad 06 / 20 / 78$

Diameter: $95 / 8$

Grade: J-55

Wt/Ft: 36

From: 0

To: 38

Cement: $72 \mathrm{Cu} . \mathrm{Ft}$.

Diameter: $51 / 2$

Grade: J-55

$\mathrm{Wt} / \mathrm{Ft}: 15.5$

From: 0

To: 774

Cement: $192 \mathrm{Cu} . \mathrm{Ft}$.

Note: Hole in standby condition for hydro tracer tests. $41 / 2^{\prime \prime}$ inflatable packer set at $895^{\prime}$ on $11 / 2$ " galvanized pipe. $13 / 8$ " pump cylinder set at $905^{\prime}$ in the Culebra Dolomite. The hole is dual-completion across the Magenta and Culebra Dolomites.

No plugging data.

PLUGGING SCHEDULE:

STRATIGRAPHIC SUMMARY: 


\section{HYDROLOGIC TEST BOREHOLE DATA BASE}

BOREHOLE:

OPERATOR:

PERMIT NO.:

LOCATION:

ELEVATION:

TOTAL DEPTH:

TYPE OF WELL:

DRILLER:

DRILLING RECORD:

CASING RECORD:
H-5b

Sandia National Lab

0.08.1160 (State Engineer's Office)

1008.30' FNL, 236.22' FEL,

Sec. 15, T 22 S, R 31 E

3506.04' (Top of Casing)

925'

Hydrologic Test Hole

Pennsylvania Drilling Company

Date Started: $\quad 05 / 22 / 78$

Date Completed: $\quad 06 / 13 / 78$

Diameter: $95 / 8$

Grade: J-55

Wt/Ft: 36

From: 0

To: 38

Cement: $72 \mathrm{Cu}$. Ft.

Diameter: $51 / 2$

Grade: J-55

$\mathrm{Wt} / \mathrm{Ft}: 15.5$

From: 0

To: 881

Cement: $336 \mathrm{Cu}$. Ft.

Note: Hole is in standby condition for hydro tracer tests.

PLUGGING SCHEDULE:

No plugging data.

STRATIGRAPHIC SUMMARY: Attached 


\section{STRATIGRAPHIC SUMMARY}

\section{$\mathbf{H - 5 b}$}

Surficial Deposits

Gatuna Formation

0-8

Dockum Group

NP

Dewey Lake Red Beds

8-225

Rustler Formation 225-732

Magenta Dolomite Member

732-T.D.

Culebra Dolomite Member

$785-805$

$897-920$

Salado Formation

Upper Member

McNutt Member

Lower Member

Castile Formation 
BOREHOLE:

OPERATOR:

PERMIT NO.:

LOCATION:

ELEVATION:

TOTAL DEPTH:

TYPE OF WELL:

DRILLER:

DRILLING RECORD:

CASING RECORD:

PLUGGING SCHEDULE:

STRATIGRAPHIC SUMMARY:
H-5c

Sandia National Labs

0.08.1161 (State Engineer's Office)

1005.55 FNL, 134.95 FEL,

Sec. 15, T 22 S, R 31 E

3506.04' (Top of Casing)

$1076^{\circ}$

Hydrologic Test Hole

Pennsylvania Drilling Company

Date Started: $\quad 05 / 22 / 78$

Date Completed: $\quad 06 / 03 / 78$

Diameter: $95 / 8$

Grade: J-55

Wt/Ft: 36

From: 0

To: 38

Cement: $72 \mathrm{Cu} . \mathrm{Ft}$.

Diameter: $51 / 2$

Grade: J-55

Wt/Ft: 15.5

From: 0

To: 1024

Cement: $416 \mathrm{Cu}$. Ft.

Note: Bridge plug set at $935^{\prime}$. Perforated from $895^{\prime}$ to $925^{\prime}$ with 120 holes spaced at 4 holes per foot. Hole in standby condition for hydro tracer tests.

No plugging data.

Attached 


\section{STRATIGRAPHIC SUMMARY}

BOREHOLE

H-5c
ROCK UNIT

Surficial Deposits

Gatuna Formation

Dockum Group

Dewey Lake Red Beds

Rustler Formation

Magenta Dolomite Member

Culebra Dolomite Member

Salado Formation

Upper Member

McNutt Member

Lower Member

Total Depth
DEPTH INTERVAL IN FEET

0-8

NP

8-225

225-732

$732-1041$

788-812

899-924

1041-T.D.

1076 


\section{HYDROLOGIC TEST BOREHOLE DATA BASE}

BOREHOLE:

OPERATOR:

PERMIT NO.:

LOCATION:

ELEVATION:

TOTAL DEPTH:

TYPE OF WELL:

DRILLER:

DRILLING RECORD:

CASING RECORD:

PLUGGING SCHEDULE:

STRATIGRAPHIC SUMMARY:
H-6a

Sandia National Labs

0.08.1162 (State Engineer's Office)

283.30' FNL, 274.34' FWL,

Sec. 18, T 22 S, R 31 E

3347.83' (Top of Casing)

525

Hydrologic Test Hole

Pennsylvania Drilling Company

Date Started: $\quad 07 / 06 / 78$

Date Completed: $\quad 07 / 11 / 78$

Diameter: $95 / 8$

Grade: J-55

Wt/Ft: 36

From: 0

To: 38

Cement: $72 \mathrm{Cu}$. Ft.

Diameter: $51 / 2$

Grade: $\mathrm{J}-55$

Wt/Ft: 15.5

From: 0

To: 475

Cement: $155 \mathrm{Cu}$. Ft.

Note: Hole in standby condition for hydro tracer tests. $41 / 2^{\prime \prime}$ packer set at $594^{\prime}$ on $11 / 2^{\prime \prime}$ galvanized pipe. $13 / 8^{\prime \prime}$ pump cylinder at $608^{\prime}$ in the Culebra Dolomite. The hole is dual-completion across the Magenta and Culebra Dolomites.

No plugging data. 
BOREHOLE:

OPERATOR:

PERMIT NO.:

LOCATION:

ELEVATION:

TOTAL DEPTH:

TYPE OF WELL:

DRILLER:

DRILLING RECORD:

CASING RECORD:
H-6b

Sandia National Labs

0.08.1163 (State Engineer's Office)

196.34' FNL, 332.96' FWL,

Sec 18, T 22 S, R $31 \mathrm{E}$

3348.25' (Top of Casing)

$640^{\prime}$

Hydrologic Test Hole

Pennsylvania Drilling Company

Date Started: $\quad 06 / 19 / 78$

Date Completed: $\quad 07 / 05 / 78$

Diameter: $95 / 8$

Grade: $\mathrm{J}-55$

$\mathrm{Wt} / \mathrm{Ft}: 36$

From: 0

To: 38

Cement: $72 \mathrm{Cu}$. Ft.

Diameter: $51 / 2$

Grade: $\mathbf{J}-55$

$\mathrm{Wt} / \mathrm{Ft}: 15.5$

From: 0

To: 590

Cement: $210 \mathrm{Cu} . \mathrm{Ft}$.

Note: Hole in standby condition for hydro tracer tests.

PLUGGING SCHEDULE:

No plugging data.

STRATIGRAPHIC SUMMARY:

Attached 


\section{STRATIGRAPHIC SUMMARY}

BOREHOLE

H-6b
ROCK UNIT

Surficial Deposits

Gatuna Formation

Dockum Group

Dewey Lake Red Beds

Rustler Formation

Magenta Dolomite Member

Culebra Dolomite Member

Salado Formation

Upper Member

McNutt Member

Lower Member

Total Depth
DEPTH INTERVAL IN FEET

$0-12$

12-38

NP

38-427

427-T.D.

492-511

604-627

640 
BOREHOLE:

OPERATOR:

PERMIT NO.:

LOCATION:

ELEVATION:

TOTAL DEPTH:

TYPE OF WELL:

DRILLER:

DRILLING RECORD:

CASING RECORD:
H-6c

Sandia National Labs

0.08.1164 (State Engineer's Office)

281.06' FNL, 374.47' FWL,

Sec. $18, T 22$ S, R $31 \mathrm{E}$

3348.52' (Top of Casing)

$741^{\prime}$

Hydrologic Test Hole

Pennsylvania Drilling Company

Date Started: $\quad 06 / 19 / 78$

Date Completed: $\quad 06 / 26 / 78$

Diameter: $95 / 8$

Grade: J-55

Wt/Ft: 36

From: 0

To: 38

Cement: $72 \mathrm{Cu} . \mathrm{Ft}$.

Diameter: $51 / 2$

Grade: J-55

WtFt: 15.5

From: +1.29

To: 699

Cement: $335 \mathrm{Cu} . \mathrm{Ft}$.

Note: Bridge plug set at $641^{\prime}$. Perforated from $604^{\prime}$ to $631^{\prime}$ with 108 holes spaced at 4 holes per foot.

No plugging data.

STRATIGRAPHIC SUMMARY: Attached 
H-6c

Surficial Deposits

Gatuna Formation

Dockum Group

Dewey Lake Red Beds

Rustler Formation

Magenta Dolomite Member

Culebra Dolomite Member

Salado Formation

Upper Member

McNutt Member

Lower Member

Total Depth
$0-12$

12-38

NP

$38-427$

427-721

490-514

604-627

721-T.D.

741 
BOREHOLE:

OPERATOR:

PERMIT NO.:

LOCATION:

ELEVATION:

TOTAL DEPTH:

TYPE OF WELL:

DRILLER:

DRILLING RECORD:

CASING RECORD:
H-7a

Sandia National Labs

0.08-1271 (State Engineer's Office)

2495.04' FNL, 2492.35' FWL,

Sec. 14, T 23 S, R $30 \mathrm{E}$

$3164^{\prime}$

$154^{\prime}$

Hydrologic Test Hole

Pennsylvania Drilling Company

Date Started: $\quad 09 / 18 / 79$

Date Completed: $\quad 10 / 18 / 79$

Diameter: $133 / 8$

Grade:

Wt/Ft: 48

From:0

To: 38

Cement: $81 \mathrm{Cu} . \mathrm{Ft}$.

Diameter: 7

Grade:

WtFt: 23

From: 0

To: 109

Cement: $265 \mathrm{Cu} . \mathrm{Ft}$.

Note: Hole in standby condition for testing.

PLUGGING SCHEDULE:

No plugging data.

STRATIGRAPHIC SUMMARY: 
HYDROLOGIC TEST BOREHOLE DATA BASE

BOREHOLE:

OPERATOR:

PERMIT NO.:

LOCATION:

ELEVATION:

TOTAL DEPTH:

TYPE OF WELL:

DRILLER:

DRILLING RECORD:

CASING RECORD:

PLUGGING SCHEDULE:

STRATIGRAPHIC SUMMARY:

\section{H-7b1}

Sandia National Lab

0.08-1272 (State Engineer's Office)

2565.80' FNL, 2563.45' FWL,

Sec. 14, T 23 S, R 30 E

3164.17 (Top of Casing)

$286^{\prime}$

Hydrologic Test Hole

Pennsylvania Drilling Company

Date Started: $\quad 09 / 13 / 79$

Date Completed: $\quad 09 / 18 / 79$

Diameter: $133 / 8$

Grade:

Wt/Ft: 48

From: 0

To: 38

Cement: $54 \mathrm{Cu}$. Ft.

Diameter: 7

Grade:

Wt/Ft: 23

From: 0

To: 230

Cement: $270 \mathrm{Cu} . \mathrm{Ft}$.

Note: Hole in standby condition for testing.

No plugging data.

Attached 


\section{STRATIGRAPHIC SUMMARY}

H-7b1

Holocene

Surficial Deposits

$0-5$

Pleistocene

Gatuna Formation

12-38

Dockum Group

NP

Dewey Lake Red Beds

57-87

Rustler Formation

87-T.D.

Magenta Dolomite Member

$117-140$

Culebra Dolomite Member

$237-283$

Salado Formation

Upper Member

Lower Member

Castile Formation

Total Depth 


\section{HYDROLOGIC TEST BOREHOLE DATA BASE}

BOREHOLE:

OPERATOR:

PERMIT NO.:

LOCATION:

ELEVATION:

TOTAL DEPTH:

TYPE OF WELL:

DRILLER:

DRILLING RECORD:

CASING RECORD:

PLUGGING SCHEDULE:

STRATIGRAPHIC SUMMARY:
H-7b2

Sandia National Labs

---- (State Engineer's Office)

2662.16' FNL, 2537.98' FWL,

Sec. 14, T 23 S, R 30 E

$3164.40^{\prime}$ (Top of Casing)

295

Hydrologic Test Hole

Pennsylvania Drilling Company

Date Started: $\quad 08 / 27 / 83$

Date Completed: $\quad 09 / 02 / 83$

Diameter: $97 / 8$

Grade:

Wt/Ft:

From: 0

To: 20

Cement: Casing set in cement

Diameter: 7

Grade: J-55

Wt/Ft: 20

From: 0

To: 230.19

Cement: Cemented in place

Hole was back filled with peu gravel from $295^{\prime}$ to $268^{\prime}$ and left open for testing.

Attached 


\section{STRATIGRAPHIC SUMMARY}

Surficial Deposits

Gatuna Formation

Dockum Group

Dewey Lake Red Beds

Rustler Formation

Magenta Dolomite Member

Culebra Dolomite Member

Salado Formation

Upper Member

McNutt Member

Lower Member

Total Depth
57

NP

57-87

87-T.D.

117-140

232-280

295 


\section{HYDROLOGIC TEST BOREHOLE DATA BASE}

BOREHOLE:

OPERATOR:

PERMIT NO.:

LOCATION:

ELEVATION:

TOTAL DEPTH:

TYPE OF WELL:

DRILLER:

DRILLING RECORD:

CASING RECORD:
H-7c

Sandia National Labs

2591.93' FNL, 2467.51' FWL,

Sec. 14, T 23 S, R 30 E

3164.13 (Top of Casing)

$420^{\circ}$

Hydrologic Test Hole

Pennsylvania Drilling Company

Date Started: $\quad 09 / 06 / 79$

Date Completed: 11/02/79

Diameter: $133 / 8$

Grade:

Wt/Ft: 48

From: 0

To: 38

Cement: $68 \mathrm{Cu} . \mathrm{Ft}$.

Diameter: 7

Grade: J-55

Wt/Ft: 23

From: 0

To: 356

Cement: $706 \mathrm{Cu} . \mathrm{Ft}$.

Note: Hole in standby condition for testing. Slotted liner installed from $347^{\prime}$ to $420^{\prime}$.

PLUGGING SCHEDULE:

No plugging data.

STRATIGRAPHIC SUMMARY: Attached 


\section{STRATIGRAPHIC SUMMARY}

H-7c

Holocene

Unconsolidated Alluvium and Dune Sand

$0-5$

Pleistocene

Gatuna Formation

5-57

Permian

Dewey Lake Red Beds

$57-87$

Rustler Formation

$87-283$

Magenta Dolomite Member

$117-140$

Culebra Dolomite Member

237-273.5

Salado Formation

$283-420$

Dissolution Residue

Top of Salt Interval

283-405

405

Total Depth

420 


\section{HYDROLOGIC TEST BOREHOLE DATA BASE}

BOREHOLE:

OPERATOR:

PERMIT NO.:

LOCATION:

ELEVATION:

TOTAL DEPTH:

TYPE OF WELL:

DRILLER:

DRILLING RECORD:

CASING RECORD:

PLUGGING SCHEDULE:

STRATIGRAPHIC SUMMARY:
H-8a

Sandia National Labs

0.08.1274 (State Engineer's Office)

1962.61' FNL, 1486.59' FEL,

Sec. 23, T 24 S, R 30 E

3432.99' (Top of Casing)

$505^{\prime}$

Hydrologic Test Hole

Pennsylvania Drilling Company

Date Started: $\quad 09 / 07 / 79$

Date Completed: $\quad 09 / 18 / 79$

Diameter: $13 \cdot 3 / 8$

Grade:

Wt/Ft: 48

From: 0

To: 38

Cement: $108 \mathrm{Cu} . \mathrm{Ft}$.

Diameter: 7

Grade:

$\mathrm{Wt} / \mathrm{Ft}: 23$

From: 0

To: 452

Cement: 393

Note: Hole in standby condition for testing

No plugging data. 
BOREHOLE:

OPERATOR:

PERMIT NO.:

LOCATION:

ELEVATION:

TOTAL DEPTH:

TYPE OF WELL:

DRILLER:

DRILLING RECORD:

CASING RECORD:
H-8b

Sandia National Labs

0.08.1275 (State Engineer's Office)

1994.76' FNL, 1405.41' FEL,

Sec. 23, T 24 S, R 30 E

3433.64' (Top of Casing)

624

Hydrologic Test Hole

Pennsylvania Drilling Company

Date Started: $\quad 08 / 06 / 79$

Date Completed: $\quad 08 / 12 / 79$

Diameter: $133 / 8$

Grade:

$\mathrm{Wt} / \mathrm{Ft}: 48$

From: 0

To: 38

Cement: $108 \mathrm{Cu} . \mathrm{Ft}$.

$\therefore \quad$ Diameter: 7

Grade:

Wt/Ft: 20

From: 0

To: 574

Cement: $378 \mathrm{Cu} . \mathrm{Ft}$.

Note: Hole in standby condition for testing.

PLUGGING SCHEDULE: No plugging data.

STRATIGRAPHIC SUMMARY: Attached 


\section{STRATIGRAPHIC SUMMARY}

H-8b

Surficial Deposits

Gatuna Formation

Dockum Group

Dewey Lake Red Beds

Rustler Formation

Magenta Dolomite Member

Culebra Dolomite Member

Salado Formation

Upper Member

McNutt Member

Lower Member

Total Depth
$0-10$

10-153

NP

153-399

399-T.D.

466-490

586-613

624 
BOREHOLE:

OPERATOR:

PERMIT NO.:

LOCATION:

ELEVATION:

TOTAL DEPTH:

TYPE OF WELL:

DRILLER:

DRILLING RECORD:

CASING RECORD:

\section{H-8c}

Sandia National Labs

0.08.1276 (State Engineer's Office)

2059.36' FNL, 1470.14' FEL,

Sec. 23, T 24 S, R 30 E

$3432.90^{\prime}$ (Top of Casing)

$808^{\prime}$

Hydrologic Test Hole

Pennsylvania Drilling Company

Date Started: $\quad 07 / 27 / 79$

Date Completed: $\quad$ 08/06/79

Diameter: $133 / 8$

Grade:

$\mathrm{Wt} / \mathrm{Ft}: 48$

From: 0

To: 38

Cement: $108 \mathrm{Cu} . \mathrm{Ft}$.

Diameter: 7

Grade:

$\mathrm{Wt} / \mathrm{Ft}: 20$

From: 0

To: 734

Cement: $314 \mathrm{Cu}$. Ft.

Note: Hole is standby condition for testing.

PLUGGING SCHEDULE:

No plugging data.

STRATIGRAPHIC SUMMARY: Attached 


\section{STRATIGRAPHIC SUMMARY}

\section{H-8c}

\section{Holocene}

Unconsolidated Alluvium and Eolian Sand

$0-4$

\section{Pleistocene Rocks}

Mescalero Caliche

4-10

Gatuna Formation

$10-153$

Permian

Dewey Lake Red Beds

153-399

Rustler Formation

399-733

Magenta Dolomite Member

$466-488$

Culebra Dolomite Member

588-614

Salado Formation

$733-$

Dissolution Residue

733-774

MB 103

Top of Salt Interval

774-786

Total Depth 
BOREHOLE:

OPERATOR:

PERMIT NO.:

LOCATION:

ELEVATION:

TOTAL DEPTH:

TYPE OF WELL:

DRILLER:

DRILLING RECORD:

CASING RECORD:
H-9a

Sandia National Lab

0.08.1277 (State Engineer's Office)

2392.14' FNL, 138.92' FWL,

Sec. 4, T 24 S, R $31 \mathrm{E}$

3406.68' (Top of Casing)

$692^{\prime}$

Hydrologic Test Hole

Pennsylvania Drilling Company

Date Started: $\quad 07 / 09 / 79$

Date Completed: $\quad 08 / 23 / 83$

Diameter: $133 / 8$

Grade:

Wt/Ft: 48

From: 0

To: 38

Cement: $72 \mathrm{Cu} . \mathrm{Ft}$.

Diameter: 7

Grade: J-55

WtFt: 20

From: 0

To: 570

Cement: $266 \mathrm{Cu}$. Ft.

Diameter: 4.5" O.D.

Grade: J-55

Wt/Ft: 9.5

From: 0

To: 643

Cement:

Note: Hole in standby condition for testing.

PLUGGING SCHEDULE:

No plugging data.

STRATIGRAPHIC SUMMARY: Attached 


\section{H-9a}

\section{Holocene}

Surficial Deposits

Pleistocene

Gatuna Formation

Ochoan

Dewey Lake Red Beds

Rustler Formation

Forty-Niner Member

Magenta Dolomite Member

Tamarisk Member

Culebra Dolomite Member

Unnamed Part

Total Depth
$0-5$

$5-25$

25-455

455-T.D.

455-523

523-554

$554-647$

647-677

677-T.D.

692 


\section{HYDROLOGIC TEST BOREHOLE DATA BASE}

\section{BOREHOLE:}

OPERATOR:

PERMIT NO.:

LOCATION:

ELEVATION:

TOTAL DEPTH:

TYPE OF WELL:

DRILLER:

DRILLING RECORD:

CASING RECORD:

\section{H-9b}

Sandia National Labs

0.08 .1278 (State Engineer's Office)

2391.04' FNL, 238.63' FWL,

Sec. 4, T 24 S, R $31 \mathrm{E}$

3406.86' (Top of Casing)

$708^{\prime}$

Hydrologic Test Hole

Pennsylvania Drilling Company

Date Started: $08 / 14 / 79$

Date Completed: $\quad 08 / 28 / 79$

Diameter: $133 / 8$

Grade: K-55

Wt/Ft: 48

From: 0

To: 38

Cement: $72 \mathrm{Cu} \cdot \mathrm{Ft}$.

Diameter: 7

Grade: $]-55$

$\mathrm{Wt} / \mathrm{Ft}: 23$

From: 0

To: 638

Cement: $295 \mathrm{Cu}$. Ft.

Note: Hole in standby condition for testing.

PLUGGING SCHEDULE:

No plugging data.

STRATIGRAPHIC SUMMARY: Attached 


\section{STRATIGRAPHIC SUMMARY}

BOREHOLE

H-9b

\section{ROCK UNIT}

Surficial Deposits

Gatuna Formation

Dockum Group

Dewey Lake Red Beds

Rustler Formation

Magenta Dolomite Member

Culebra Dolomite Member

Salado Formation

Upper Member

McNutt Member

Lower Member

Total Depth
DEPTH INTERVAL IN FEET

$0-5$

5-25

NP

24-455

455-T.D.

523-554

647-677

708 
BOREHOLE:

OPERATOR:

PERMIT NO.:

LOCATION:

ELEVATION:

TOTAL DEPTH:

TYPE OF WELL:

DRILLER:

DRILLING RECORD:

CASING RECORD:

PLUGGING SCHEDULE:

STRATIGRAPHIC SUMMARY:
H-9c

Sandia National Labs

0.08.1279 (State Engineer's Office)

2479.06' FNL, 188.02' FWL,

Sec. 4, T 24 S, R 31 E

$3407.30^{\prime}$ (Top of Casing)

$816^{\circ}$

Hydrologic Test Hole

Pennsylvania Drilling Company

Date Started: $\quad 08 / 01 / 79$

Date Completed: $\quad 09 / 24 / 79$

Diameter: $133 / 8$

Grade: K-55

Wt/Ft: 48

From: 0

To: 38

Cement: $72 \mathrm{Cu} . \mathrm{Ft}$.

Diameter: 7

Grade: J-55

Wt/Ft: 20

From: 0

To: 783

Cement: $320 \mathrm{Cu}$. Ft.

Note: Hole in standby condition for testing.

No plugging data. 
HYDROLOGIC TEST BOREHOLE DATA BASE

BOREHOLE:

OPERATOR:

PERMIT NO.:

LOCATION:

ELEVATION:

TOTAL DEPTH:

TYPE OF WELL:

DRILLER:

DRILLING RECORD:

CASING RECORD:

PLUGGING SCHEDULE:

STRATIGRAPHIC SUMMARY:
H-10a

Sandia National Labs

0.08.1280 (State Engineer's Office)

433.0' FSL, 2068.9' FEL, Sec.

20, T $23 \mathrm{~S}, \mathrm{R} 32 \mathrm{E}$

3688.67' (Top of Casing)

$1318^{\prime}$

Hydrologic Test Hole

Pennsylvania Drilling Company

Date Started: $\quad 08 / 21 / 79$

Date Completed: $\quad 08 / 26 / 79$

Diameter: $133 / 8$

Grade: K-55

$\mathrm{Wt} / \mathrm{Ft}: 48$

From: 0

To: 38

Cement: $72 \mathrm{Cu}$. Ft.

Diameter: 7

Grade: J-55

Wt/Ft: 23

From: 0

To: 1243

Cement: $519 \mathrm{Cu} . \mathrm{Ft}$.

Note: Hole in standby condition for testing.

No plugging data. 
BOREHOLE:

OPERATOR:

PERMIT NO.:

LOCATION:

ELEVATION:

TOTAL DEPTH:

TYPE OF WELL:

DRILLER:

DRILLING RECORD:

CASING RECORD:
H-10b

Sandia National Labs

0.08.1281 (State Engineer's Office)

484.5' FSL, 1981.8' FEL, Sec.

20, T 23 S, R 32 E

$3689.47^{\prime}$ (Top of Casing)

$1398^{\prime}$

Hydrologic Test Hole

Pennsylvania Drilling Company

Date Started: $\quad 10 / 07 / 79$

Date Completed: $\quad 10 / 13 / 79$

Diameter: $133 / 8$

Grade:

Wt/Ft: 48

From: 0

To: 38

Cement: $72 \mathrm{Cu} . \mathrm{Ft}$.

Diameter: 7

Grade: J-55

Wt/Ft: 23

From: 0

To: 1346

Cement: $480 \mathrm{Cu} . \mathrm{Ft}$.

Note: Hole in standby condition for possible future tests.

PLUGGING SCHEDULE:

No plugging data.

STRATIGRAPHIC SUMMARY: 


\section{HYDROLOGIC TEST BOREHOLE DATA BASE}

BOREHOLE:

OPERATOR:

PERMIT NO.:

LOCATION:

ELEVATION:

TOTAL DEPTH:

TYPE OF WELL:

DRILLER:

DRILLING RECORD:

CASING RECORD:
H-10c

Sandia National Labs

0.08.1282 (State Engineer's Office)

384.5' FSL, 1981.8 FEL, Sec.

20, T 23 S, R 32 E

$3687^{\prime}$

$1550^{\circ}$

Hydrologic Test Hole

Pennsylvania Drilling Company

Date Started: $\quad 08 / 11 / 79$

Date Completed: $\quad 08 / 20 / 79$

Diameter: $133 / 8$

Grade: K-55

WtFt: 48

From: 0

To: 38

Cement: $72 \mathrm{Cu} . \mathrm{Ft}$.

Diameter: 7

Grade:

$\mathrm{Wt} / \mathrm{Ft}: 20$

From: 0

To: 1483

Cement: $627 \mathrm{Cu}$. Ft.

Note: Hole in standby condition for testing.

PLUGGING SCHEDULE: No plugging data.

STRATIGRAPHIC SUMMARY: Attached 


\section{STRATIGRAPHIC SUMMARY}

H-10c

Holocene

Unconsolidated Alluvium and Eolian Sand

$0-5$

Pleistocene

Mescalero Caliche

$5-9$

Gatuna Formation

9-90

Triassic

Dockum Group

Chinle Formation

$90-482$

Santa Rosa Sandstone

$482-658$

Permian

Dewey Lake Red Beds

658-1204

Rustler Formation

1204-1501

Magenta Dolomite Member

1256-1280

Culebra Dolomite Member

1360-1387

Salado Formation

1501

Top of Salt Interval

1501

Total Depth

1538 
HYDROLOGIC TEST BOREHOLE DATA BASE

BOREHOLE:

OPERATOR:

PERMIT NO.:

LOCATION:

ELEVATION:

TOTAL DEPTH:

TYPE OF WELL:

DRILLER:

DRILLING RECORD:

CASING RECORD:

PLUGGING SCHEDULE:

STRATIGRAPHIC SUMMARY:

\section{H-11b2}

Sandia National Labs

0.08 .1462 (State Engineer's Office)

1436.3' FSL, 168.7' FEL, Sec.

33, T 22 S, R $31 \mathrm{E}$

3411.64' (Top of Casing)

$776^{\prime}$

Hydrologic Test Hole

Pennsylvania Drilling Company

Date Started: $\quad 10 / 83$

Date Completed: $11 / 28 / 83$

Diameter: $95 / 8$

Grade: $\mathrm{H}-40$

Wt/Ft: 40

From: 0

To: 37

Cement:

Diameter: $51 / 2$

Grade: J-55

Wt/Ft: 15.5

From: 0

To: 733.39 .

Cement: Cemented

Note: $43 / 4$ " open hole from $733.39^{\prime}$ to the total depth of 776 .

No plugging data.

Attached 
STRATIGRAPHIC SUMMARY

DEPTH INTERVAL IN FEET

H-11b2

Magenta Dolomite Member

618-644

Culebra Dolomite Member

733-757

Total Depth

776 
BOREHOLE:

OPERATOR:

PERMIT NO.:

LOCATION:

ELEVATION:

TOTAL DEPTH:

TYPE OF WELL:

DRILLER:

DRILLING RECORD:

CASING RECORD:
H-11b3

Sandia National Labs

0.08.1462 (State Engineer's Office)

1501.7' FSL, 105.2' FEL, Sec.

$33, \mathrm{~T} 22 \mathrm{~S}, \mathrm{R} 31 \mathrm{E}$

3412.42' (Top of Casing)

788.7' (Below Kelly Bushing)

Hydrologic Test Hole

Pennsylvania Drilling Company

Date Started: $\quad 12 / 01 / 83$

Date Completed: $01 / 84$

Diameter: $95 / 8$

Grade: H-30

Wt/Ft: 40

From: 0

To: 34

Cement:

Diameter: $51 / 2$

Grade: J-55

Wt/Ft: 55

From: 0

To: 733

Cement:

Note: $43 / 4^{\prime \prime}$ open hole from $733^{\prime}$ to the total depth of $788.7^{\prime}$.

No plugging data.

STRATIGRAPHIC SUMMARY: Attached 


\section{STRATIGRAPHIC SUMMARY}

H-11b3

Surficial Deposits

Gatuna Formation

Dockum Group

Dewey Lake Red Beds

Rustler Formation

Magenta Dolomite Member

616-644

Culebra Dolomite Member

734-759

Salado Formation

Upper Member

McNutt Member

Lower Member

Total Depth

788.7 


\section{HYDROLOGIC TEST BOREHOLE DATA BASE}

BOREHOLE:

OPERATOR:

PERMIT NO.:

LOCATION:

ELEVATION:

TOTAL DEPTH:

TYPE OF WELL:

DRILLER:

DRILLING RECORD:

CASING RECORD:

PLUGGING SCHEDULE:

STRATIGRAPHIC SUMMARY:
H-11b4

Sandia National Labs

0.08.1474 (State Engineer's Office)

1514.7' FSL, 320.2' FEL, Sec.

33, T 22 S, R $31 \mathrm{E}$

$3410.89^{\circ}$ (Top of Casing)

$765^{\circ}$

Hydrologic Test Hole

Pennsylvania Drilling Company

Date Started: $\quad 02 / 23 / 88$

Date Completed: $\quad 03 / 17 / 88$

Diameter: $85 / 8$

Grade: $\mathrm{H}-40$

Wt/Ft: 28

From: 0

To: 27

Cement:

Diameter: $51 / 2$

Grade: J-55

Wt/Ft: 15.5

From: 0

To: 714

Cement:

Note: $43 / 4$ " open hole from $715^{\prime}$ to total depth of $765^{\prime}$.

No plugging data. 
BOREHOLE:

OPERATOR:

PERMIT NO.:

LOCATION:

ELEVATION:

TOTAL DEPTH:

TYPE OF WELL:

DRILLER:

DRILLING RECORD:

CASING RECORD:

PLUGGING SCHEDULE:

STRATIGRAPHIC SUMMARY: 'Attached

H-12

$1001^{\prime}$

From: 0

To: 37

To: 820

Cement:
Sandia National Labs

0.08.1463 (State Engineer's Office)

23.1' FNL, 91.9' FEL,

Sec. 15, T 23 S, R $31 \mathrm{E}$

$3427.19^{\prime}$ (Top of Casing)

Hydrologic Test Hole

Pennsylvania Drilling Company

Date Started: $\quad 10 / 03 / 83$

Date Completed: $\quad 10 / 18 / 83$

Diameter: $95 / 8$

Grade: $\mathrm{H}-40$

Wt/Ft: 36

Cement: $63 \mathrm{Cu} . \mathrm{Ft}$.

Diameter: $51 / 2$

Grade: J-55

Wt/Ft: 15.5

From: +1.45

Note: $43 / 4$ " open hole from $820^{\prime}$ to plugged back depth of $890^{\prime}$.

No plugging data. 


\title{
STRATIGRAPHIC SUMMARY
}

\author{
Quaternary Deposits \\ Dockum Group \\ Dewey Lake Red Beds \\ Rustler Formation \\ Forty-Niner Member \\ Magenta Dolomite Member \\ Tamarisk Member \\ Culebra Dolomite Member \\ Unnamed Lower Member \\ Salado Formation \\ Unnamed Upper Member \\ Total Depth
}

$0-10+$

$10+-70$

$70-622$

$622-976$

$622-678$

678-703

703-823

823-850

850-976

976-T.D.

976-T.D.

1001 


\section{HYDROLOGIC TEST BOREHOLE DATA BASE}

BOREHOLE:

OPERATOR:

PERMIT NO:

LOCATION:

ELEVATION:

TOTAL DEPTH:

TYPE OF WELL:

DRILLER:

DRILLING RECORD:

CASING RECORD:

\section{H-14}

Sandia National Labs

0.08.1469 (State Engineer's Office)

372.2' FSL, 562.4' FWL, Sec.

29, T 22 S, R 31 E

3347.11' (Top of Casing)

$589^{\prime}$

Hydrologic Test Hole

Pennsylvania Drilling Company

Date Started: $\quad 10 / 86$

Date Completed: $\quad 10 / 86$

Diameter: 8.625

Grade:

WtFt: 28

From: +1.6

To: 39

Cement:

Diameter: 5.5

Grade:

Wt/Ft: 15.5

From: 0

To: 532 .

Cement:

PLUGGING SCHEDULE:

No plugging data.

STRATIGRAPHIC SUMMARY:

Attached 


\section{STRATIGRAPHIC SUMMARY}

Surficial Deposits

Gatuna Formation

Dockum Group

Dewey Lake Red Beds

Rustler Formation

Magenta Dolomite Member

Culebra Dolomite Member

Salado Formation

Upper Member

McNutt Member

Lower Member

Total Depth
$0-13$

13-40

NP

40-360

360-T.D.

424-448

$545-572$

589 
BOREHOLE:

OPERATOR:

PERMIT NO.:

LOCATION:

ELEVATION:

TOTAL DEPTH:

TYPE OF WELL:

DRILLER:

DRILLING RECORD:

CASING RECORD:

\section{H-15}

Sandia National Labs

0.08.1470 (State Engineer's Office)

88.7' FNL, 174.3' FEL, Sec.

$28, \mathrm{~T} 22 \mathrm{~S}, \mathrm{R} 31 \mathrm{E}$

3481.63' (Top of Casing)

$900^{\prime}$

Hydrologic Test Hole

Pennsylvania Drilling Company

Date Started: $11 / 86$

Date Completed: $11 / 86$

Diameter: 8.625

Grade:

Wt/Ft: 28

From: 0

To: 39

Diameter: 5.5

Grade:

WUFt: 15.5

From: +1.4

To: 853

PLUGGING SCHEDULE: No plugging data.

STRATIGRAPHIC SUMMARY: Attached 
STRATIGRAPHIC SUMMARY

BOREHOLE

H-15
ROCK UNIT

Surficial Deposits

Gatuna Formation

Dockum Group

Dewey Lake Red Beds

Rustler Formation

Magenta Dolomite Member

Culebra Dolomite Member

Salado Formation

Upper Member

McNutt Member

Lower Member

Total Depth
DEPTH INTERVAL IN FEET

0-8

4-42

$42-168$

168-692

692-T.D.

748-773

861-883

900 
BOREHOLE:

OPERATOR:

PERMIT NO.:

LOCATION:

ELEVATION:

TOTAL DEPTH:

TYPE OF WELL:

DRILLER:

DRILLING RECORD:

CASING RECORD:
H-16

Sandia National Labs

0.08.1471 (State Engineer's Office)

1112.6 FSL, 1241.3 FEL, Sec.

20, T 22 S, R $31 \mathrm{E}$

$3406.77^{\prime}$ (Top of Casing)

$850.9^{\prime}$

Hydrologic Test Hole

Pennsylvania Drilling Company

Date Started: $\quad 07 / 13 / 87$

Date Completed: $\quad 08 / 18 / 87$

Diameter: $103 / 4$

Grade:

Wt/Ft: 40.5

From: 0

To: 36.5

Diameter: 7

Grade:

Wt/Ft: 23

From: 0

To: 469

Note: $61 / 8^{\prime \prime}$ inch open hole from $469^{\prime}$ to total depth of $850.9^{\prime}$.

PLUGGING SCHEDULE:

No plugging data.

STRATIGRAPHIC SUMMARY: 


\section{HYDROLOGIC TEST BOREHOLE DATA BASE}

BOREHOLE:

OPERATOR:

PERMIT NO.:

LOCATION:

ELEVATION:

TOTAL DEPTH:

TYPE OF WELL:

DRILLER:

DRILLING RECORD:

CASING RECORD:

PLUGGING SCHEDULE:

STRATIGRAPHIC SUMMARY:
H-17

Sandia National Labs

0.08.1477 (State Engineer's Office)

1465.5' FSL, 994.1' FWL, Sec.

3, T 23 S, R $31 \mathrm{E}$

3385.31' (Top of Casing)

$880^{\prime}$

Hydrologic Test Hole

Pennsylvania Drilling Company

Date Started: $\quad 09 / 21 / 87$

Date Completed: $\quad 11 / 04 / 87$

Diameter: $103 / 4$

Grade: $\mathrm{H}-40$

$\mathrm{Wt} / \mathrm{Ft}: 40.5$

From: 0

To: 38

Diameter: 7

Grade: J-55

Wt/Ft: 23

From: 0

To: 692

Note: $61 / 8^{\prime \prime}$ inch open hole from $693^{\prime}$ to the plugged back depth of $773^{\prime}$.

The borehole was plugged back with cement grout to a total depth of $773^{\prime}$ on 11/06/87.

Attached 


\section{STRATIGRAPHIC SUMMARY}

H-17

Quaternary Deposits

Holocene

$0-15$

Pleistocene

Mescalero Caliche

$15-21.5$

Upper Triassic

Dockum Group

21.5-55

Upper Permian

Dewey Lake Red Beds

Rustler Formation

55-509

Forty-Niner Member

$509-855.7$

Magenta Dolomite Member

509:564

Tamarisk Member

564-590.8

Culebra Dolomite Member

$590.8-705.8$

Unnamed Lower Member

705.8-731.4

$731.4-855.7$

Salado Formation

855.7-870.3+ 
HYDROLOGIC TEST BOREHOLE DATA BASE

\section{BOREHOLE:}

OPERATOR:

PERMIT NO.:

LOCATION:

ELEVATION:

TOTAL DEPTH:

TYPE OF WELL:

DRILLER:

DRILLING RECORD:

CASING RECORD:

\section{H-18}

Sandia National Labs

0.08.1473 (State Engineer's Office)

964.8' FNL, 445.6' FWL, Sec.

20, T 22 S, R $31 \mathrm{E}$

3414.21' (Top of Casing)

$840^{\circ}$

Hydrologic Test Hole

Pennsylvania Drilling Company

Date Started: $\quad 09 / 29 / 87$

Date Completed: $\quad 11 / 16 / 87$

Diameter: $103 / 4$

Grade: $\mathrm{H}-40$

Wt/Ft: 40.5

From: 0

To: 39

Diameter: 7

Grade: J-55

Wt/Ft: 23

From: 0

To: 673

Note: $61 / 8^{\prime \prime}$ inch open hole from $673^{\prime}$ to the plugged back depth of $766^{\prime}$.

PLUGGING SCHEDULE:

The borehole was plugged back with cement grout to a total depth of $766^{\prime}$ on $11 / 19 / 87$.

STRATIGRAPHIC SUMMARY: Attached 
STRATIGRAPHIC SUMMARY

H-18

Quaternary Deposits

Holocene

Drill Pad Material \& Unconsolidated Sand

$0-5$

Pleistocene

Mescalero Caliche

$5-8$

Upper Triassic

Dockum Group

8-20

Upper Permian

Dewey Lake Red Beds

20-506.1

Rustler Formation

$506.1-820.9$

Forty-Niner Member

$506.1-571.2$

Magenta Dolomite

$571.2-594.2$

Tamarisk Member

594.2-688.6

Culebra Dolomite Member

688.6-712.8

Unnamed Lower Member

$712.8-820.9$

Salado Formation 820.9-830.5+ 


\section{POTASH BOREHOLE DATA BASE}

BOREHOLE:

OPERATOR:

PERMIT NO.:

LOCATION:

ELEVATION:

TOTAL DEPTH:

TYPE OF WELL:

DRILLER:

DRILLING RECORD:

CASING RECORD:

WATER HORIZONS:

RUSTLER FORMATION:

TOP OF SALADO:

MARKER BED 124:

PLUGGING SCHEDULE:
P-1

U.S. Department of Energy

Unknown

$327^{\prime}$ FSL, 551' FWL of Sec. 29, SW 1/4

Sec. 29, T 22 S, R 31 E

$3345^{\prime}$

$1591^{\prime}$

Potash Core Test

Pennsylvania Drilling Company (for Sandia Laboratories)

Date Started: $\quad 08 / 23 / 76$

Date Completed: $\quad 09 / 02 / 76$

Conventional Rotary Drilling procedures were used to bore to the top of the potash-bearing section, and consecutive cores, $2-1 / 4$ " in diameter, were taken through the full thickness of the potash-bearing section.

4-1/2" Casing: Unknown

Recovered: 203' of casing left

in hole between 591-794' below

land surface.

Unknown

358-677

677

1477-1486

From: $1591^{\circ}$

To: $0^{\prime}$

Int: $1591^{\prime}$

Material: Cement

STRATIGRAPHIC SUMMARY: Attached 


\section{STRATIGRAPHIC SUMMARY}

Pleistocene Rocks

Gatuna Formation

$10-40$

Permian Rocks

Dewey Lake Red Beds

40-358

Rustler Formation

358-677

Magenta Dolomite Member

$423-448$

Culebra Dolomite Member

538-565

Salado Formation

$677-1591$

Upper Member

677-1191

McNutt Potash Zone

1191-1583

Vaca Triste Sandstone Member

1191-1201

11 th Ore Zone

1246-1250

MB 117

1259-1262

MB 118

1282-1285

MB 119

1307-1309

10th Ore Zone

1319-1324

MB 120

1334-1335

9th Ore Zone

1338-1343

MB 121

1347-1349

MB 122

1356-1357

8th Ore Zone

1361-1370

Union Anhydrite

1381-1393

7th Ore Zone

1400-1404

6th Ore Zone

1414-1416

5th Ore Zone

1419-1432

MB 123

1462-1469

MB 124

1477-1486

4th Ore Zone

1490-1503

3d Ore Zone

1511-1526

2d Ore Zone

1533-1538

1st Ore Zone

1554-1562

MB 126

1582-1583

Lower Member

1583-1587 


\section{POTASH BOREHOLE DATA BASE}

BOREHOLE:

OPERATOR:

PERMIT NO.:

LOCATION:

ELEVATION:

TOTAL DEPTH:

TYPE OF WELL:

DRILLER:

DRILLING RECORD:

CASING RECORD:

WATER HORIZONS:

RUSTLER FORMATION:

TOP OF SALADO:

MARKER BED 124:

PLUGGiNG SCHEDULE:
P-2

U.S. Department of Energy

Unknown

$125^{\prime}$ FNL, 172' FEL of Sec. 28, NE1/4

Sec. 28, T 22 S, R $31 \mathrm{E}$

$3478^{\prime}$

$1895^{\prime}$

Potash Core Test

Boyles Bros. Drilling Company (for Sandia Laboratories)

Date Started: $\quad 08 / 25 / 76$

Date Completed: $\quad 09 / 02 / 76$

Conventional Rotary Drilling procedures were used to bore to the top of the potash-bearing section, and consecutive cores, $2-1 / 4^{\prime \prime}$ in diameter, were taken through the full thickness of the potash-bearing section.

4-1/2" Casing: Unknown

Recovered: All

Unknown

690-1008'

$1008^{\prime}$

$1787-1795^{\circ}$

From: $1895^{\circ}$

To: $0^{\prime}$

Int: $1895^{\prime}$

Material: Cement

STRATIGRAPHIC SUMMARY: Attached 


\section{STRATIGRAPHIC SUMMARY}

Pleistocene Rocks

Gatuna Formation

18-38

Triassic Rocks

Santa Rosa Sandstone

$38-164$

Permian Rocks

Dewey Lake Red Beds

$164-690$

Rustler Formation

690-1008

Magenta Dolomite Member

$748-773$

Culebra Dolomite Member

$857-883$

Salado Formation

1008-1895

Upper Member

McNutt Potash Zone

1008-1506

Vaca Triste Sandstone Member

1506-1883

11th Ore Zone

1506-1512

1562-1565

MB 117

1574- 1576

MB 118

1599-1601

MB 119

1622-1626

10th Ore Zone

1632-1639

MB 120

1646-1647

9th Ore Zone

1652-1656

MB 121

MB 122

1662-1663

1670-1671

8th Ore Zone

1678-1687

Union Anhydrite

1695-1705

7 th Ore Zone

1712-1719

6th Ore Zone

1731-1733

5th Ore Zone

1738-1745

MB 123

1774-1781

MB 124

1787-1795

4th Ore Zone

1799-1809

3d Ore Zone

1818-1832

2d Ore Zone

1836-1840

1st Ore Zone

1859-1870

MB 126

1882-1883

Lower Member

1883-1894 


\section{POTASH BOREHOLE DATA BASE}

BOREHOLE:

OPERATOR:

PERMIT NO.:

LOCATION:

ELEVATION:

TOTAL DEPTH:

TYPE OF WELL:

DRILLER:

DRILLING RECORD:

CASING RECORD:

WATER HORIZONS:

RUSTLER FORMATION:

TOP OF SALADO:

MARKER BED 124:

PLUGGING SCHEDULE:
P-3

U.S. Department of Energy

Unknown

103' FSL, 3122' FEL of Sec. 20, SW1/4

Sec. 20, T 22 S, R 31 E

$3382^{\prime}$

1676

Potash Core Test

Pennsylvania Drilling Company (for Sandia Laboratories)

Date Started: $\quad 08 / 26 / 76$

Date Completed: $\quad 09 / 07 / 76$

Conventional Rotary Drilling procedures were used to bore to the top of the potash-bearing section, and consecutive cores, $2-1 / 4^{\text {" }}$ in diameter, were taken through the full thickness of the potash-bearing section.

4-1/2" Casing: Unknown

Recovered: 336 of casing left

in hole between $490-826^{\prime}$ below

land surface.

Unknown

468-786

$786^{\circ}$

1571-1579

From: $1676^{\circ}$

To: $0^{\prime}$

Int: $1676^{\circ}$

Material: Cement

Attached 


\section{STRATIGRAPHIC SUMMARY}

BOREHOLE

P-3
ROCK UNIT

Holocene Deposits

Pleistocene rocks

Gatuna Formation

Permian Rocks

Dewey Lake Red Beds

Rustler Formation

Magenta Dolomite Member

Culebra Dolomite Member

Salado Formation

Upper Member

McNutt Potash Zone

Vaca Triste Sandstone Member

11 th Ore Zone

MB 117

MB 118

MB 119

10th Ore Zone

MB 120

9th Ore Zone

MB 121

MB 122

8th Ore Zone

Union Anhydrite

7th Ore Zone

6th Ore Zone

5 th Ore Zone

MB 123

MB 124

4th Ore Zone

3d Ore Zone

2d Ore Zone

Ist Ore Zone

MB 126
DEPTH INTERVAL IN FEET

$0-10$

$10-41$

41-468

468-786

529-553

$642-665$

786-1668

$786-1287$

1287-1668

1287-1295

1346-1349

1357-1358

1375-1378

1405-1407

$1415-1420$

1428-1429

1434-1438

1443-1445

1452-1453

1458-1467

1473-1481

1494-1499

1509-1511

1515-1525

1555-1563

1571-1579

1585-1595

1599-1617

1623-1627

1645-1656

1667-1668 


\section{POTASH BOREHOLE DATA BASE}

BOREHOLE:

OPERATOR:

PERMIT NO.:

LOCATION:

ELEVATION:

TOTAL DEPTH:

TYPE OF WELL:

DRILLER:

DRILLING RECORD:

CASING RECORD:

WATER HORIZONS:

RUSTLER FORMATION:

TOP OF SALADO:

MARKER BED 124:

PLUGGING SCHEDULE:
P.4

U.S. Department of Energy

Unknown

146' FSL, 1487' FEL of Sec. 28, SE1/4

Sec. 28, T 22 S, R 31 E

$3441^{\prime}$

$1857^{\prime}$

Potash Core Test

Boyles Bros. Drilling Company (for Sandia Laboratories)

Date Started: $\quad 08 / 28 / 76$

Date Completed: $\quad 09 / 04 / 76$

Conventional Rotary Drilling procedures were used to bore to the top of the potash-bearing section, and consecutive cores, $2-1 / 4$ " in diameter, were taken through the full thickness of the potash-bearing section.

4-1/2" Casing: Unknown

Recovered: All

Driller reported water at $850^{\prime}$.

$609-930^{\circ}$

930'

1742-1752'

From: 1857

To: $0^{\prime}$

Int: $1857^{\circ}$

Material: Cement

STRATIGRAPHIC SUMMARY: Attached 


\section{STRATIGRAPHIC SUMMARY}

\section{BOREHOLE}

P-4
ROCK UNIT

Holocene Deposits

Triassic Rocks

Santa Rosa Sandstone

Permian Rocks

Dewey Lake Red Beds

Rustler Formation

Magenta Dolomite Member

Culebra Dolomite Member

Salado Formation

Upper Member

McNutt Potash Zone

Vaca Triste Sandstone Member

11 th Ore Zone

MB 117

MB 118

MB 119

10th Ore Zone

MB 120

9th Ore Zone

MB 121

MB 122

8th Ore Zone

Union Anhydrite

7th Ore Zone

6th Ore Zone

5th Ore Zone

MB 123

MB 124

4th Ore Zone

3d Ore Zone

2d Ore Zone

1st Ore Zone

MB 126
DEPTH INTERVAL IN FEET

0-8

8-99

99-609

$609-930$

662-686

775-802

930-1857

930-1446

1446-1853

1446-1452

1506-1509

1519-1521

1544-1547

1570-1572

1581-1589

1596-1597

1603-1607

1610-1612

1620-1621

1628-1637

1646-1659

1667-1671

1683-1686

1690-1700

1728-1735

1742-1752

1756-1768

1777-1792

1798-1803

1824-1835

1852-1853 


\section{POTASH BOREHOLE DATA BASE}

BOREHOLE:

OPERATOR:

PERMIT NO.:

LOCATION:

ELEVATION:

TOTAL DEPTH:

TYPE OF WELL:

DRILLER:

DRILLING RECORD:

CASING RECORD:

WATER HORIZONS:

RUSTLER FORMATION:

TOP OF SALADO:

MARKER BED 124:

PLUGGING SCHEDULE:
P-5

U.S. Department of Energy

Unknown

202' FSL, 165' FEL of Sec. 17, SE1/4

Sec. 17, T 22 S, R $31 \mathrm{E}$

$3472^{\prime}$

$1830^{\circ}$

Potash Core Test

Pennsylvania Drilling Company (for Sandia Laboratories)

Date Started: $09 / 10 / 76 \quad$ Date Completed: $09 / 21 / 76$

Conventional Rotary Drilling procedures were used to bore to the top of the potash-bearing section, and consecutive cores, $2-1 / 4$ " in diameter, were taken through the full thickness of the potash-bearing section.

4-1/2" Casing: Unknown

Recovered: 568 ' of casing left

in hole between 435-1003'

below land surface.

Unknown

623-947'

$947^{\prime}$

1695-1705

From: $1830^{\prime}$

To: $0^{\circ}$

Int: $1830^{\prime}$

Material: Cement

STRATIGRAPHIC SUMMARY: Attached 


\section{STRATIGRAPHIC SUMMARY}

Triassic Rocks

Santa Rosa Sandstone

Permian Rocks

Dewey Lake Red Beds $\quad$ 146-623

Rustler Formation

Magenta Dolomite Member

686-711

Culebra Dolomite Member

804-827

Salado Formation

947-1830

Upper Member

947-1429

McNutt Potash Zone

1429-1785

Vaca Triste Sandstone Member

1429-1436

11 th Ore Zone

1482-1486

MB 117

1492-1494

MB 118

1514-1517

MB 119

1541-1543

10th Ore Zone

1550-1556

MB 120

1560-1561

9th Ore Zone

1567-1571

MB 121

1573-1575

MB 122

1580-1582

8th Ore Zone

1589-1595

Union Anhydrite

1604-1611

7th Ore Zone

1623-1628

6th Ore Zone

1635-1638

5th Ore Zone

1643-1659

MB 123

1680-1687

MB 124

1695-1705

4th Ore Zone

1709-1717

3d Ore Zone

1725-1737

1 st Ore Zone

1742-1746

MB 126

1784-1785

Lower Member

MB 127

1785-1830

MB 128

1810-1811

1821-1822 


\section{POTASH BOREHOLE DATA BASE}

BOREHOLE:

OPERATOR:

PERMIT NO.:

LOCATION:

ELEVATION:

TOTAL DEPTH:

TYPE OF WELL:

DRILLER:

DRILLING RECORD:

CASING RECORD:

WATER HORIZONS:

RUSTLER FORMATION:

TOP OF SALADO:

MARKER BED 124:

PLUGGING SCHEDULE:
P-6

U.S. Department of Energy

Unknown

2767' FSL, 199' FWL of Sec. 30, NW1/4

Sec. $30, T 22$ S, R $31 \mathrm{E}$

$3354^{\circ}$

1573

Potash Core Test

Boyles Bros. Drilling Company (for Sandia Laboratories)

Date Started: $\quad 09 / 03 / 76$

Date Completed: $\quad 09 / 16 / 76$

Conventional Rotary Drilling procedures were used to bore to the top of the potash-bearing section, and consecutive cores, $2-1 / 4^{\prime \prime}$ in diameter, were taken through the full thickness of the potash-bearing section.

4-1/2" Casing: Unknown

Recovered: All

Unknown

$357-659^{\prime}$

$659^{\prime}$

$1453-1462^{\prime}$

From: $1573^{\prime}$

To: 0 '

Int: $1573^{\prime}$

Material: Cement

STRATIGRAPHIC SUMMARY: Attached 


\section{STRATIGRAPHIC SUMMARY}

BOREHOLE

P-6

\section{ROCK UNIT}

Holocene Deposits

Pleistocene Rocks

Gatuna Formation

Permian Rocks

Dewey Lake Red Beds

Rustler Formation

Magenta Dolomite Member

Culebra Dolomite Member

Salado Formation

Upper Member

McNutt Potash Zone

Vaca Triste Sandstone Member

11 th Ore Zone

MB 117

MB 118

MB 119

10th Ore Zone

MB 120

9th Ore Zone

MB 121

MB 122

8th Ore Zone

Union Anhydrite

7th Ore Zone

6th Ore Zone

5th Ore Zone

MB 123

MB 124

4th Ore Zone

3d Ore Zone

2d Ore Zone

1st Ore Zone

MB 126
DEPTH INTERVAL IN FEET

0-8

$8-18$

18-357

$357-659$

$417-443$

$537-560$

$659-1573$

$659-1162$

$1162-1560$

$1162-1170$

1218-1221

1231-1233

1252-1256

1279-1281

1291-1296

1307-1308

1313-1317

1324-1326

1332-1333

1338-1348

1357-1365

1378-1382

1394-1396

1400-1410

1436-1443

1453-1462

1468-1481

1489-1502

1509-1513

1533-1543

1559-1560 


\section{POTASH BOREHOLE DATA BASE}

BOREHOLE:

OPERATOR:

PERMIT NO.:

LOCATION:

ELEVATION:

TOTAL DEPTH:

TYPE OF WELL:

DRILLER:

DRILLING RECORD:

CASING RECORD:

WATER HORIZONS:

RUSTLER FORMATION:

TOP OF SALADO:

MARKER BED 124:

PLUGGING SCHEDULE:
P-7

U.S. Department of Energy

Unknown

513' FNL, 396' FWL of Sec. 5, NW1/4

Sec. 5, T 23 S, R $31 \mathrm{E}$

$3332^{\prime}$

$1574^{\prime}$

Potash Core Test

Pennsylvania Drilling Company (for Sandia Laboratories)

Date Started: 09/04/76 Date Completed: $09 / 21 / 76$

Remarks: Encountered air pocket at $980^{\circ}$ below land surface, and lost casing seat.

Encountered several air pockets between 980-1264'. Lost mud at 1234'.

Conventional Rotary Drilling procedures were used to bore to the top of the potash-bearing section, and consecutive cores, 2-1/4" in diameter, were taken through the full thickness of the potash-bearing section.

4-1/2" Casing: Unknown

Recovered: $210^{\prime}$ of casing left

in hole between $530-740^{\circ}$ below

land surface.

Unknown

$312-630^{\prime}$

$630^{\prime}$

$1448-1459^{\prime}$

From: $1830^{\prime}$

To: $0^{\prime}$

Int: $1830^{\prime}$

Material: Cement

STRATIGRAPHIC SUMMARY: Attached 


\section{STRATIGRAPHIC SUMMARY}

DEPTH INTERVAL IN FEET

\section{P-7}

Holocene Deposits

Pleistocene Rocks

Gatuna Formation

Permian Rocks

Dewey Lake Red Beds

Rustler Formation

Magenta Dolomite Member

Culebra Dolomite Member

Salado Formation

Upper Member

McNutt Potash Zone

Vaca Triste Sandstone Member

11 th Ore Zone

MB 117

MB 118

MB 119

10th Ore Zone

MB 120

9th Ore Zone

MB 121

MB 122

8th Ore Zone

Union Anhydrite

7th Ore Zone

6th Ore Zone

5th Ore Zone

MB 123

MB 124

4th Ore Zone

3d Ore Zone

2d Ore Zone

1st Ore Zone

MB 126

\section{$0-11$}

$11-45$

45-312

312-630

373-398

496-522

$630-1574$

630-1155

$1155-1566$

$1155-1162$

$1215-1220$

1228-1230

1252-1256

$1277-1279$

1291-1296

1307-1308

$1312-1316$

1326-1328

1330-1331

1335-1346

$1358-1372$

1377-1382

1391-1393

1398-1407

1433-1441

1448-1459

1467-1484

1492-1507

1513-1518

1537-1547

1565-1566 


\section{POTASH BOREHOLE DATA BASE}

BOREHOLE:

OPERATOR:

PERMIT NO.:

LOCATION:

ELEVATION:

TOTAL DEPTH:

TYPE OF WELL:

DRILLER:

DRILLING RECORD:

CASING RECORD:

WATER HORIZONS:

RUSTLER FORMATION:

TOP OF SALADO:

MARKER BED 124:

PLUGGING SCHEDULE:
P-8

U.S. Department of Energy

Unknown

642' FNL, 96' FWL of Sec. 4, NW1/4

Sec. 4, T 23 S, R 31 E

3336

$1660^{\prime}$

Potash Core Test

Boyles Bros. Drilling Company (for Sandia Laboratories)

Date Started: $\quad 09 / 08 / 76$

Date Completed: 09/15/76

Conventional Rotary Drilling procedures were used to bore to the top of the potash-bearing section, and consecutive cores, $2-1 / 4$ " in diameter, were taken through the full thickness of the potash-bearing section.

4-1/2" Casing: Unknown

Recovered: All

No water reported by driller.

391-715'

715

$1537-1545^{\prime}$

From: $1660^{\prime}$

To: $0^{\prime}$

Int: $1660^{\prime}$

Material: Cement

STRATIGRAPHIC SUMMARY: Attached 


\section{STRATIGRAPHIC SUMMARY}

BOREHOLE

P-8
ROCK UNIT

Holocene Deposits

Pleistocene Rocks

Gatuna Formation

Permian Rocks

Dewey Lake Red Beds

Rustler Formation

Magenta Dolomite Member

Culebra Dolomite Member

Salado Formation

Upper Member

McNutt Potash Zone

Vaca Triste Sandstone Member 11 th Ore Zone

MB 117

MB 118

MB 119

10th Ore Zone

MB 120

9th Ore Zone

MB 121

MB 122

8th Ore Zone

Union Anhydrite

7th Ore Zone

6th Ore Zone

5th Ore Zone

MB 123

MB 124

4th Ore Zone

3d Ore Zone

2d Ore Zone

1st Ore Zone

MB 126
DEPTH INTERVAL IN FEET

$0-9$

9-39

39-391

391-715

450-474

563-585

715-1660

715-1237

1237-1652

1237-1245

1297-1301

1308-1310

1333-1337

1360-1362

1373-1380

1389-1390

1395-1399

1405-1407

1413-1414

1421-1430

1440-1455

1461-1465

1472-1475

1481-1492

1517-1524

1537-1545

1554-1567

1577-1596

1601-1604

1624-1633

1661-1662 
BOREHOLE:

OPERATOR:

PERMIT NO.:

LOCATION:

ELEVATION:

TOTAL DEPTH:

TYPE OF WELL:

DRILLER:

DRILLING RECORD:

CASING RECORD:

WATER HORIZONS: RUSTLER FORMATION: TOP OF SALADO: MARKER BED 124:

PLUGGING SCHEDULE:
P.9

U.S. Department of Energy

Unknown

1493' FSL, 143' FEL of Sec. 33, SE1/4

Sec. 33, T 22 S, R 31 E

$3409^{\prime}$

$1796^{\prime}$

Potash Core Test

Boyles Bros. Drilling Company (for Sandia Laboratories)

Date Started: $\quad 09 / 16 / 76$

Date Completed: $\quad 09 / 25 / 76$

Conventional Rotary Drilling procedures were used to bore to the top of the potash-bearing section, and consecutive cores, $2-1 / 4$ " in diameter, were taken through the full thickness of the potash-bearing section.

4-1/2" Casing: Unknown

Recovered: All

Driller reported water at $220^{\prime}$ below land surface, making about $25 \mathrm{gpm}$. $562-881^{\prime}$

$881^{\prime}$

$1686-1695$

From: $1796^{\circ}$

To: $0^{\prime}$

Int: $1796^{\circ}$

Material: Cement

STRATIGRAPHIC SUMMARY: Attached 


\section{STRATIGRAPHIC SUMMARY}

\section{BOREHOLE} P-9
ROCK UNIT

Holocene Deposit

Triassic Rocks

Santa Rosa Sandstone

Permian Rocks

Dewey Lake Red Beds

Rustler Formation

Magenta Dolomite Member

Culebra Dolomite Member

Salado Formation

Upper Member

McNutt Potash Zone

Vaca Triste Sandstone Member

11 th Ore Zone

MB 117

MB 118

MB 119

10th Ore Zone

MB 120

9th Ore Zone

MB 121

MB 122

8th Ore Zone

Union Anhydrite

7 th Ore Zone

6th Ore Zone

5 th Ore Zone

MB 123

MB 124

4th Ore Zone

3d Ore Zone

2d Ore Zone

1st Ore Zone

MB 126
DEPTH INTERVAL IN FEET

\section{$0-11$}

$11-66$

66-562

$562-881$

$617-644$

734-757

881-1796

881-1401

1401-1796

$1401-1410$

1458-1462

1471-1473

1496-1499

$1519-1521$

1530-1538

1546-1547

1552-1555

1561-1563

1569-1570

$1577-1585$

1597-1608

1613-1618

1626-1629

1634-1643

1668-1676

1686-1695

1699-1714

1723-1738

1744-1748

1769-1778

1795-1796 


\section{POTASH BOREHOLE DATA BASE}

BOREHOLE:

OPERATOR:

PERMIT NO.:

LOCATION:

ELEVATION:

TOTAL DEPTH:

TYPE OF WELL:

DRILLER:

DRILLING RECORD:

CASING RECORD:

WATER HORIZONS:

RUSTLER FORMATION:

TOP OF SALADO:

MARKER BED 124:

PLUGGING SCHEDULE:
P-10

U.S. Department of Energy

Unknown

$2315^{\prime}$ FNL, 339' FWL of Sec. 26, NW1/4

Sec. 26, T 22 S, R 31 E

$3508^{\prime}$

2009'

Potash Core Test

Pennsylvania Drilling Company (for Sandia Laboratories)

Date Started: $\quad 09 / 24 / 76$

Date Completed: $\quad 10 / 15 / 76$

Conventional Rotary Drilling procedures were used to bore to the top of the potash-bearing section, and consecutive cores, $2-1 / 4$ " in diameter, were taken through the full thickness of the potash-bearing section.

4-1/2" Casing: Unknown

Recovered: All

Unknown

686-1086

$1086^{\prime}$

$1880-1888^{\prime}$

From: 2009'

To: $0^{\prime}$

Int: 2009'

Material: Cement

STRATIGRAPHIC SUMMARY: Attached 
STRATIGRAPHIC SUMMARY

BOREHOLE

P-10
ROCK UNIT

Holocene Deposits

Triassic Rocks

Santa Rosa Sandstone

Permian Rocks

Dewey Lake Red Beds

Rustler Formation

Magenta Dolomite Member

Culebra Dolomite Member

Salado Formation

Upper Member

McNutt Potash Zone

Vaca Triste Sandstone Member

11 th Ore Zone

MB 117

MB 118

MB 119

10th Ore Zone

MB 120

9th Ore Zone

MB 121

MB 122

8th Ore Zone

Union Anhydrite

7th Ore Zone

6th Ore Zone

5th Ore Zone

MB 123

MB 124

4th Ore Zone

3d Ore Zone

2d Ore Zone

1st Ore Zone

MB 126

Lower Member

MB 127
DEPTH INTERVAL IN FEET

$0-8$

8-151

151-686

686-1086

$757-781$

931-957

1086-2009

1086-1594

1594-1983

1594-1603

1652-1655

1662-1664

1686-1688

1710-1712

1717-1725

1733-1734

1740-1744

1751-1753

1759-1760

1765-1775

1784-1798

1807-1811

1822-1825

1831-1841

1868-1875

1880-1888

1892-1905

1913-1929

1934-1938

1961-1969

1982-1983

1983-2009

2005-2008 
BOREHOLE:

OPERATOR:

PERMIT NO.:

LOCATION:

ELEVATION:

TOTAL DEPTH:

TYPE OF WELL:

DRILLER:

DRILLING RECORD:

CASING RECORD:

WATER HORIZONS:

RUSTLER FORMATION:

TOP OF SALADO:

MARKER BED 124:

PLUGGING SCHEDULE:

\section{P-11}

U.S. Department of Energy

Unknown

$175^{\prime} \mathrm{FNL}$ and 177' FWL of Sec. 23, NW1/4

Sec. 23, T 22 S, R 31 E

$3506^{\prime}$

$1940^{\circ}$

Potash Core Test

Pennsylvania Drilling Company (for Sandia Laboratories)

Date Started: $\quad 09 / 24 / 76$

Date Completed: $\quad 10 / 16 / 76$

Conventional Rotary Drilling procedures were used to bore to the top of the potash-bearing section, and consecutive cores, $2-1 / 4$ " in diameter, were taken through the full thickness of the potash-bearing section.

4-1/2" Casing: Unknown

Recovered: All

Unknown

745-1058'

$1058^{\prime}$

$1824-1833^{\prime}$

From: $1940^{\prime}$

To: $0^{\prime}$

Int: $1940^{\prime}$

Material: Cement

STRATIGRAPHIC SUMMARY: Attached 


\section{STRATIGRAPHIC SUMMARY}

Triassic Rocks

Santa Rosa Sandstone

$9-224$

Permian Rocks

Dewey Lake Red Beds

224-745

Rustler Formation

745-1058

Magenta Dolomite Member

798-823

Culebra Dolomite Member

912-938

Salado Formation

Upper Member

McNutt Potash Zone

1058-1942

1058-1550

Vaca Triste Sandstone Member

1550-1917

11th Ore Zone

1550-1557

MB 117

MB 118

1604-1608

1616-1618

MB 119

1640-1642

10th Ore Zone

1664-1666

1674-1682

MB 120

1687-1688

9th Ore Zone

1693-1698

MB 121

MB 122

8th Ore Zone

1702-1704

1711-1712

Union Anhydrite

1717-1724

1735-1740

7th Ore Zone

6th Ore Zone

1754-1759

1767-1770

5th Ore Zone

1775-1785

MB 123

1811-1818

MB 124

1824-1833

4th Ore Zone

1837-1846

3d Ore Zone

1853-1866

2d Ore Zone

1871-1874

1st Ore Zone

1890-1901

MB 126

1916-1917

Lower Member

1917-1942

MB 127

1940-1941 
BOREHOLE:

OPERATOR:

PERMIT NO.:

LOCATION:

ELEVATION:

TOTAL DEPTH:

TYPE OF WELL:

DRILLER:

DRILLING RECORD:

CASING RECORD:

WATER HORIZONS:

RUSTLER FORMATION:

TOP OF SALADO:

MARKER BED 124:

PLUGGING SCHEDULE:
P-12

U.S. Department of Energy

Unknown

167' FNL, 195' FEL of Sec. 24, NE1/4

Sec. $24, \mathrm{~T} 22 \mathrm{~S}, \mathrm{R} 30 \mathrm{E}$

3376

$1598^{\prime}$

Potash Core Test

Pennsylvania Drilling Company (for Sandia Laboratories)

Date Started: $\quad 09 / 17 / 76$

Date Completed: $10 / 20 / 76$

Remarks: Lost circulation at $742^{\prime}$ and $813^{\prime}$ below land.

Conventional Rotary Drilling procedures were used to bore to the top of the

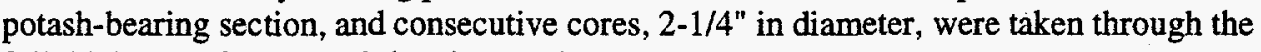
full thickness of the potash-bearing section.

4-1/2" Casing: Unknown

Recovered: All

Unknown

461-749:

$749^{\prime}$

$1494-1509^{\prime}$

From: $1598^{\prime}$

To: $0^{\prime}$

Int: 1598

Material: Cement

STRATIGRAPHIC SUMMARY: Attached 


\section{STRATIGRAPHIC SUMMARY}

BOREHOLE

P-12
ROCK UNIT

Holocene Deposits

Permian Rocks

Dewey Lake Red Beds

Rustler Formation

Magenta Dolomite Member

Culebra Dolomite Member

Salado Formation

Upper Member

McNutt Potash Zone

Vaca Triste Sandstone Member

11th Ore Zone

MB 117

MB 118

MB 119

10th Ore Zone

MB 120

9th Ore Zone

MB 121

MB 122

8th Ore Zone

Union Anhydrite

7th Ore Zone

6th Ore Zone

5th Ore Zone

MB 123

MB 124

4th Ore Zone

3d Ore Zone

2d Ore Zone

1st Ore Zone

MB 126
DEPTH INTERVAL IN FEET

0-8

8-461

461-749

$519-543$

633-656

749-1598

749-1226

1226-1597

1226-1233

1280-1284

1290-1292

1314-1317

1338-1340

1346-1353

1361-1362

$1367-1370$

1376-1378

1384-1385

1390-1398

1407-1417

1432-1436

1442-1445

1450-1459

1486-1492

1494-1509

1514-1523

1533-1546

1550-1555

1572-1582

1596-1597 


\section{POTASH BOREHOLE DATA BASE}

BOREHOLE:

OPERATOR:

PERMIT NO.:

LOCATION:

ELEVATION:

TOTAL DEPTH:

TYPE OF WELL:

DRILLER:

DRILLING RECORD:

CASING RECORD:

WATER HORIZONS:

RUSTLER FORMATION:

TOP OF SALADO:

MARKER BED 124:

PLUGGING SCHEDULE:
P-13

U.S. Department of Energy

Unknown

125' FNL, 116' FWL of Sec. 18, NW1/4

Sec. 18, T 22 S, R $31 \mathrm{E}$

$3345^{\prime}$

$1576^{\prime}$

Potash Core Test

Boyles Bros. Drilling Company (for Sandia Laboratories)

Date Started: $\quad 09 / 17 / 76$

Date Completed: $\quad 09 / 23 / 76$

Conventional Rotary Drilling procedures were used to bore to the top of the potash-bearing section, and consecutive cores, $2-1 / 4$ " in diameter, were taken through the full thickness of the potash-bearing section.

\section{4-1/2" Casing: Unknown}

Recovered: All

Hit water at $630^{\prime}$.

427-721'

$721^{\prime}$

$1462-1471^{\prime}$

From: 1576

To: $0^{\prime}$

Int: 1576

Material: Cement

STRATIGRAPHIC SUMMARY:

Attached 


\section{STRATIGRAPHIC SUMMARY}

Pleistocene Rocks

Gatuna Formation

$12-38$

\section{Permian Rocks}

Dewey Lake Red Beds

$38-427$

Rustler Formation

$427-721$

Magenta Dolomite Member

490-514

Culebra Dolomite Member

604-627

Salado Formation

721-1573

Upper Member

721-1201

McNutt Potash Zone

1201-1547

Vaca Triste Sandstone Member

1201-1208

11th Ore Zone

1252-1255

MB 117

1264-1265

MB 118

1287-1289

MB 119

1309-1311

10th Ore Zone

MB 120

9th Ore Zone

MB 121

MB 122

8th Ore Zone

Union Anhydrite

7th Ore Zone

6th Ore Zone

5th Ore Zone

MB 123

MB 124

4th Ore Zone

3d Ore Zone

2d Ore Zone

lst Ore Zone

MB 126

Lower Member

MB 127
1317-1323

1330-1331

1336-1340

1344-1346

1355-1356

1359-1368

1377-1382

1395-1400

1407-1410

1413-1423

1447-1453

1462-1471

1474-1483

1491-1501

1506-1510

1525-1533

1547-1548

1548-1573

1572-1573 


\section{POTASH BOREHOLE DATA BASE}

BOREHOLE:

OPERATOR:

PERMIT NO.:

LOCATION:

ELEVATION:

TOTAL DEPTH:

TYPE OF WELL:

DRILLER:

DRILLING RECORD:

CASING RECORD:

WATER HORIZONS:

RUSTLER FORMATION:

TOP OF SALADO:

MARKER BED 124:

PLUGGING SCHEDULE:
P-14

U.S. Department of Energy

Unknown

307' FSL, 615.8' FWL of Sec. 24, SW1/4

Sec. 24, T 22 S, R 30 E

$3358^{\prime}$

$1545^{\prime}$

Potash Core Test

Boyles Bros. Drilling Company (for Sandia Laboratories)

Date Started: $\quad 09 / 24 / 76$

Date Completed: $\quad 10 / 03 / 76$

Conventional Rotary Drilling procedures were used to bore to the top of the potash-bearing section, and consecutive cores, 2-1/4" in diameter, were taken through the full thickness of the potash-bearing section.

4-1/2" Casing: Unknown

Recovered: All

Hit water at 589".

$387-687^{\prime}$

$687^{\prime}$

$1410-1419^{\prime}$

From: $1545^{\prime}$

To: $775^{\prime}$

Int: $770^{\prime}$

Material: Cement

From: $775^{\circ}$

To: $0^{\prime}$

Int: $775^{\prime}$

Material: Well

Note: Hole plugged from $1545^{\prime}-775^{\prime}$ with cement and converted to hydrologic observation well in Rustler Formation.

STRATIGRAPHC SUMMARY: Attached 


\section{STRATIGRAPHIC SUMMARY}

BOREHOLE

P-14
ROCK UNIT

Holocene Deposits

Pleistocene Rocks

Gatuna Formation

Permian Rocks

Dewey Lake Red Beds

Rustler Formation

Magenta Dolomite Member

Culebra Dolomite Member

Salado Formation

Upper Member

McNutt Potash Zone

Vaca Triste Sandstone Member

11 th Ore Zone

MB 117

MB 118

MB 119

10th Ore Zone

MB 120

9th Ore Zone

MB 121

MB 122

8th Ore Zone

Union Anhydrite

7th Ore Zone

6th Ore Zone

5th Ore Zone

MB 123

MB 124

4th Ore Zone

3d Ore Zone

2d Ore Zone

1st Ore Zone

MB 126

Lower Member

MB 127
DEPTH INTERVAL IN FEET

$0-10$

$10-42$

42-387

$387-687$

453-475

573-595

$687-1540$

687-1133

1133-1510

1133-1141

1186-1190

1199-1200

1225-1228

1251-1253

1257-1263

1273-1274

1277-1282

1287-1289

1297-1298

1302-1310

1319-1328

1342-1345

1352-1355

1361-1370

1394-1400

1410-1419

1423-1435

1443-1455

1461-1465

1482-1492

1509-1510

1510-1540

1534-1536 


\section{POTASH BOREHOLE DATA BASE}

\section{BOREHOLE:}

OPERATOR:

PERMIT NO.:

LOCATION:

ELEVATION:

TOTAL DEPTH:

TYPE OF WELL:

DRILLER:

DRILLING RECORD:

CASING RECORD:

WATER HORIZONS:

RUSTLER FORMATION:

TOP OF SALADO:

MARKER BED 124:

PLUGGING SCHEDULE:
P-15

U.S. Department of Energy

Unknown

410.8' FSL, 1.92.32' FWL of Sec. 21, SW1/4

Sec. 31, T 22 S, R 31 E

$3309.7^{\prime}$

$1465^{\circ}$

Potash Core Test

Boyles Bros. Drilling Company (for Sandia Laboratories)

Date Started: 10/04/76 Date Completed: $10 / 14 / 76$

Conventional Rotary Drilling procedures were used to bore to the top of the potash-bearing section, and consecutive cores, $2-1 / 4$ " in diameter, were taken through the full thickness of the potash-bearing section.

4-1/2": Casing Unknown

Recovered: $210^{\prime}$ of casing left

in hole between $530-740^{\circ}$. below

land surface.

From: $1465^{\prime}$

To: $620^{\circ}$

Int: $845^{\prime}$

Material: Cement

From: $620^{\circ}$

To: $0^{\prime}$

Int: $620^{\circ}$

Material: Well

Note: Hole plugged from $1465^{\prime}-620^{\prime}$ with cement, and converted to hydrologic observation well in Rustler Formation.

STRATIGRAPHIC SUMMARY: Attached 


\section{STRATIGRAPHIC SUMMARY}

Holocene Deposits

$0-11$

Pleistocene Rocks

Gatuna Formation

$11-32$

Permian Rocks

Dewey Lake Red Beds

$32-231$

Rustler Formation

231-542

Magenta Dolomite Member

294-321

Culebra Dolomite Member

413-435

Salado Formation

$542-1465$

Upper Member

$542-1057$

McNutt Potash Zone

1057-1453

Vaca Triste Sandstone Member

1057-1065

11th Ore Zone

1116-1119

MB 117

MB 118

1128-1130

MB 119

1149-1152

1176-1178

10th Ore Zone

1187-1195

MB 120

1203-1204

9th Ore Zone

MB 121

1208-1212

MB 122

1216-1218

8th Ore Zone

1225-1226

Union Anhydrite

1234-1244

7 th Ore Zone

1251-1263

6th Ore Zone

1271-1275

5th Ore Zone

1284-1288

1292-1301

MB 123

1326-1333

MB 124

1343-1353

4th Ore Zone

1361-1376

3d Ore Zone

1384-1397

2d Ore Zone

1404-1408

1st Ore Zone

1426-1436

MB 126

1452-1453 


\section{POTASH BOREHOLE DATA BASE}

BOREHOLE:

OPERATOR:

PERMIT NO.:

LOCATION:

ELEVATION:

TOTAL DEPTH:

TYPE OF WELL:

DRILLER:

DRILLING RECORD:

CASING RECORD:

WATER HORIZONS:

RUSTLER FORMATION:

TOP OF SALADO:

MARKER BED 124:

PLUGGING SCHEDULE:
P.16

U.S. Department of Energy

Unknown

951' FSL, 1629' FEL of Sec. 5, SW1/4

Sec. 5, T 23S, R 31E

$3323^{\prime}$

$1585^{\prime}$

Potash Core Test

Boyles Bros. Drilling Company (for Sandia Laboratories)

Date Started: $\quad 09 / 27 / 76$

Date Completed: $10 / 05 / 76$

Conventional Rotary Drilling procedures were used to bore to the top of the potash bearing section, and consecutive cores, $2-1 / 4$ " in diameter, were taken through the full thickness of the potash-bearing section.

4-1/2" Casing: Unknown

Recovered: All

Unknown

$316-646^{\prime}$

$646^{\prime}$

$1460-1470^{\prime \prime}$

From: $1585^{\circ}$

To: 0 '

Int: $1585^{\prime}$

Material: Cement

STRATIGRAPHIC SUMMARY: Attached 


\section{STRATIGRAPHIC SUMMARY}

Pleistocene Rocks

Gatuna Formation

14-32

Permian Rocks

Dewey Lake Red Beds

32-316

Rustler Formation

316-646

Magenta Dolomite Member

$376-401$

Culebra Dolomite Member

500-523

Salado Formation

646-1587

Upper Member

646-1174

McNutt Potash Zone

1174-1585

Vaca Triste Sandstone Member

1174-1182

11th Ore Zone

1235-1237

MB 117

1245-1247

MB 118

1269-1273

MB 119

1293-1295

10th Ore Zone

1307-1313

MB 120

1323-1324

9th Ore Zone

MB 121

1328-1331

MB 122

1336-1338

8th Ore Zone

$1345-1346$

Union Anhydrite

1352-1365

7 th Ore Zone

1373-1390

6th Ore Zone

1396-1400

5th Ore Zone

1411-1412

1417-1425

MB 123

1450-1456

MB 124

1460-1470

4th Ore Zone

1480-1501

3d Ore Zone

1510-1526

2d Ore Zone

1533-1536

1st Ore Zone

1556-1568

MB 126

1583-1585 


\section{POTASH BOREHOLE DATA BASE}

BOREHOLE:

OPERATOR:

PERMIT NO.:

LOCATION:

ELEVATION:

TOTAL DEPTH:

TYPE OF WELL:

DRILLER:

DRILLING RECORD:

CASING RECORD:

WATER HORIZONS: RUSTLER FORMATION:

TOP OF SALADO:

MARKER BED 124:

PLUGGING SCHEDULE:
P-17

U.S. Department of Energy

Unknown

1372.2' FSL, 401.9' FWL of Sec. 4, SW1/4

Sec. 4, T 23 S, R 31 E

$3340^{\circ}$

$1660^{\prime}$

Potash Core Test

Boyles Bros. Drilling Company (for Sandia Laboratories)

Date Started: $\quad 10 / 18 / 76$

Date Completed: $10 / 26 / 76$

Conventional Rotary Drilling procedures were used to bore to the top of the potash-bearing section, and consecutive cores, $2-1 / 4^{\prime \prime}$ in diameter, were taken through the full thickness of the potash-bearing section.

4-1/2" Casing: Unknown

Recovered: Unknown

Hit water at $265^{\prime}$ and at $600^{\circ}$.

$382-715^{\prime}$

$715^{\prime}$

$1527-1535^{\prime}$

From: $1660^{\prime}$

To: $731^{\prime}$

Int: 929'

Material: Cement

From: $731^{\prime}$

To: $0^{\prime}$

Int: $731^{\prime}$

Material: Well

Note: Hole plugged from $1660^{\prime}-731$ with cement and converted to hydrologic observation well in Rustler Formation.

STRATIGRAPHIC SUMMARY: Attached 


\section{STRATIGRAPHIC SUMMARY}

Pleistocene Rocks

Gatuna Formation

$14-46$

Permian Rocks

Dewey Lake Red Beds

46-382

Rustler Formation

$382-715$

Magenta Dolomite Member

$438-463$

Culebra Dolomite Member

558-583

Salado Formation

715-1662

Upper Member

$715-1234$

McNutt Potash Zone

1234-1648

Vaca Triste Sandstone Member

1234-1242

11th Ore Zone

1294-1299

MB 117

1306-1308

MB 118

1330-1334

MB 119

1358-1359

10th Ore Zone

1368-1376

MB 120

1387-1388.

9th Ore Zone

MB 121

MB 122

1391-1396

1402-1404

8th Ore Zone

Union Anhydrite

1410-1411

1418-1428

7 th Ore Zone

1438-1453

6th Ore Zone

1456-1461

5th Ore Zone

1471-1474

1478-1487

MB 123

1513-1521

MB 124

1527-1535

4th Ore Zone

1544-1563

3d Ore Zone

1573-1587

2d Ore Zone

1594-1599

1st Ore Zone

1619-1630

MB 126

1647-1648 


\section{POTASH BOREHOLE DATA BASE}

BOREHOLE:

OPERATOR:

PERMIT NO.:

LOCATION:

ELEVATION:

TOTAL DEPTH:

TYPE OF WELL:

DRILLER:

DRILLING RECORD:

CASING RECORD:

WATER HORIZONS: RUSTLER FORMATION: TOP OF SALADO:

MARKER BED 124:

PLUGGING SCHEDULE:
P-18

U.S. Department of Energy

Unknown

138.5' FSL, 732.7' FEL of Sec. 26, SE1/4

Sec. 26, T 22 S, R 31 E

$3479^{\prime}$

$1998^{\prime}$

Potash Core Test

Pennsylvania Drilling Company (for Sandia Laboratories)

Date Started: $\quad 10 / 19 / 76$

Date Completed: 11/05/76

Conventional Rotary Drilling procedures were used to bore to the top of the potash-bearing section, and consecutive cores, $2-1 / 4$ " in diameter, were taken through the full thickness of the potash-bearing section.

4-1/2" Casing: Unknown

Recovered: Unknown

Unknown

626-1088

$1088^{\prime}$

$1880-1889^{\prime}$

From: $1998^{\circ}$

To: $1125^{\prime}$

Int: $873^{\prime}$

Material: Cement

From: $1125^{\prime}$

To: $0^{\prime}$

Int: 1125

Material: Well

Note: Hole plugged from $1998-1125^{\prime}$ with cement, and converted to hydrologic observation well in Rustler Formation.

STRATIGRAPHIC SUMMARY: Attached 


\section{STRATIGRAPHIC SUMMARY}

BOREHOLE P-18
ROCK UNIT

Holocene Deposits

Triassic Rocks

Santa Rosa Sandstone

Permian Rocks

Dewey Lake Red Beds

Rustler Formation

Magenta Dolomite Member

Culebra Dolomite Member

Salado Formation

Upper Member

McNutt Potash Zone

Vaca Triste Sandstone Member

11th Ore Zone

MB 117

MB 118

MB 119

10th Ore Zone

MB 120

9th Ore Zone

MB 121

MB 122

8th Ore Zone

Union Anhydrite

7 th Ore Zone

6th Ore Zone

5th Ore Zone

MB 123

MB 124

4th Ore Zone

3d Ore Zone

2d Ore Zone

1st Ore Zone

MB 126
DEPTH INTERVAL IN FEET

0-9

9-87

$87-626$

626-1088

704-730

909-938

1088-2000

1088-1604

1604-1987

1604-1614

1658-1662

$1670-1672$

1692-1694

$1716-1718$

1726-1734

1741-1742

1749-1752

1756-1758

1765-1766

1772-1783

1793-1808

1812-1817

1828-1830

1835-1844

1868-1875

1880-1889

1897-1910

1919-1933

1938-1942

1961-1972

1986-1987 


\section{POTASH BOREHOLE DATA BASE}

BOREHOLE:

OPERATOR:

PERMIT NO.:

LOCATION:

ELEVATION:

TOTAL DEPTH:

TYPE OF WELL:

DRILLER:

DRILLING RECORD:

CASING RECORD:

WATER HORIZONS;

RUSTLER FORMATION:

TOP OF SALADO:

MARKER BED 124:

PLUGGING SCHEDULE:
P-19

U.S. Department of Energy

Unknown

1652' FSL, 2330' FWL of Sec. 23, SW1/4

Sec. 23, T 22 S, R 31 E

$3546.3^{\prime}$

$2000^{\prime}$

Potash Core Test

Pennsylvania Drilling Company (for Sandia Laboratories)

Date Started: $\quad 10 / 19 / 76$

Date Completed: $11 / 04 / 76$

Conventional Rotary Drilling procedures were used to bore to the top of the potash-bearing section, and consecutive cores, 2-1/4" in diameter, were taken through the full thickness of the potash-bearing section.

4-1/2" Casing: Unknown

Recovered: All

From: $2000^{\prime}$

To: $0^{\prime}$

Int: $2000^{\prime}$

Material: Cement

STRATIGRAPHIC SUMMARY: Attached 


\section{STRATIGRAPHIC SUMMARY}

BOREHOLE

P-19
ROCK UNIT

Holocene Deposits

Triassic Rocks

Santa Rosa Sandstone

Permian Rocks

Dewey Lake Red Beds

Rustler Formation

Magenta Dolomite Member

Culebra Dolomite Member

Salado Formation

Upper Member

McNutt Potash Zone

Vaca Triste Sandstone Member

11 th Ore Zone

MB 117

MB 118

MB 119

10th Ore Zone

MB 120

9th Ore Zone

MB 121

MB 122

8th Ore Zone

Union Anhydrite

7th Ore Zone

6th Ore Zone

5th Ore Zone

MB 123

MB 124

4th Ore Zone

3d Ore Zone

2d Ore Zone

1st Ore Zone
DEPTH INTERVAL IN FEET

0-8

8-232

232-758

$758-1117$

814-839

967-997

1117-2002

$1117-1621$

1621-2011

1621-1628

1677-1681

1688-1690

1711-1713

1735-1737

1745-1753

1760-1761

1767-1771

1776-1778

1785-1786

1792-1801

1812-1822

1835-1840

1850-1854

1858-1872

1892-1901

1909-1917

1923-1933

1944-1955

1962-1967

1983-1994 


\section{POTASH BOREHOLE DATA BASE}

BOREHOLE:

OPERATOR:

PERMIT NO.:

LOCATION:

ELEVATION:

TOTAL DEPTH:

TYPE OF WELL:

DRILLER:

DRILLING RECORD:

CASING RECORD:

WATER HORIZONS:

RUSTLER FORMATION:

TOP OF SALADO:

MARKER BED 124:

PLUGGING SCHEDULE:
P-20

U.S. Department of Energy

Unknown

794' FSL, 103' FEL of Sec. 14, SE1/4

Sec. 14, T 22 S, R $31 \mathrm{E}$

$3552.9^{\prime}$

$1995^{\prime}$

Potash Core Test

Boyles Bros. Drilling Company (for Sandia Laboratories)

Date Started: $\quad 10 / 06 / 76$

Date Completed: $\quad 10 / 14 / 76$

Remarks: Very weak air flow noted during logging operation on October 14,1976 . Air pocket escaped detection throughout coring operation.

Conventional Rotary Drilling procedures were used to bore to the top of the potash-bearing section, and consecutive cores, $2-1 / 4^{\prime \prime}$ in diameter, were taken through the full thickness of the potash-bearing section.

4-1/2" Casing: Unknown

Recovered: All

From: $1995^{\prime}$

To: $0^{\prime}$

Int: $1995^{\circ}$

Material: Cement

STRATIGRAPHIC SUMMARY: Attached 


\section{STRATIGRAPHIC SUMMARY}

Triassic Rocks

Santa Rosa Sandstone

$6-261$

\section{Permian Rocks}

Dewey Lake Red Beds

261-780

Rustler Formation

780-1103

Magenta Dolomite Member

$839-866$

Culebra Dolomite Member

953-979

Salado Formation

1103-1996

Upper Member

McNutt Potash Zone

1103-1604

Vaca Triste Sandstone Member

1604-1977

11th Ore Zone

1604-1612

MB 117

1658-1662

MB 118

1671-1673

MB 119

1696-1697

10th Ore Zone

1720-1721

MB 120

1728-1735

9th Ore Zone

1742-1743

MB 121

1747-1750

MB 122

1756-1758

8th Ore Zone

1764-1765

Union Anhydrite

1771-1779

7th Ore Zone

1789-1795

6th Ore Zone

1807-1811

5th Ore Zone

1821-1823

MB 123

1828-1839

MB 124

1866-1873

4th Ore Zone

1880-1891

3d Ore Zone

1893-1904

2d Ore Zone

1911-1924

1st Ore Zone

1928-1932

1947-1961

MB 126

1975-1977

Lower Member

1977-1996 


\section{POTASH BOREHOLE DATA BASE}

BOREHOLE:

OPERATOR:

PERMIT NO.:

LOCATION:

ELEVATION:

TOTAL DEPTH:

TYPE OF WELL:

DRILLER:

DRILLING RECORD:

CASING RECORD:

WATER HORIZONS:

RUSTLER FORMATION:

TOP OF SALADO:

MARKER BED 124:

PLUGGING SCHEDULE:
P-21

U.S. Department of Energy

Unknown

$852^{\prime}$ FNL, 150' FEL of Sec. 15, NE1/4

Sec. 15, T 22 S, R $31 \mathrm{E}$

$3510^{\prime}$

$1915^{\prime}$

Potash Core Test

Boyles Bros. Drilling Company (for Sandia Laboratories)

Date Started: $\quad 10 / 15 / 76$

Date Completed: $\quad 10 / 26 / 76$

Conventional Rotary Drilling procedures were used to bore to the top of the potash-bearing section, and consecutive cores, $2-1 / 4$ " in diameter, were taken through the full thickness of the potash-bearing section.

4-1/2" Casing: Unknown

Recovered: All

Driller reported water between 525-595'.

734-1043'

$1043^{\prime}$

$1796-1805^{\prime}$

From: $1915^{\prime}$

To: $0^{\prime}$

Int: 1915

Material: Cement

STRATIGRAPHIC SUMMARY: Attached 


\section{STRATIGRAPHIC SUMMARY}

\section{BOREHOLE}

P-21
ROCK UNIT

Holocene Deposits

Triassic Rocks

Santa Rosa Sandstone

Permian Rocks

Dewey Lake Red Beds

Rustler Formation

Magenta Dolomite Member

Culebra Dolomite Member

Salado Formation

Upper Member

McNutt Potash Zone

Vaca Triste Sandstone Member

11 th Ore Zone

MB 117

MB 118

MB 119

10th Ore Zone

MB 120

9th Ore Zone

MB 121

MB 122

8th Ore Zone

Union Anhydrite

7th Ore Zone

6th Ore Zone

5th Ore Zone

MB 123

MB 124

4th Ore Zone

3d Ore Zone

2d Ore Zone

1st Ore Zone

MB 126

Lower Member

MB 127
DEPTH INTERVAL IN FEET

$0-8$

8-225

225-734

734-1043

788-812

899-924

1043-1918

1043-1526

1526-1887

1526-1533

1579-1583

1591-1593

1615-1616

1637-1638

1647-1653

1659-1660

1665-1669

1676-1678

1685-1686

1690-1699

1707-1714

1727-1732

1740-1744

1748-1757

1781-1789

1796-1805

1811-1817

1824-1837

1841-1845

1859-1873

1886-1887

1887-1918

1912-1913 
BOREHOLE:

OPERATOR:

PERMIT NO.:

LOCATION:

ELEVATION:

TOTAL DEPTH:

TYPE OF WELL:

DRILLER:

DRILLING RECORD:

CASING RECORD:

PLUGGING SCHEDULE:
I-374

International Minerals \& Chemical Corp.

Potassium Prospecting Permit NM-0359163

424' North 45 degrees West from S1/4

Corner of Section 30, SW1//4 Section 30,

Township 22 South, Range 31 East. NMPM

$3340^{\prime}$

$1538^{\prime}$

Potash Core Test

Boyles Bros. Drilling Company

Date Started: $\quad 04 / 15 / 65$

Rotary Drill

Date Completed: $\quad 04 / 27 / 65$
6-1/4" Rockbit: $0-714$
3-7/8" Rockbit: $714-1149^{\prime}$
3-7/8" Corebit: $1149-1538^{\prime}$

4" Casing: 0-714'

Recovered: 0-437'

From: $1538^{\circ}$

To: $714^{\prime}$

Int: 824

Material: Cement

From: $714^{\prime}$

To: $470^{\prime}$

Int: 244'

Material: Cement

From: $470^{\prime}$

To: $20^{\prime}$

Int: $450^{\prime}$

Material: Mud

From: $20^{\circ}$

To: $0^{\prime}$

Int: $20^{\prime}$

Material: Cement

STRATIGRAPHIC SUMMARY: Rustler Formation: $360^{\circ}$

Top of Salt: $690^{\prime}$

Top of USGS 124 Bed: $1399^{\prime}$ 
BOREHOLE:

OPERATOR:

PERMIT NO.:

LOCATION:

ELEVATION:

TOTAL DEPTH:

TYPE OF WELL:

DRILLER:

DRILLING RECORD:

CASING RECORD:

PLUGGING SCHEDULE:
1-375

International Minerals \& Chemical Corp.

Potassium Prospecting Permit NM-0359161

144' South 24 degrees East from Northwest

Corner of Section 33, NW1/4 Section 33,

Township 22 South, Range 31 East. NMPM

$3390^{\prime}$

$1746^{\prime}$

Potash Core Test

Boyles Bros. Drilling Company

Date Started: $\quad 04 / 28 / 65$

Rotary Drill

Date Completed: $\quad 05 / 13 / 65$

6-1/4" Rockbit: $0-520$ '

3-7/8" Rockbit: 520-1300'

3-7/8" Corebit: $1300-1746^{\prime}$

4" Casing: 0-817'

Recovered: 0-129'

From: 1746

To: $817^{\prime}$

Int: 929'

Material: Cement

From: $817^{\prime}$

To: $510^{\prime}$

Int: $307^{\prime}$

Material: Cement

From: $510^{\prime}$

To: $20^{\prime}$

Int: $490^{\circ}$

Material: Mud

From: $20^{\prime}$

To: $0^{\prime}$

Int: $20^{\prime}$

Material: Cement

STRATIGRAPHIC SUMMARY: Rustler Formation: $470^{\prime}$

Top of Salt: $790^{\prime}$

Top of USGS 124 Bed: 1602' 


\section{COMMERCIALLY DRILLED POTASH BOREHOLE DATA BASE}

BOREHOLE:

OPERATOR:

PERMIT NO.:

LOCATION:

ELEVATION:

TOTAL DEPTH:

TYPE OF WELL:

DRILLER:

DRILLING RECORD:

CASING RECORD:

PLUGGING SCHEDULE:

\section{I-376}

International Minerals \& Chemical Corp.

Potassium Prospecting Permit NM-0384583

400' South 75 degrees East from Northwest

Corner of Section 20, NW1/4 Section 20,

Township 22 South, Range 31 East. NMPM

$3410^{\prime}$

$1702^{\prime}$

Potash Core Test

Boyles Bros. Drilling Company

Date Started: $\quad 06 / 15 / 65$

Rotary Drill

6-1/4" Rockbit: $0-840^{\circ}$

3-7/8" Rockbit: $840-1328^{\prime}$

3-7/8" Corebit: 1328-1702'

4" Casing: $0-840^{\prime}$

Recovered: $0-840$ '

From: $1702^{\circ}$

To: $800^{\prime}$

Int: $902^{\prime}$

Material: Cement

From: 800

To: $720^{\circ}$

Int: $80^{\prime}$

Material: Mud

From: $720^{\circ}$

To: $660^{\prime}$

Int: $60^{\circ}$

Material: Cement

From: $660^{\prime}$

To: $540^{\prime}$

Int: $120^{\prime}$

Material: Mud

From: $540^{\circ}$

To: $480^{\prime}$

Int: $60^{\circ}$

Material: Cement

From: $480^{\prime}$

To: $30^{\prime}$

Int: $450^{\prime}$

Material: Mud

From: $30^{\prime}$

To: $0^{\prime}$

Int: $30^{\circ}$

Material: Cement 
Top of Salt: $840^{\circ}$

Top of USGS 124 Bed: 1581' 


\section{COMMERCIALLY DRILLED POTA.SH \$OKEHOLE DATA BASE}

\begin{abstract}
BOREHOLE:
OPERATOR:

PERMIT NO.:
\end{abstract}

LOCATION:

ELEVATION:

TOTAL DEPTH:

TYPE OF WELL:

DRILLER:

DRILLING RECORD:

CASING RECORD:

PLUGGING SCHEDULE:

\section{I-377}

International Minerals \& Chemical Corp.

Potassium Prospecting Permit NM-0384584

105 ' South 48 degrees East from Northwest

Corner of Section 22, NWl/4 Section 22,

Township 22 South, Range 31 East. NMPM

$3490^{\prime}$

$1876^{\prime}$

Potash Core Test

Boyles Bros. Drilling Co.

Date Started: $06 / 26 / 65$

Rotary Drill

6-1/4" Rockbit: 0-996'

3-7/8" Rockbit: 996-1530'

4" Casing: 0-996'

Recovered: $0-996^{\prime}$

From: $1876^{\circ}$

To: 996 '

Int: $880^{\prime}$

Material: Cement

From: $996^{\circ}$

To: $925^{\prime}$

Int: $71^{\prime}$

Material: Mud

From: 925'

To: $885^{\prime}$

Int: $40^{\prime}$

Material: Cement

From: $885^{\prime}$

To: $730^{\circ}$

Int: $155^{\prime}$

Material: Mud

From: $730^{\prime}$

To: $700^{\circ}$

Int: $30^{\circ}$

Material: Cement

From: $700^{\prime}$

To: $10^{\prime}$

Int: $690^{\circ}$

Material: Mud

From: $10^{\circ}$

To: $0^{\prime}$

Int: $10^{\prime}$

Material: Cement

STRATIGRAPHIC SUMMARY: Rustler Formation: 700'

Top of Salt: 1014'

Top of USGS 124 Bed: $1770^{\prime}$ 
BOREHOLE:

OPERATOR:

PERMIT NO.:

LOCATION:

ELEVATION:

TOTAL DEPTH:

TYPE OF WELL:

DRILLER:

DRILLING RECORD:

CASING RECORD:

PLUGGING SCHEDULE:
I-383

International Minerals \& Chemical Corp.

Potassium Prospecting Permit NM-0359160

287 South 52 degrees East from W1/4

Corner of Section 1, SW1/4 Section 1,

Township 23 South, Range 30 East. NMPM

$3272^{\prime}$

$1307^{\prime}$

Potash Core Test

Boyles Bros. Drilling Company

Date Started: $\quad 07 / 19 / 65$

Date Completed: $\quad 08 / 26 / 65$

Rotary Drill

6-1/4" Rockbit: 0-481'

3-7/8" Rockbit: 481-1000'

3-7/8" Corebit: $1000-1307^{\prime}$

4" Casing: 0-481'

Recovered: 0-105'

From: $1307^{\prime}$

To: $481^{\prime}$

Int: $826^{\prime}$

Material: Cement

From: 481'

To: $320^{\circ}$

Int: $161^{\prime}$

Material: Cement

From: $320^{\prime}$

To: $210^{\prime}$

Int: $110^{\prime}$

Material: Mud

From: $210^{\prime}$

To: $150^{\prime}$

Int: $60^{\prime}$

Material: Cement

From: $150^{\prime}$

To: $15^{\prime}$

Int: $135^{\prime}$

Material: Mud

From: $15^{\prime}$

To: $0^{\prime}$

Int: $15^{\prime}$

Material: Cement

STRATIGRAPHIC SUMMARY： Rustler Formation: 160'

Top of Salt: $475^{\prime}$

Top of USGS 124 Bed: $1211^{\prime}$ 
BOREHOLE:

OPERATOR:

PERMIT NO.:

LOCATION:

ELEVATION:

TOTAL DEPTH:

TYPE OF WELL:

DRILLER:

DRILLING RECORD:

CASING RECORD:

PLUGGING SCHEDULE:

PLUGGING SCHEDULE:

. . . . .

STRATIGRAPHIC SUMMARY: Rustler Formation: $790^{\circ}$

Top of Salt: $1080^{\prime}$

Top of USGS 124 Bed: $1853^{\prime}$

I-456

$3520^{\prime}$

$1975^{\prime}$

Potash Core Test

Rotary Drill

6-1/4" Rockbit: 0-940'

3-7/8" Rockbit: 940-1580'

3-7/8" Corebit: $1580-1975$

4" Casing: Unknown

Recovered: All

From: 1975

To: $0^{\prime}$

Int: $1975^{\prime}$

Material: Cement
International Minerals \& Chemical Corp.

Potassium Prospecting Permit NM-0384584

$300^{\prime}$ from South Line and $2650^{\prime}$ from East

Line of Section 22, SW 1/4 Section 22,

Township 22 South, Range 31 East. NMPM

Boyles Bros. Drilling Company

Date Started: $\quad 06 / 22 / 76 \quad$ Date Completed: $\quad 07 / 07 / 76$ 
BOREHOLE:

OPERATOR:

PERMIT NO.:

LOCATION:

ELEVATION:

TOTAL DEPTH:

TYPE OF WELL:

DRILLER:

DRILLING RECORD:

CASING RECORD:

PLUGGing SCHEDULE:

\author{
To: $0^{\prime}$ \\ Int: $1885^{\prime}$ \\ Material: Cement
}

From: $1885^{\prime}$

$3460^{\prime}$

$1885^{\prime}$

Potash Core Test

Boyles Bros. Drilling Company

Date Started: $\quad 07 / 08 / 76$

Rotary Drill

6-1/4" Rockbit: $0-940^{\prime}$

3-7/8" Rockbit: 940-1480'

3-7/8" Corebit: $1480-1885^{\prime}$

4" Casing: 0-980'

Recovered: $0-980^{\prime}$

STRATIGRAPHIC SUMMARY: Rustler Formation: $660^{\circ}$

Top of Salt: $980^{\prime}$

Top of USGS 124 Bed: $1777^{\circ}$
Date Completed: $\quad 07 / 22 / 76$

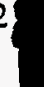

$-457$

International Minerals \& Chemical Corp.

$200^{\prime}$ from South Line and $1200^{\prime}$ from West

of Section 27, SW1/4 Section 27

Township 22 South, Range 31 East. NMPM 
BOREHOLE:

OPERATOR:

PERMIT NO.:

LOCATION:

ELEVATION:

TOTAL DEPTH:

TYPE OF WELL:

DRILLER:

CASING RECORD:

PLUGGING SCHEDULE:
DRILLING RECORD:

\section{I-458}

International Minerals \& Chemical Corp.

Potassium Prospecting Permit NM-0384584

$2500^{\prime}$ from North Line and $400^{\prime}$ from East

Line of Section 4, NE1/4 Section 4,

Township 23 South, Range 31 East. NMPM

$3410^{\prime}$

$1750^{\circ}$

Potash Core Test

Boyles Bros. Drilling Company

Date Started: $\quad 07 / 23 / 76$

Rotary Drill

6-1/4" Rockbit: 0-960'

3-7/8" Rockbit: 960-1385'

3-7/8" Corebit: $1385-1750^{\prime}$

4" Casing: Unknown

Recovered: All

From: $1750^{\prime}$

To: $0^{\prime}$

Int: $1750^{\prime}$

Material: Cement

STRATIGRAPHIC SUMMARY: Rustler Formation: 580'

Top of Salt: $970^{\prime}$

Top of USGS 124 Bed: 1623 '
Date Completed: 08/09/76 


\section{COMMERCIALLY DRILLED POTASH BOREHOLE DATA BASE}

BOREHOLE:

OPERATOR:

PERMIT NO.:

LOCATION:

ELEVATION:

TOTAL DEPTH:

TYPE OF WELL:

DRILLER:

DRILLING RECORD:

CASING RECORD:

PLUGGING SCHEDULE:

\section{I-459}

International Minerals \& Chemical Corp.

Potassium Prospecting Permit NM-0384584

$2500^{\prime}$ from North Line and 2000' from East

Line of Section 3, NE1/4 Section 3,

Township 23 South, Range 31 East. NMPM

$3385^{\prime}$

1855

Potash Core Test

Boyles Bros. Drilling Company

Date Started: $\quad 08 / 11 / 76$

Rotary Drill

6-1/4" Rockbit: $0-900$

3-7/8" Rockbit: 900-1330'

3-7/8" Corebit: $1330-1855$

4" Casing: Unknown

Recovered: All

From: 1855

To: $0^{\prime}$

Int: 1885

Material: Cement

STRATIGRAPHIC SUMMARY： Rustler Formation: $580^{\circ}$

Top of Salt: $940^{\prime}$

Top of USGS 124 Bed: 1739'
Date Completed: $\quad 08 / 24 / 76$ 


\section{BOREHOLE:}

OPERATOR:

PERMIT NO.:

LOCATION:

ELEVATION:

TOTAL DEPTH:

TYPE OF WELL:

DRILLER:

DRILLING RECORD:

CASING RECORD:

PLUGGING SCHEDULE:

\section{NFU-1}

Farmers Educational and Cooperative Union of America

Potassium Prospecting Permit NM-065503

$100^{\prime}$ from North Line and $100^{\prime}$ from West

Line of Section 9, NW 1/4 Section 9,

Township 22 South, Range 31 East. NMPM

$3422^{\prime}$

$1747^{\prime}$

Potash Core Test

Weaver Drilling Company

Date Started: $\quad 12 / 19 / 50$

Rotary Drill

6-1/4" Rockbit: 0-944'

4" Casing: $940^{\prime}$

Recovered: $675^{\prime}$

4 sacks cement at $1640^{\prime}$

7 sacks cement at $955^{\circ}$

7 sacks cement at $840^{\circ}$

8 sacks cement at $740^{\prime}$

Could not pull 4" casing, but broke circulation around it before pumping in 7 sacks at $955^{\prime}$. Shot casing at $900^{\prime}, 700^{\prime}$, and $675^{\circ}$. Recovered $675^{\prime}$ of $940^{\prime}$. Filled hole with mud and placed 4" pipe marker.

STRATIGRAPHIC SUMMARY: Rustler Formation: $635^{\prime}$

Top of Salt: $935^{\prime}$

Base of USGS 124 Bed: 1641' 


\section{COMMERCIALLY DRILLED POTASH BOREHOLE DATA BASE}

BOREHOLE:

OPERATOR:

PERMIT NO.:

LOCATION:

ELEVATION:

TOTAL DEPTH:

TYPE OF WELL:

DRILLER:

DRILLING RECORD:

CASING RECORD:

PLUGGING SCHEDULE:

\section{FC-70}

Farm Chemical Resources Development Corporation

Potassium Prospecting Permit LC-065506

$177^{\prime}$ from South Line and $177^{\prime}$ from West

Line of Section 7, NE1/4 Section 7,

Township 22 South, Range 31 East. NMPM

$3388^{\prime}$

$1603^{\prime}$

Potash Core Test

Joy Mfg. Company, Drilling Division

Date Started: $\quad 09 / 26 / 56$

Rotary Drill

6-1/4" Rockbit: 0-902'

Corebit: 902-1603'

4-1/2" Casing: 902

Recovered: 902 '

From: $1603^{\circ}$

To: $1370^{\prime}$

Int: 233'

Material: Brine \& Salt

From: $1370^{\prime}$

To: $902^{\prime}$

Int: $468^{\prime}$

Material: Cement

From: $902^{\prime}$

To: $10^{\prime}$

Int: $892^{\prime}$

Material: Mud

From: $10^{\prime}$

To: $0^{\prime}$

Int: $10^{\prime}$

Material: Cement

STRATIGRAPHIC SUMMARY: Rustler Formation: 541'

Top of Salt: $898^{\prime}$

USGS 124 Bed: $1515^{\prime}$ 
BOREHOLE:

OPERATOR:

PERMIT NO.:

LOCATION:

ELEVATION:

TOTAL DEPTH:

TYPE OF WELL:

DRILLER:

DRILLING RECORD:

CASING RECORD:

PLUGGING SCHEDULE:

\section{FC-82}

Farm Chemical Resources Development Corporation

Potassium Prospecting Permit NM-057290

$154^{\prime}$ from South Line and $37^{\prime}$ from West

Line of Section 8, Township 22 South,

Range 31 East. NMPM

$3382^{\prime}$

$1684^{\circ}$

Potash Core Test

Pennsylvania Drilling Company

Date Started: $\quad 09 / 21 / 61$

6-1/4" Rockbit: 0-922'

Corebit: $922-1684^{\prime}$

5-1/2" Casing: $922^{\prime}$

Recovered: $922^{\prime}$

From: $1684^{\prime}$

To: $922^{\prime}$

Int: 762 '

Material: Cement

From: $922^{\prime}$

To: $710^{\prime}$

Int: 212

Material: Brine \& Salt

From: $710^{\prime}$

To: $510^{\prime}$

Int: $200^{\prime}$

Material: Cement

From: $510^{\prime}$

To: $10^{\prime}$

Int: $500^{\prime}$

Material: Mud

From: $10^{r}$

To: $0^{\prime}$

Int: $10^{\prime}$

Material: Cement

STRATIGRAPHIC SUMMARY: Rustler Formation: 538'

Top of Salt: $910^{\circ}$

USGS 124 Bed: 1593'
Date Completed: 10/03/61 
BOREHOLE:

OPERATOR:

PERMIT NO.:

LOCATION:

ELEVATION:

TOTAL DEPTH:

TYPE OF WELL:

DRILLER:

DRILLING RECORD:

CASING RECORD:

PLUGGING SCHEDULE:

\section{FC-91}

Farm Chemical Resources Development Corporation

Potassium Prospecting Permit NM-075014

$200^{\prime}$ from South Line and 200' from West

Line of Section 10, SW1/4, Township 22

South, Range 31 East. NMPM

$3460^{\prime}$

$1788^{\prime}$

Potash Core Test

Pennsylvania Drilling Company

Date Started: $\quad 04 / 07 / 62$

Rotary Drill

Rockbit: 0-1070'

Corebit: $1070-1788^{\prime}$

4-1/4" Casing: $1070^{\prime}$

Recovered: $1070^{\prime}$

From: $1788^{\circ}$

To: $1060^{\prime}$

Int: $728^{\prime}$

Material: Cement

From: $1060^{\prime}$

To: $840^{\circ}$

Int: $220^{\circ}$

Material: Cuttings \& Brine

From: $840^{\prime}$

To: $800^{\prime}$

Int: $40^{\circ}$

Material: Cement

From: $800^{\prime}$

To: $10^{\prime}$

Int: $790^{\prime}$

Material: Cuttings \& Brine

From: $10^{\prime}$

To: 0 '

Int: $10^{\prime}$

Material: Cement

STRATIGRAPHIC SUMMARY: Rustler Formation: $630^{\circ}$

Top of Salt: 1070'

Base of USGS 124 Bed: Unknown
Date Completed: $\quad 04 / 17 / 62$ 
BOREHOLE:

OPERATOR:

PERMIT NO.:

LOCATION:

ELEVATION:

TOTAL DEPTH:

TYPE OF WELL:

DRILLER:

DRILLING RECORD:

CASING RECORD:

PLUGGING SCHEDULE:

\section{FC-92}

Farm Chemical Resources Development Corporation

Potassium Prospecting Permit NM-094314

$200^{\prime}$ from South Line and 200' from East

Line of Section 8, SE1/4, Township 22

South, Range 31 East. NMPM

$3420^{\prime}$

$1818^{\prime}$

Potash Core Test

Pennsylvania Drilling Company

Date Started: $\quad 04 / 21 / 62$

Rockbit: $0-1058^{\prime}$

Corebit: $1058-1818$

4-1/2" Casing: $1058^{\circ}$

Recovered: $1058^{\prime}$

From: $1818^{\prime}$

To: $945^{\circ}$

Int: $873^{\prime}$

Material: Cement

From: $945^{\prime}$

To: $850^{\circ}$

Int: $95^{\prime}$

Material: Cuttings \& Brine

From: $850^{\circ}$

To: $810^{\prime}$

Int: $40^{\prime}$

Material: Cement

From: $810^{\prime}$

To: $10^{\prime}$

Int: $800^{\prime}$

Material: Cuttings \& Brine

From: $10^{\prime}$

To: $0^{\prime}$

Int: $10^{\prime}$

Material: Cement

STRATIGRAPHIC SUMMARY: Rustler Formation: Unknown

Top of Salt: $950^{\circ}$

Base of USGS 124 Bed: Unknown
Date Completed: $\quad 05 / 03 / 62$ 


\section{COMMERCIALLY DRILLED POTASH BOREHOLE DATA BASE}

\section{BOREHOLE:}

OPERATOR:

PERMIT NO.:

LOCATION:

ELEVATION:

TOTAL DEPTH:

TYPE OF WELL:

DRILLER:

DRILLING RECORD:

CASING RECORD:

PLUGGING SCHEDULE:

\section{D-104}

Duval Sulphur \& Potash Company

Potassium Prospecting Permit PPP-058761

$2585^{\prime}$ from North Line and 1394' from East

Line of Section 24, NE1/4 Section 24,

Township 22 South, Range 30 East. NMPM

$3388^{\circ}$

$1597^{\prime}$

Potash Core Test

Weaver Drilling Company

Date Started: $\quad 09 / 25 / 52$

Rotary Drill

Date Completed: $\quad 10 / 07 / 52$

6-1/4" Rockbit: 0-759'

Corebit: $759-1597^{\circ}$

Lost Circulation: $730-747^{\prime}$

4" Casing: Unknown

Recovered: All

All casing was pulled and the following plugs run:

1) 62 sacks mixed with brine and $3 \% \mathrm{CaCl}^{\wedge} 2$ at bottom of hole.

2) 30 sacks mixed with fresh water bottomed at $660^{\circ}$.

The remainder of the hole was filled with cuttings, a four-foot marker post set, and the hole abandoned.

STRATIGRAPHIC SUMMARY: Rustler Formation: 520'

Top of Salt: $759^{\circ}$

Top of USGS 124 Bed: $1504^{\prime}$ 


\section{BOREHOLE: \\ OPERATOR: \\ PERMIT NO.:}

LOCATION:

ELEVATION:

TOTAL DEPTH:

TYPE OF WELL:

DRILLER:

DRILLING RECORD:

CASING RECORD:

PLUGGING SCHEDULE:
D-120

Duval Sulphur \& Potash Company

Potassium Prospecting Permit PPP-058761

1562' South and 1565' East of NW Corner

Section 13, NW1/4 Section 13, Township 22

South, Range 30 East. NMPM

$3338^{\prime}$

$1500^{\prime}$

Potash Core Test

Weaver Drilling Company

Date Started: $\quad 02 / 14 / 53$

Rotary Drill

6-1/4" Rockbit: 0-704'

3-13/16" Corebit: $704-1500^{\prime}$

4" Casing: 704'

Recovered: $704^{\prime}$
Date Completed: $\quad 02 / 27 / 53$

All casing was pulled and the following plugs run:

1) 62 sacks of cement mixed with brine and $3 \% \mathrm{CaCl}^{\wedge} 2$ and brine at bottom of hole.

2) 30 sacks of cement mixed with fresh water bottomed at $590^{\prime}$.

The remainder of the hole was filled with cuttings, a four-foot marker post set, and the hole abandoned.

STRATIGRAPHIC SUMMARY: Rustler Formation: $330^{\prime}$

Top of Salt: $684^{\prime}$

Top of USGS 124 Bed: $1407^{\prime}$ 
BOREHOLE:

OPERATOR:

PERMIT NO.:

LOCATION:

ELEVATION:

TOTAL DEPTH:

TYPE OF WELL:

DRILLER:

DRILLING RECORD:

CASING RECORD:

PLUGGING SCHEDULE:
D-123

Duval Sulphur \& Potash Company

Potassium Prospecting Permit LC-066112

2614' North and 277' West of Southeast

Corner of Section 34, NE1/4 Section 34,

Township 22 South, Range 31 East. NMPM

$3432^{\prime}$

$1880^{\prime}$

Potash Core Test

Weaver Drilling Company

Date Started: $\quad 07 / 19 / 53$

Rotary Drill

6-1/4". Rockbit: 0-934'

Corebit: $934-1880^{\prime}$

4" Casing: 934'

Recovered: 692'

Shot casing at $910^{\prime}$ and $850^{\prime}$. Pulled $692^{\prime}$ leaving $242^{\prime}$ in hole. The following cement plugs were run:

1) 73 sacks of cement mixed with $3 \% \mathrm{CaCl}^{\wedge} 2$ and brine at bottom of hole.

2) 45 sacks of cement mixed with fresh water bottomed at $880^{\prime}$.

The remainder of the hole was filled with cuttings, a four-foot marker post set, and the hole abandoned.

STRATIGRAPHIC SUMMARY: Rustler Formation: $670^{\circ}$

Top of Salt: $988^{\prime}$

Top of USGS 124 Bed: $1779^{\prime}$ 


\section{BOREHOLE: \\ OPERATOR: \\ PERMIT NO.:}

LOCATION:

ELEVATION:

TOTAL DEPTH:

TYPE OF WELL:

DRILLER:

DRILLING RECORD:

CASING RECORD:

PLUGGING SCHEDULE:

\section{D-160}

Duval Sulphur \& Potash Company

Potassium Prospecting Permit M-2618

$2464^{\prime}$ from South Line and $1117^{\prime}$ from West

Line of Section 36, SW1/4 Section 36,

Township 22 South, Range 30 East. NMPM

3305

1354'

Potash Core Test

Weaver Drilling Company

Date Started: $\quad 07 / 21 / 54$

Rotary Drill

6-1/4" Rockbit: 0-596'

Corebit: 596-1354'

Encountering an air blow at 860'

causing seat to leak.

4" Casing: 596'

Recovered: $596^{\circ}$

All casing was pulled and the following plugs run:

1) 58 sacks of cement mixed with brine and $3 \% . \mathrm{CaCl}^{\wedge} 2$ and brine at bottom of hole.

2) 37 sacks of cement mixed with fresh water bottomed at $445^{\prime}$.

The remainder of the hole was filled with cuttings, a four-foot marker post set, and the hole abandoned.

STRATIGRAPHIC SUMMARY: Rustler Formation: $240^{\prime}$

Top of Salt: 543

Top of USGS 124 Bed: $1301^{\prime}$ 


\section{COMMERCIALLY DRILLED POTASH BOREHOLE DATA BASE}

BOREHOLE:

OPERATOR:

PERMIT NO.:

LOCATION:

ELEVATION:

TOTAL DEPTH:

TYPE OF WELL:

DRILLER:

DRILLING RECORD:

CASING RECORD:

PLUGGING SCHEDULE:

\section{D-207}

Duval Sulphur \& Potash Company

Potassium Prospecting Permit NM-08285

$1480^{\circ}$ North and $1330^{\circ}$ East of Southwest

Corner of Section 19, SW1/4 Section 19

Township 22. South, Range 31 East. NMPM

$3406^{\prime}$

$1613^{\prime}$

Potash Core Test

Joy Drilling Company

Date Started: $\quad 06 / 16 / 58$

Rotary Drill

6-1/4" Rockbit: 0-811'

3-13/16" Corebit: 811-1613'

4" Casing: 811'

Recovered: $811^{\prime}$

All casing was pulled and the following cement plugs were run:

1) 66 sacks of cement mixed with $3 \% \mathrm{CaCl}^{\wedge} 2$ and brine at bottom of hole.

2) 25 sacks of cement mixed with fresh water bottomed at 560 '.

The remainder of the hole was filled with cuttings and soil, a four-foot marker post set, and the location left in good order.

STRATIGRAPHIC SUMMARY: Rustler Formation: $330^{\prime}$

Top of Salt: $811^{\prime}$

Top of USGS 124 Bed: $1519^{\prime}$ 
BOREHOLE:

OPERATOR:

PERMIT NO.:

LOCATION:

ELEVATION:

TOTAL DEPTH:

TYPE OF WELL:

DRILLER:

DRILLING RECORD:

CASING RECORD:

PLUGGING SCHEDULE:

STRATIGRAPHIC SUMMARY: Rustler Formation: Unknown

Top of Salt: $670^{\prime}$

Top of USGS 124 Bed: $1390^{\prime}$

D-235

3336

1506

Potash Core Test

Date Started: $\quad 10 / 11 / 77$

Rotary Drill

6-1/4" Rockbit: 0-689'

3-7/8" Corebit: 689-1506'

4" Casing: 689'

Recovered: $400^{\prime}$
Duval Sulphur \& Potash Company

Potassium Prospecting Permit LC-062188

2878' from South Line and 789' from West

Line of Section 25, NW1/4 Section 25,

Township 22 South, Range 30 East. NMPM

Pennsylvania Drilling Company

Date Completed: $\quad 11 / 03 / 77$

Encountering an air blow at $860^{\circ}$

$400^{\prime}$ of $4^{\prime \prime}$ casing was pulled. Pennsylvania Drilling Company then cemented the hole with 110 sacks of neat cement making a continuous plug from T.D. to surface. A four-foot marker post was then set and drill location was left in good order. 


\section{SUBSURFACE EXPLORATION BOREHOLE DATA BASE}

BOREHOLE:

OPERATOR:

PERMIT NO.:

LOCATION:

ELEVATION:

TOTAL DEPTH:

TYPE OF WELL:

DRILLER:

DRILLING RECORD:

CASING RECORD:
B-1

Sergent, Hauskins, \& Beckwith

Unknown

N499833.57, E666341.70

$3412.48^{\prime}$

$58.2^{\prime}$

Shallow Exploratory Drilling Converted to Observation Well 12/29/78

Sergent, Hauskins \& Beckwith

Date Started: $\quad 12 / 27 / 78$

Date Completed:

$12 / 29 / 78$

Drilling Equipment:

Truck-mounted CME-55 drill rigs powered with 6 cylinder Ford industrial engines were used in advancing test borings. The 6 cylinder engines are capable of delivering about 6,500 foot/pounds torque to the drill spindle. The spindle is advanced with twin hydraulic rams capable of exerting 12,000 pounds downward force. Drilling through soil or softer rock was performed with $61 / 2$ inch O.D., 3 $1 / 4$ inch I.D. hollow stem auger. Carbide insert teeth were used on the auger bits. Core drilling in bedrock was performed using NX size core bits with either diamond or carbide cutting faces and either air or water for drilling fluid.

PLUGGING SCHEDULE:

STRATIGRAPHIC SUMMARY: Attached 


\section{STRATIGRAPHIC SUMMARY}

BOREHOLE

B-1
Silty Sand, Sand

Caliche

Gatuna

(Santa Rosa) Sandstone
$0-9.5$

$9.5-14.5$

$14.5-38$

$38-58.2$ 


\section{SUBSURFACE EXPLORATION BOREHOLE DATA BASE}

\section{BOREHOLE: \\ OPERATOR: \\ PERMIT NO.:}

LOCATION:

ELEVATION:

TOTAL DEPTH:

TYPE OF WELL:

DRILLER:

DRILLING RECORD:

CASING RECORD:
B-1A

Sergent, Hauskins, \& Beckwith

Unknown

N499833.20, E666356.32

$3412.48^{\prime}$

$12.9^{\prime}$

Shallow Exploratory Drilling Converted to Observation Well 12/29/78

Sergent, Hauskins \& Beckwith

Date Started: $\quad 12 / 29 / 78$

Date Completed:

$12 / 29 / 78$

Drilling Equipment:

Truck-mounted CME-55 drill rigs powered with 6 cylinder Ford industrial engines were used in advancing test borings. The 6 cylinder engines are capable of delivering about 6,500 foot/pounds torque to the drill spindle. The spindle is advanced with twin hydraulic rams capable of exerting 12,000 pounds downward force. Drilling through soil or softer rock was performed with $61 / 2$ inch O.D, 3 1/4 inch I.D. hollow stem auger. Carbide insert teeth were used on the auger bits. Core drilling in bedrock was performed using NX size core bits with either diamond or carbide cutting faces and either air or water for drilling fluid.

\section{PLUGGING SCHEDULE:}

STRATIGRAPHIC SUMMARY: Attached 


\section{STRATIGRAPHIC SUMMARY}

B-1A

Sand

$0-11.2$

Caliche

11.2-12.9 
BOREHOLE:

OPERATOR:

PERMIT NO.:

LOCATION:

ELEVATION:

TOTAL DEPTH:

TYPE OF WELL:

DRILLER:

DRILLING RECORD:

CASING RECORD:

PLUGGING SCHEDULE:

STRATIGRAPHIC SUMMARY: Attached

B-2

$3411.62^{\prime}$

$33.9^{\prime}$
Sergent, Hauskins, \& Beckwith

Unknown

N499835:35, E666841.60

Shallow Exploratory Drilling

Sergent, Hauskins \& Beckwith

Date Started: $\quad 11 / 22 / 78$

Date Completed: $\quad 11 / 29 / 78$

Drilling Equipment:

Truck-mounted CME-55 drill rigs powered with 6 cylinder Ford industrial engines were used in advancing test borings. The 6 cylinder engines are capable of delivering about 6,500 foot/pounds torque to the drill spindle. The spindle is advanced with twin hydraulic rams capable of exerting 12,000 pounds downward force. Drilling through soil or softer rock was performed with $61 / 2$ inch O.D, 3 1/4 inch I.D. hollow stem auger. Carbide insert teeth were used on the auger bits. Core drilling in bedrock was performed using NX size core bits with either diamond or carbide cutting faces and either air or water for drilling fluid.

Grouted with cement. 


\section{STRATIGRAPHIC SUMMARY}

BOREHOLE

B-2

Sand

Caliche

Gatuna

Siltstone

Intercalated Sandstone and Soil
DEPTH INTERVAL IN FEET

$0-6.5$

$6.5-15.5$

$15.5-23$

23-30.6

30.6-33.9 


\section{SUBSURFACE EXPLORATION BOREHOLE DATA BASE}

BOREHOLE:

OPERATOR:

PERMIT NO.:

LOCATION:

ELEVATION:

TOTAL DEPTH:

TYPE OF WELL:

DRILLER:

DRILLING RECORD:

CASING RECORD:

PLUGGING SCHEDULE:

STRATIGRAPHIC SUMMARY: Attached

B-3

\subsection{3'}

$29^{\prime}$
Sergent, Hauskins, \& Beckwith

Unknown

N499837.13, E667341.49

Shallow Exploratory Drilling

Sergent, Hauskins \& Beckwith

Date Started: $\quad 12 / 18 / 78$

Date Completed:

$12 / 18 / 78$

Drilling Equipment:

Truck-mounted CME-55 drill rigs powered with 6 cylinder Ford industrial engines were used in advancing test borings. The 6 cylinder engines are capable of delivering about 6,500 foot/pounds torque to the drill spindle. The spindle is advanced with twin hydraulic rams capable of exerting 12,000 pounds downward force. Drilling through soil or softer rock was performed wtih $61 / 2$ inch O.D., 3 1/4 inch I.D. hollow stem auger. Carbide insert teeth were used on the auger bits. Core drilling in bedrock was performed using NX size core bits with either diamond or carbide cutting faces and either air or water for drilling fluid.

Grouted with cement. 
STRATIGRAPHIC SUMMARY

BOREHOLE

B-3

DEPTH INTERVAL IN FEET

Sand

$0-7.8$

Caliche

Gatuna

7.8-14

14-24.2

Siltstone

$24.2-25$

Sandstone

25-28

Siltstone

28-29 
BOREHOLE:

OPERATOR:

PERMIT NO.:

LOCATION:

ELEVATION:

TOTAL DEPTH:

TYPE OF WELL:

DRILLER:

DRILLING RECORD:

CASING RECORD:
B-4

Sergent, Hauskins, \& Beckwith

Unknown

N499838.91, E667841.39

3417.08

$38.8^{\prime}$

Shallow Exploratory Drilling Converted to Observation Well 12/18/78

Sergent, Hauskins \& Beckwith

Date Started: $\quad 12 / 17 / 78$

Date Completed:

$12 / 18 / 78$

Drilling Equipment:

Truck-mounted CME-55 drill rigs powered with 6 cylinder Ford industrial engines were used in advancing test borings. The 6 cylinder engines are capable of delivering about 6,500 foot/pounds torque to the drill spindle. The spindle is advanced with twin hydraulic rams capable of exerting 12,000 pounds downward force. Drilling through soil or softer rock was performed with $61 / 2$ inch O.D., 3 1/4 inch I.D. hollow stem auger. Carbide insert teeth were used on the auger bits. Core drilling in bedrock was performed using NX size core bits with either diamond or carbide cutting faces and wither air or water for drilling fluid.

PLUGGING SCHEDULE:

STRATIGRAPHIC SUMMARY: Attached 


\section{STRATIGRAPHIC SUMMARY}

B.4

Silty Sand

Caliche

$0-8.5$

Gatuna

$8.5-19$

Claystone

19-37

$37-38.8$ 


\section{SUBSURFACE EXPLORATION BOREHOLE DATA BASE}

BOREHOLE:

OPERATOR:

PERMIT NO.:

LOCATION:

ELEVATION:

TOTAL DEPTH:

TYPE OF WELL:

DRILLER:

DRILLING RECORD:

CASING RECORD:
B-4A

Sergent, Hauskins, \& Beckwith

Unknown

N499837.97,E667829.91

$3417.08^{\prime}$

$13.6^{\prime}$

Shallow Exploratory Drilling Converted to Observation Well 12/19/78

Sergent, Hauskins \& Beckwith

Date Started: $\quad 12 / 19 / 78$

Date Completed:

$12 / 19 / 78$

Drilling Equipment:

Truck-mounted CME-55 drill rigs powered with 6 cylinder Ford industrial engines were used in advancing test borings. The 6 cylinder engines are capable of delivering about 6,500 foot/pounds torque to the drill spindle. The spindle is advanced with twin hydraulic rams capable of exerting 12,000 pounds of downward force. Drilling through soil or softer rock was performed with $61 / 2$ inch O.D., 3 1/4 inch I.D. hollow stem auger. Carbide insert teeth were used on the auger bits. Core drilling in bedrock was performed using NX size core bits with either diamond or carbide cutting faces and either air or water for drilling fluid.

PLUGGING SCHEDULE:

STRATIGRAPHIC SUMMARY: Attached 


\section{STRATIGRAPHIC SUMMARY}

BOREHOLE

B-4A
ROCK UNIT

DEPTH INTERVAL IN FEET

Silty Sand

Caliche
$0-11.5$

11.5-13.6 


\section{SUBSURFACE EXPLORATION BOREHOLE DATA BASE}

BOREHOLE:

OPERATOR:

PERMIT NO.:

LOCATION:

ELEVATION:

TOTAL DEPTH:

TYPE OF WELL:

DRILLER:

DRILLING RECORD:

CASING RECORD:

PLUGGING SCHEDULE:

\section{B-5}

Sergent, Hauskins, \& Beckwith

Unknown

N499840.69, E668341.29

$3417.43^{\prime}$

$32.3^{\prime}$

Shallow Exploratory Drilling

Sergent, Hauskins \& Beckwith

Date Started: $\quad 12 / 17 / 78$

Date Completed:

$12 / 17 / 78$

Drilling Equipment:

Truck-mounted CME-55 drill rigs powered with 6 cylinder Ford industrial engines were used in advancing test borings. The 6 cylinder engines are capable of delivering about 6,500 foot/pounds torque to the drill spindle. The spindle is advanced with twin hydraulic rams capable of exerting 12,000 pounds downward force. Drilling through soil or softer rock was performed with $61 / 2$ inch O.D., 3 $1 / 4$ inch I.D. hollow stem auger. Carbide insert teeth were used on the auger bits. Core drilling in bedrock was performed using NX size core bits with either diamond or carbide cutting faces and either air or water for drilling fluid.

Grouted with cement.

STRATIGRAPHIC SUMMARY: Attached 


\section{STRATIGRAPHIC SUMMARY}

B-5

Silty Sand

$0-8.2$

Caliche

$8.2-17$

Gatuna

$17-22.4$

Sandsone

22.4-26.9

Intercalated Siltstone

26.9-32.3 
BOREHOLE:

OPERATOR:

PERMIT NO.:

LOCATION:

ELEVATION:

TOTAL DEPTH:

TYPE OF WELL:

DRILLER:

DRILLING RECORD:

CASING RECORD:

PLUGGING SCHEDULE:

STRATIGRAPHIC SUMMARY: Attached

B-6

$3422.01^{\prime}$

$26.3^{\prime}$
Sergent, Hauskins, \& Beckwith

Unknown

N499842.47, E668841.15

Shallow Exploratory Drilling

Sergent, Hauskins \& Beckwith

Date Started: $\quad 12 / 17 / 78$

Date Completed:

$12 / 17 / 78$

Drilling Equipment:

Truck-mounted CME-55 drill rigs powered with 6 cylinder Ford industrial engines were used in advancing test borings. The 6 cylinder engines are capable of delivering about 6,500 foot/pounds torque to the drill spindle. The spindle is advanced with twin hydraulic rams capable of exerting 12,000 pounds downward force. Drilling through soil or softer rock was performed with $61 / 2$ inch O.D., 3 1/4 inch I.D. hollow stem auger. Carbide insert teeth were used on the auger bits. Core drilling in bedrock was performed using $\mathrm{NX}$ size core bits with either diamond or carbide cutting faces and either air or water for drilling fluid.

Grouted with cement. 


\section{STRATIGRAPHIC SUMMARY}

B-6

Silty Sand

Caliche

$0-3.5$

3.5-10.1

Gatuna

10.1-26.3 
BOREHOLE:

OPERATOR:

PERMIT NO.:

LOCATION:

ELEVATION:

TOTAL DEPTH:

TYPE OF WELL:

DRILLER:

DRILLING RECORD:

CASING RECORD:

PLUGGING SCHEDULE:

STRATIGRAPHIC SUMMARY: Attached

B-7

$3404.19^{\prime}$

$34.9^{\prime}$
Sergent, Hauskins, \& Beckwith

Unknown

N499333.68, E666343.48

Shallow Exploratory Drilling

Sergent, Hauskins \& Beckwith

Date Started: $\quad 11 / 21 / 78$

Date Completed:

$11 / 21 / 78$

Drilling Equipment:

Truck-mounted CME-55 drill rigs powered with 6 cylinder Ford industrial engines were used in advancing test borings. The 6 cylinder engines are capable of delivering about 6,500 foot/pounds torque to the drill spindle. The spindle is advanced with twin hydraulic rams capable of exerting 12,000 pounds downward force. Drilling through soil or softer rock was performed with $61 / 2$ inch O.D., 3 1/4 inch I.D. hollow stem auger. Carbide insert teeth were used on the auger bits. Core drilling in bedrock was performed using NX size core bits with either diamond or carbide cutting faces and either air or water for drilling fluid.

Grouted with cement. 


\section{STRATIGRAPHIC SUMMARY}

BOREHOLE

B-7
ROCK UNIT

Sand

Caliche

Gatuna

Sandstone

Santa Rosa
DEPTH INTERVAL IN FEET

$0-5.5$

$5.5-20$

20-25

25-27.5

27.5-34.9 


\section{SUBSURFACE EXPLORATION BOREHOLE DATA BASE}

\section{BOREHOLE: \\ OPERATOR: \\ PERMIT NO.:}

LOCATION:

ELEVATION:

TOTAL DEPTH:

TYPE OF WELL:

DRILLER:

DRILLING RECORD:

CASING RECORD:

PLUGGING SCHEDULE:

STRATIGRAPHIC SUMMARY:
B-8

Sergent, Hauskins, \& Beckwith

Unknown

N499335.46, E666843.36

$3408.55^{\prime}$

$100^{\prime}$

Shallow Exploratory Drilling

Sergent, Hauskins \& Beckwith

Date Started: $\quad 01 / 03 / 79$

Date Completed:

$0.1 / 03 / 79$

Drilling Equipment:

Truck-mounted CME-55 drill rigs powered with 6 cylinder Ford industrial engines were used in advancing test borings. The 6 cylinder engines are capable of delivering about $6,500 \mathrm{foot} /$ pounds torque to the drill, spindle. The spindle is advanced with twin hydraulic rams capable of exerting 12,000 pounds downward force. Drilling through soil or softer rock was performed with $61 / 2$ inch O.D., 3 $1 / 4$ inch I.D. hollow stem auger. Carbide insert teeth were used on the auger bits. Core drilling in bedrock was performed using NX size core bits with either diamond or carbide cutting faces and either air or water for drilling fluid.

Grouted with cement.

Attached 


\section{STRATIGRAPHIC SUMMARY}

BOREHOLE

B-8
Sand

Caliche

Gatuna

Santa Rosa

Dewey Lake
$0-7$

$7-12$

12-35.7

$35.7-40$

$40-100$ 


\section{SUBSURFACE EXPLORATION BOREHOLE DATA BASE}

BOREHOLE:
OPERATOR:
PERMIT NO.:

LOCATION:

ELEVATION:

TOTAL DEPTH:

TYPE OF WELL:

DRILLER:

DRILLING RECORD:

CASING RECORD:

\section{B-9}

Sergent, Hauskins, \& Beckwith

Unknown

N499337.27, E667343.26

$3410.47^{\prime}$

38.3

Shallow Exploratory Drilling Sergent, Hauskins \& Beckwith

Date Started: $\quad 12 / 19 / 78$

Date Completed:

$12 / 19 / 78$

Drilling Equipment:

Truck-mounted CME-55 drill rigs powered with 6 cylinder Ford industrial engines were used in advancing test corings. The 6 cylinder engines are capable of delivering about 6,500 foot/pounds torque to the drill spindle. The spindle is advanced with twin hydraulic rams capable of exerting 12,000 pounds downward force. Drilling through soil or softer rock was performed with $61 / 2$ inch O.D., 3 $1 / 4$ inch I.D. hollow stem auger. Carbide insert teeth were used on the auger bits. Core drilling in bedrock was performed using NX size core bits with either diamond or carbide cutting faces and either air or water for drilling fluid.

PLUGGING SCHEDULE: Grouted with cement.

STRATIGRAPHIC SUMMARY: Attached 


\section{STRATIGRAPHIC SUMMARY}

BOREHOLE

B-9
ROCK UNIT

DEPTH INTERVAL IN FEET

Sand

Caliche

Gatuna

Siltstone

Sandstone
$0-8.1$

8.1-18

$18-32.1$

32.1-36

36-38:3 


\section{SUBSURFACE EXPLORATION BOREHOLE DATA BASE}

\section{BOREHOLE: \\ OPERATOR: \\ PERMIT NO.:}

LOCATION:

ELEVATION:

TOTAL DEPTH:

TYPE OF WELL:

DRILLER:

DRILLING RECORD:

CASING RECORD:
B-10

Sergent, Hauskins, \& Beckwith

Unknown

N499339.05, E6678434.11

$3413^{\prime}$

32

Shallow Exploratory Drilling Sergent, Hauskins \& Beckwith

Date Started: $\quad 12 / 19 / 78$

Date Completed:

$12 / 19 / 78$

Drilling Equipment:

Truck-mounted CME-55 drill rigs powered with 6 cylinder Ford industrial engines were used in advancing test borings. The 6 cylinder engines are capable of delivering about 6,500 foot/pounds torque to the drill spindle. The spindle is advanced with twin hydraulic rams capable of exerting 12,000 pounds downward force. Drilling through soil or softer rock was performed with $61 / 2$ inch O.D., 3 1/4 inch I.D. hollow stem auger. Carbide insert teeth were used on the auger bits. Core drilling in bedrock was performed using NX size core bits with either diamond or carbide cutting faces and either air or water for drilling fluid.

Grouted with cement.

STRATIGRAPHIC SUMMARY: Attached 
STRATIGRAPHIC SUMMARY

BOREHOLE ROCK UNIT

DEPTH INTERVAL IN FEET

B-10

Sand

Caliche

$0-10.2$

Gatuna

10.2-17

Siltstone

$17-26.5$

Sandstone

26.3-27

27-32 
BOREHOLE:

OPERATOR:

PERMIT NO.:

LOCATION:

ELEVATION:

TOTAL DEPTH:

TYPE OF WELL:

DRILLER:

DRILLING RECORD:

CASING RECORD:
B-11

Sergent, Hauskins, \& Beckwith

Unknown

N499340.83, E668343.05

$3414.30^{\prime}$

$30^{\prime}$

Shallow Exploratory Drilling

Sergent, Hauskins \& Beckwith

Date Started: $\quad 12 / 15 / 78$

Drilling Equipment:

Truck-mounted CME-55 drill rigs powered with 6 cylinder Ford industrial engines were used in advancing test borings. The 6 cylinder engines are capable of delivering about 6,500 foot/pounds torque to the drill spindle. The spindle is advanced with twin hydraulic rams capable of exerting 12,000 pounds downward force. Drilling through soil or softer rock was performed with $61 / 2$ inch O.D., 3 $1 / 4$ inch I.D. hollow stem auger. Carbide insert teeth were used on the auger bits. Core drilling in bedrock was performed using NX size core bits with either diamond or carbide cutting faces and either air or water for drilling fluid.

Grouted with cement.
PLUGGING SCHEDULE:

STRATIGRAPHIC SUMMARY: Attached 


\section{STRATIGRAPHIC SUMMARY}

BOREHOLE

B-11
ROCK UNIT

Sand

Caliche

Gatuna
DEPTH INTERVAL IN FEET

$1.2-4.1$

4.1-13.5

$13.5-30$ 


\section{SUBSURFACE EXPLORATION BOREHOLE DATA BASE}

BOREHOLE:

OPERATOR:

PERMIT NO.:

LOCATION:

ELEVATION:

TOTAL DEPTH:

TYPE OF WELL:

DRILLER:

DRILLING RECORD:

CASING RECORD:

PLUGGING SCHEDULE:

STRATIGRAPHIC SUMMARY: Attached

B-12

$3421.77^{\prime}$

$41.5^{\prime}$
Sergent, Hauskins, \& Beckwith

Unknown

N499342.61, E668842.93

Shallow Exploratory Drilling

Sergent, Hauskins \& Beckwith

Date Started: $\quad 12 / 01 / 78$

Date Completed: $\quad 12 / 01 / 78$

Drilling Equipment:

Truck-mounted CME-55 drill rigs powered with 6 cylinder Ford industrial engines were used in advancing test borings. The 6 cylinder engines are capable of delivering about 6,500 foot/pounds torque to the drill spindle. The spindle is advanced with twin hydraulic rams capable of exerting 12,000 pounds downward force. Drilling through soil or softer rock was performed with $61 / 2$ inch O.D., 3 $1 / 4$ inch I.D. hollow stem auger. Carbide insert teeth were used on the auger bits. Core drilling in bedrock was performed using NX size core bits with either diamond or carbide cutting faces and either air or water for drilling fluid.

Grouted with cement. 


\section{STRATIGRAPHIC SUMMARY}

\section{B-12}

Sand

Caliche

Gatuna

$0-6$

Sandstone

6-13

Claystone

13-20

20-26

Sandstone

26-27

Claystone

27-31.5

Sandstone

$31.5-33.5$

$33.5-38.5$

Intercalated Sandstone

$38.5-41.5$ 
BOREHOLE:

OPERATOR:

PERMIT NO.:

LOCATION:

ELEVATION:

TOTAL DEPTH:

TYPE OF WELL:

DRILLER:

DRILLING RECORD:

CASING RECORD:
B-13

Sergent, Hauskins, \& Beckwith

Unknown

N498833.82, E666345.25

$3403.91^{\prime}$

28.3

Shallow Exploratory Drilling Converted to Observation Well $12 / 16 / 78$

Sergent, Hauskins \& Beckwith

Date Started: $\quad 12 / 12 / 78$

Date Completed:

$12 / 16 / 78$

Drilling Equipment:

Truck-mounted CME-55 drill rigs powered with 6 cylinder Ford industrial engines were used in advancing test borings. The 6 cylinder engines are capable of delivering about 6,500 foot/pounds torque to the drill spindle. The spindle is advanced with twin hydraulic rams capable of exerting 12,000 pounds downward force. Drilling through soil or softer rock was performed with $61 / 2$ inch O.D., 3 1/4 inch I.D. hollow stem auger. Carbide insert teeth were used on the auger bits. Core drilling in bedrock was performed using. NX size core bits with either diamond or carbide cutting faces and either air or water for drilling fluid.

PLUGGING SCHEDULE:

STRATIGRAPHIC SUMMARY: Attached 


\section{STRATIGRAPHIC SUMMARY}

BOREHOLE

B-13
ROCK UNIT

Sand

Caliche

Gatuna

Claystone
DEPTH INTERVAL IN FEET

$0-7$

$7-12.5$

$12.5-26$

26-28.3 


\section{SUBSURFACE EXPLORATION BOREHOLE DATA BASE}

BOREHOLE:

OPERATOR:

PERMIT NO.:

LOCATION:

ELEVATION:

TOTAL DEPTH:

TYPE OF WELL:

DRILLER:

DRILLING RECORD:

CASING RECORD:

\section{B-14}

Sergent, Hauskins, \& Beckwith

Unknown

N498835.60, E666845.14

$3406.57^{\prime}$

24.5'

Shallow Exploratory Drilling

Sergent, Hauskins \& Beckwith

Date Started: $\quad 12 / 13 / 78$

Date Completed:

$12 / 13 / 78$

Drilling Equipment:

Truck-mounted CME-55 drill rigs powered with 6 cylinder Ford industrial engines were used in advancing test borings. The 6 cylinder engines are capable of delivering about 6,500 foot/pounds torque to the drill spindle. The spindle is advanced with twin hydraulic rams capable of exerting 12,000 pounds downward force. Drilling through soil or softer rock was performed with $61 / 2$ inch O.D., 3 1/4 inch I.D. hollow stem auger. Carbide insert teeth were used on the auger bits. Core drilling in bedrock was performed using NX size core bits with either diamond or carbide cutting faces and either air or water for drilling fluid.

Grouted with cement.

STRATIGRAPHIC SUMMARY: Attached 


\section{STRATIGRAPHIC SUMMARY}

BOREHOLE

B-14
ROCK UNIT

Sand

Caliche

Gatuna

Claystone
DEPTH INTERVAL IN FEET

$0-4.8$

$4.8-9.9$

$9.9-14$

$14-24.5$ 
BOREHOLE:

OPERATOR:

PERMIT NO.:

LOCATION:

ELEVATION:

TOTAL DEPTH:

TYPE OF WELL:

DRILLER:

DRILLING RECORD:

CASING RECORD:

PLUGGING SCHEDULE:

STRATIGRAPHIC SUMMARY: Attached

B-15

Unknown

$3408.64^{\prime}$

$56.8^{\prime}$
Sergent, Hauskins, \& Beckwith

N498837.40, E667345.06

Shallow Exploratory Drilling

Sergent, Hauskins \& Beckwith

Date Started: $\quad 11 / 16 / 78$

Date Completed: $\quad 11 / 17 / 78$

Drilling Equipment:

Truck-mounted CME-55 drill rigs powered with 6 cylinder Ford industrial engines were used in advancing test borings. The 6 cylinder engines are capable of delivering about 6,500 foot/pounds torque to the drill spindle. The spindle is advanced with twin hydraulic rams capable of exerting 12,000 pounds downward force. Drilling through soil or softer rock was performed with $61 / 2$ inch O.D., 3 $1 / 4$ inch I.D. hollow stem auger. Carbide insert teeth were used on the auger bits. Core drilling in bedrock was performed using NX size core bits with either diamond or carbide cutting faces and either air or water for drilling fluid.

Grouted with cement. 


\section{STRATIGRAPHIC SUMMARY}

BOREHOLE

B-15

Sand

Caliche

$0-9$

Silty Sand

9-13

13-26

Gatuna

26-51.5

Santa Rosa

Shale

$51.5-55$

$54.5-56.8$ 


\section{SUBSURFACE EXPLORATION BOREHOLE DATA BASE}

BOREHOLE:

OPERATOR:

PERMIT NO.:

LOCATION:

ELEVATION:

TOTAL DEPTH:

TYPE OF WELL:

DRILLER:

DRILLING RECORD:

CASING RECORD:

\section{B-16}

Sergent, Hauskins, \& Beckwith

Unknown

N498839.19, E667844.94

$3411.21^{\prime}$

$31^{\prime}$

Shallow Exploratory Drilling Converted to Observation Well 12/15/78

Sergent, Hauskins \& Beckwith

Date Started: $\quad 12 / 14 / 78$

Date Completed:

$12 / 15 / 78$

Drilling Equipment:

Truck-mounted CME-55 drill rigs powered with 6 cylinder Ford industrial engines were used in advancing test borings. The 6 cylinder engines are capable of delivering about 6,500 foot/pounds torque to the drill spindle. The spindle is advanced with twin hydraulic rams capable of exerting 12,000 pounds downward force. Drilling through soil or softer rock was performed with $61 / 2$ inch O.D., 3 $1 / 4$ inch I.D. hollow stem auger. Carbide insert teeth were used on the auger bits. Core drilling in bedrock was performed using NX size core bits with either diamond or carbide cutting faces and either air or water for drilling fluid.

PLUGGING SCHEDULE:

STRATIGRAPHIC SUMMARY： Attached 


\section{STRATIGRAPHIC SUMMARY}

BOREHOLE

B-16
ROCK UNIT

Sand

Caliche

Gatuna
DEPTH INTERVAL IN FEET

$0-7$

7-15

15-31 


\section{BOREHOLE: OPERATOR: \\ PERMIT NO.:}

LOCATION:

ELEVATION:

TOTAL DEPTH:

TYPE OF WELL:

DRILLER:

DRILLING RECORD:

CASING RECORD:

PLUGGING SCHEDULE:
B-17

Sergent, Hauskins, \& Beckwith Unknown

N498840.97, E668343.86

$3413.26^{\prime}$

$25.8^{\prime}$

Shallow Exploratory Drilling

Sergent, Hauskins \& Beckwith

Date Started: $\quad 12 / 13 / 78$

Date Completed:

$12 / 13 / 78$

Drilling Equipment:

Truck-mounted CME-55 drill rigs powered with 6 cylinder Ford industrial engines were used in advancing test borings. The 6 cylinder engines are capable of delivering about 6,500 foot/pounds torque to the drill spindle. The spindle is advanced with twin hydraulic rams capable of exerting 12,000 pounds downward force. Drilling through soil or softer rock was performed with $61 / 2$ inch O.D., 3 1/4 inch I.D. hollow stem auger. Carbide insert teeth were used on the auger bits. Core drilling in bedrock was performed using NX size core bits with either diamond or carbide cutting faces and either air or water for drilling fluid.

STRATIGRAPHIC SUMMARY: Attached 


\section{STRATIGRAPHIC SUMMARY}

BOREHOLE

B-17
ROCK UNIT

Silty Sand

Caliche

Gatuna
DEPTH INTERVAL IN FEET

$0-5.1$

5.1-10.5

$10.5-25.8$ 
BOREHOLE:

OPERATOR:

PERMIT NO.:

LOCATION:

ELEVATION:

TOTAL DEPTH:

TYPE OF WELL:

DRILLER:

DRILLING RECORD:

CASING RECORD:

\author{
B-18 \\ Sergent, Hauskins, \& Beckwith \\ Unknown
}

N498842.75, E668844.75

$3419.32^{\prime}$

$33.3^{\prime}$

Shallow Exploratory Drilling Converted to Observation Well 12/15/78

Sergent, Hauskins \& Beckwith

Date Started: $\quad 12 / 14 / 78$

Date Completed:

$12 / 15 / 78$

Drilling Equipment:

Truck-mounted CME-55 drill rigs powered with 6 cylinder Ford industrial engines were used in advancing test borings. The 6 cylinder engines are capable of delivering about 6,500 foot/pounds torque to the drill spindle. The spindle is advanced with twin hydraulic rams capable of exerting 12,000 pounds downward force. Drilling through soil or softer rock was performed with $61 / 2$ inch O.D., 3 1/4 inch I.D. hollow stem auger. Carbide insert teeth were used on the auger bits. Core drilling in bedrock was performed using NX size core bits with either diamond or carbide cutting faces and either air or water for drilling fluid.

PLUGGING SCHEDULE:

STRATIGRAPHIC SUMMARY: Attached 


\section{STRATIGRAPHIC SUMMARY}

BOREHOLE

B-18
ROCK UNIT

Sand

Caliche

Gatuna
DEPTH INTERVAL IN FEET

$0-7.1$

7.1-15

$15-33.3$ 


\section{SUBSURFACE EXPLORATION BOREHOLE DATA BASE}

BOREHOLE:

OPERATOR:

PERMIT NO.:

LOCATION:

ELEVATION:

TOTAL DEPTH:

TYPE OF WELL:

DRILLER:

DRILLING RECORD:

CASING RECORD:

PLUGGING SCHEDULE:

STRATIGRAPHIC SUMMARY： Attached

B-19

Unknown

$3399.84^{\prime}$

$38.8^{\prime}$
Sergent, Hauskins, \& Beckwith

N498333.94, E666347.03

Shallow Exploratory Drilling

Sergent, Hauskins \& Beckwith

Date Started: $\quad 11 / 30 / 78$

Date Completed: $\quad 11 / 30 / 78$

Drilling Equipment:

Truck-mounted CME-55 drill rigs powered with 6 cylinder Ford industrial engines were used in advancing test borings. The 6 cylinder engines are capable of delivering about 6,500 foot/pounds torque to the drill spindle. The spindle is advanced with twin hydraulic rams capable of exerting 12,000 pounds downward force. Drilling through soil or softer rock was performed with $61 / 2$ inch O.D., 3 1/4 inch I.D. hollow stenr auger. Carbide insert teeth were used on the auger bits. Core drilling in bedrock was performed using NX size core bits with either diamond or carbide cutting faces and either air or water for drilling fluid.

Grouted with cement. 


\section{STRATIGRAPHIC SUMMARY}

B-19

Sand

0-8.5

Caliche

$8.5-18.5$

Gatuna

$18.5-21.1$

Sandstone

$21.1-38.8$ 


\section{SUBSURFACE EXPLORATION BOREHOLE DATA BASE}

BOREHOLE:

OPERATOR:

PERMIT NO.:

LOCATION:

ELEVATION:

TOTAL DEPTH:

TYPE OF WELL:

DRILLER:

DRILLING RECORD:

CASING RECORD:
B-20

Sergent, Hauskins, \& Beckwith

Unknown

N498335.72, E666846.91

$3403.53^{r}$

14 '

Shallow Exploratory Drilling Converted to Observation Well 12/16/78

Sergent, Hauskins \& Beckwith

Date Started: 12/12/78 Date Completed: 12/16/78

Drilling Equipment:

Truck-mounted CME-55 drill rigs powered with 6 cylinder Ford industrial engines were used in advancing test borings. The 6 cylinder engines are capable of delivering about 6,500 foot/pounds torque to the drill spindle. The spindle is advanced.with twin hydraulic rams capable of exerting 12,000 pounds downward force. Drilling through soil or softer rock was performed with $61 / 2$ inch O.D., 3 $1 / 4$ inch I.D. hollow stem auger. Carbide insert teeth were used on the auger bits. Core drilling in bedrock was performed using NX size core bits with either diamond or carbide cutting faces and either air or water for drilling fluid.

PLUGGING SCHEDULE:

STRATIGRAPHIC SUMMARY: Attached 


\section{STRATIGRAPHIC SUMMARY}

BOREHOLE

B-20
ROCK UNIT

DEPTH INTERVAL IN FEET

Sand

Caliche
0-10

10-14 


\section{SUBSURFACE EXPLORATION BOREHOLE DATA BASE}

BOREHOLE:
OPERATOR:
PERMIT NO.:

LOCATION:

ELEVATION:

TOTAL DEPTH:

TYPE OF WELL:

DRILLER:

DRILLING RECORD:

CASING RECORD:

PLUGGING SCHEDULE:

STRATIGRAPHIC SUMMARY: Attached

\section{B-20A}

Unknown

$3403.53^{\prime}$

$34.2^{\prime}$

\section{STRATIGRAPHIC SUMMARY: Attohed}

Sergent, Hauskins, \& Beckwith

N498341.34, E666847.10

Shallow Exploratory Drilling Converted to Observation Well 12/16/78

Sergent, Hauskins \& Beckwith

Date Started: $\quad 12 / 12 / 78$

Date Completed: $\quad 12 / 16 / 78$

Drilling Equipment:

Truck-mounted CME-55 drill rigs powered with 6 cylinder Ford industrial engines were used in advancing test borings. The 6 cylinder engines are capable of delivering about 6,500 foot/pounds torque to the drill spindle. The spindle is advanced with twin hydraulic rams capable of exerting 12,000 pounds downward force. Drilling through soil or softer rock was performed with $61 / 2$ inch O.D., 3 1/4 inch I.D. hollow stem auger. Carbide insert teeth were used on the auger bits. Core drilling in bedrock was performed using NX size core bits with either diamond or carbide cutting faces and either air or water for drilling. 


\section{STRATIGRAPHIC SUMMARY}

BOREHOLE

B-20A
ROCK UNIT

DEPTH INTERVAL IN FEET

Sand

Caliche

Gatuna
$0-13.9$

13.9-19

$19-34.2$ 
BOREHOLE:

OPERATOR:

PERMIT NO.:

LOCATION:

ELEVATION:

TOTAL DEPTH:

TYPE OF WELL:

DRILLER:

DRILLING RECORD:

CASING RECORD:

PLUGGING SCHEDULE:
B-21

Sergent, Hauskins, \& Beckwith

Unknown

N498337.50, E667346.75

$3404.55^{\prime}$

$40.4^{\prime}$

Shallow Exploratory Drilling

Sergent, Hauskins \& Beckwith

Date Started: $\quad 11 / 17 / 78$

Date Completed: $\quad 11 / 17 / 78$

Drilling Equipment:

Truck-mounted CME-55 drill rigs powered with 6 cylinder Ford industrial engines were used in advancing test borings. The 6 cylinder engines are capable of delivering about $6,500 \mathrm{foot} /$ pounds torque to the drill spindle. The spindle is advanced with twin hydraulic rams capable of exerting 12,000 pounds downward force. Drilling through soil or softer rock was performed with $61 / 2$ inch O.D., 3 $1 / 4$ inch I.D. hollow stem auger. Carbide insert teeth were used on the auger bits. Core drilling in bedrock was performed using NX size core bits with either diamond or carbide cutting faces and either air or water for drilling fluid.

STRATIGRAPHIC SUMMARY: Attached 
STRATIGRAPHIC SUMMARY

BOREHOLE

B-21

Sand

Caliche

Gatuna

Sandstone
DEPTH INTERVAL IN FEET

$0-9.5$

9.5-20

20-32.5

$32.5-40.4$ 
BOREHOLE:

OPERATOR:

PERMIT NO.:

LOCATION:

ELEVATION:

TOTAL DEPTH:

TYPE OF WELL:

DRILLER:

DRILLING RECORD:

CASING RECORD:

PLUGGING SCHEDULE:

STRATIGRAPHIC SUMMARY:
B-22

Sergent, Hauskins, \& Beckwith

Unknown

N498339.28, E667846.73

$3406.85^{\prime}$

$27.8^{\prime}$

Shallow Exploratory Drilling

Sergent, Hauskins \& Beckwith

Date Started: $\quad 12 / 11 / 78$

Date Completed: $\quad 12 / 11 / 78$

Drilling Equipment:

Truck-mounted CME-55 drill rigs powered with 6 cylinder Ford industrial engines were used in advancing test borings. The 6 cylinder engines are capable of delivering about 6,500 foot/pounds torque to the drill spindle. The spindle is advanced with twin hydraulic rams capable of exerting 12,000 pounds downward force. Drilling through soil or softer rock was performed with $61 / 2$ inch O.D., 3 1/4 inch I.D. hollow stem auger. Carbide insert teeth were used on the auger bits. Core drilling in bedrock was performed using NX size core bits with either diamond or carbide cutting faces and either air or water for drilling fluid.

Grouted with cement.

Attached 


\section{STRATIGRAPHIC SUMMARY}

BOREHOLE

B-22
ROCK UNIT

Sand

Caliche

Gatuna
DEPTH INTERVAL IN FEET

$0-7.5$

$7.5-13$

$13-27.8$ 


\section{SUBSURFACE EXPLORATION BOREHOLE DATA BASE}

BOREHOLE:

OPERATOR:

PERMIT NO.:

LOCATION:

ELEVATION:

TOTAL DEPTH:

TYPE OF WELL:

DRILLER:

DRILLING RECORD:

CASING RECORD:

PLUGGING SCHEDULE:

STRATIGRAPHIC SUMMARY: Attached

\section{B-23}

Unknown

$3412.07^{\prime}$

$40.5^{\prime}$
Sergent, Hauskins, \& Beckwith

N498341.06, E668346.63

Shallow Exploratory Drilling

Sergent, Hauskins \& Beckwith

Date Started: $\quad 12 / 06 / 78$

Date Completed:

$12 / 06 / 78$

Drilling Equipment:

Truck-mounted CME-55 drill rigs powered with 6 cylinder Ford industrial engines were used in advancing test borings. The 6 cylinder engines are capable of delivering about 6,500 foot/pounds torque to the drill spindle. The spindle is advanced with twin hydraulic rams capable of exerting 12,000 pounds downward force. Drilling through soil or softer rock was performed with $61 / 2$ inch O.D., 3 1/4 inch I.D. hollow stem auger. Carbide insert teeth were used on the auger bits. Core drilling in bedrock was performed using NX size core bits with either diamond or carbide cutting faces and either air or water for drilling fluid.

Grouted with cement. 


\section{STRATIGRAPHIC SUMMARY}

B-23

Sand

Caliche

0-6

Gatuna

6-14.3

$14.3-40.5$ 
BOREHOLE:

OPERATOR:

PERMIT NO.:

LOCATION:

ELEVATION:

TOTAL DEPTH:

TYPE OF WELL:

DRILLER:

DRILLING RECORD:

CASING RECORD:

PLUGGING SCHEDULE:

STRATIGRAPHIC SUMMARY: Attached

\section{B-24}

Unknown

$3417.87^{\prime}$

29.3'
Sergent, Hauskins, \& Beckwith

N498342.84, E668846.53

Shallow Exploratory Drilling

Sergent, Hauskins \& Beckwith

Date Started: $\quad 12 / 07 / 78$

Date Completed:

$12 / 07 / 78$

Drilling Equipment:

Truck-mounted CME-55 drill rigs powered with 6 cylinder Ford industrial engines were used in advancing test borings. The 6 cylinder engines are capable of delivering about 6,500 foot/pounds torque to the drill spindle. The spindle is advanced with twin hydraulic rams capable of exerting 12,000 pounds downward force. Drilling through soil or softer rock was performed with $61 / 2$ inch O.D., 3 1/4 inch I.D. hollow stem auger. Carbide insert teeth were used on the auger bits. Core drilling in bedrock was performed using NX size core bits with either diamond or carbide cutting faces and either air or water for drilling fluid.

Grouted with cement. 


\section{STRATIGRAPHIC SUMMARY}

B-24

Sand

$0-5.5$

Caliche

5.5-12

Gatuna

$12=29.3$ 


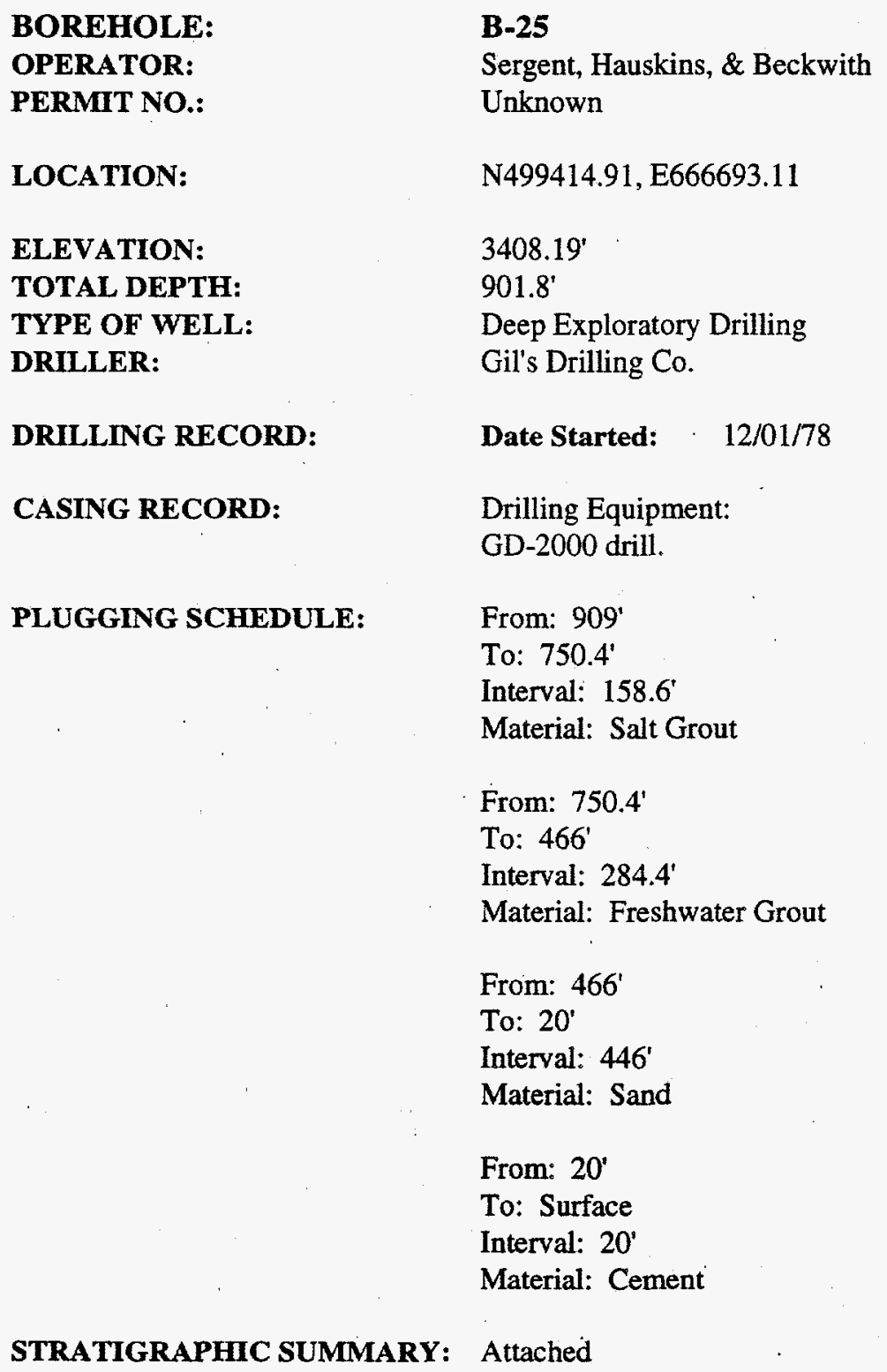

\author{
B-25
Sergent, Hauskins, \& Beckwith \\ Unknown
}

$901.8^{\prime}$
Deep Exploratory Drilling

Gil's Drilling Co.

Drilling Equipment:

GD-2000 drill.

N499414.91, E666693.11

Date Started: 12/01/78

Date Completed: $\quad 01 / 18 / 79$

\section{Date Completed:}




\section{STRATIGRAPHIC SUMMARY}

\section{B-25}

Sand

$0-10$

Caliche

10-14

Gatuna

14-34.7

Santa Rosa

$34.7-44.8$

Dewey Lake

44.8-533

Rustler Formation

533-842.9

Magenta Member

$592.7-617$

Culebra Member

704.1-728

Salado Formation

$842.9-901.8$ 
BOREHOLE:

OPERATOR:

PERMIT NO.:

LOCATION:

ELEVATION:

TOTAL DEPTH:

TYPE OF WELL:

DRILLER:

DRILLING RECORD:

CASING RECORD:

PLUGGING SCHEDULE:

STRATIGRAPHIC SUMMARY:

\section{B-26}

Sergent, Hauskins, \& Beckwith

Unknown

N499600.58, E666892.43

$3410.19^{\prime}$

27.5

Shallow Exploratory Drilling

Sergent, Hauskins \& Beckwith

Date Started: $\quad 01 / 03 / 79$

Date Completed:

01/03/79

Drilling Equipment:

Truck-mounted CME-55 drill rigs powered with 6 cylinder Ford industrial engines were used in advancing test borings. The 6 cylinder engines are capable of delivering about 6,500 foot/pounds torque to the drill spindle. The spindle is advanced with twin hydraulic rams capable of exerting 12,000 pounds downward force. Drilling through soil or softer rock was performed with $61 / 2$ inch O.D., 3 1/4 inch I.D. hollow stem auger. Carbide insert teeth were used on the auger bits. Core drilling in bedrock was performed using NX size core bits with either diamond or carbide cutting faces and either air or water for drilling fluid.

Grouted with cement.

Attached 


\section{STRATIGRAPHIC SUMMARY}

BOREHOLE

B-26
ROCK UNIT

DEPTH INTERVAL IN FEET

Sand

Caliche

Gatuna

Siltstone
$0-5$

5-11

11-23

23-27.5 
BOREHOLE:

OPERATOR:

PERMIT NO.:

LOCATION:

ELEVATION:

TOTAL DEPTH:

TYPE OF WELL:

DRILLER:

DRILLING RECORD:

CASING RECORD:

PLUGGING SCHEDULE:

STRATIGRAPHIC SUMMARY: Attached

B-27

Unknown

$3400.20^{\prime}$

$25.8^{\prime}$
Sergent, Hauskins, \& Beckwith

N498982.08, E665854.70

Shallow Exploratory Drilling

Sergent, Hauskins \& Beckwith

Date Started: $\quad 01 / 11 / 79$

Date Completed: $\quad 01 / 11 / 79$

Drilling Equipment:

Truck-mounted CME-55 drill rigs powered with 6 cylinder Ford industrial engines were used in advancing test borings. The 6 cylinder engines are capable of delivering about 6,500 foot/pounds torque to the drill spindle. The spindle is advanced with twin hydraulic rams capable of exerting 12,000 pounds downward force. Drilling through soil or softer rock was performed with $61 / 2$ inch O.D., 3 $1 / 4$ inch I.D. hollow stem auger. Carbide insert teeth were used on the auger bits. Core drilling in bedrock was performed using NX size core bits with either diamond or carbide cutting faces and either air or water for drilling fluid.

Grouted with cement. 


\section{STRATIGRAPHIC SUMMARY}

DEPTH INTERVAL IN FEET

\section{B-27}

Sand

Caliche

Gatuna

Sandstone

Claystone

Sandstone

Intercalated Sandstone

4-11

$11-22$

22-22.5

22.5-23

23-24

24-25.8 


\section{SUBSURFACE EXPLORATION BOREHOLE DATA BASE}

BOREHOLE:

OPERATOR:

PERMIT NO.:

LOCATION:

ELEVATION:

TOTAL DEPTH:

TYPE OF WELL:

DRILLER:

DRILLING RECORD:

CASING RECORD:

PLUGGING SCHEDULE:

STRATIGRAPHIC SUMMARY:

\section{B-28}

Sergent, Hauskins, \& Beckwith

Unknown

N499295.02, E666718.53

3408.17

$27^{\prime}$

Shallow Exploratory Drilling

Sergent, Hauskins \& Beckwith

Date Started: $\quad 01 / 04 / 79$

Date Completed:

$01 / 04 / 79$

Drilling Equipment:

Truck-mounted CME-55 drill rigs powered with 6 cylinder Ford industrial engines were used in advancing test borings. The 6 cylinder engines are capable of delivering about 6,500 foot/pounds torque to the drill spindle. The spindle is advanced with twin hydraulic rams capable of exerting 12,000 pounds downward force. Drilling through soil or softer rock was performed with $61 / 2$ inch O.D., 3 $1 / 4$ inch I.D. hollow stem auger. Carbide insert teeth were used on the auger bits. Core drilling in bedrock was performed using NX size core bits with either diamond or carbide cutting faces and either air or water for drilling fluid.

Grouted with cement.

Attached 
STRATIGRAPHIC SUMMARY

BOREHOLE

B-28
ROCK UNIT

Sand

Caliche

Gatuna

Sandstone
DEPTH INTERVAL IN FEET

0-6

6-12

12-21

21-27 
BOREHOLE:

OPERATOR:

PERMIT NO.:

LOCATION:

ELEVATION:

TOTAL DEPTH:

TYPE OF WELL:

DRILLER:

DRILLING RECORD:

CASING RECORD:

PLUGGING SCHEDULE:

STRATIGRAPHIC SUMMARY: Attached

B-29

$3410.87^{\prime}$

$28.7^{\prime}$
Sergent, Hauskins, \& Beckwith

Unknown

N499397.21, E667253.07

Shallow Exploratory Drilling

Sergent, Hauskins \& Beckwith

Date Started: $\quad 12 / 30 / 78$

Date Completed: $\quad 12 / 30 / 78$

Drilling Equipment:

Truck-mounted CME-55 drill rigs powered with 6 cylinder Ford industrial engines were used in advancing test borings. The 6 cylinder engines are capable of delivering about 6,500 foot/pounds torque to the drill spindle. The spindle is advanced with twin hydraulic rams capable of exerting 12,000 pounds downward force. Drilling through soil or softer rock was performed with $61 / 2$ inch O.D., 3 1/4 inch I.D. hollow stem auger. Carbide insert teeth were used on the auger bits. Core drilling in bedrock was performed using NX size core bits with either diamond or carbide cutting faces and either air or water for drilling fluid.

Grouted with cement. 


\section{STRATIGRAPHIC SUMMARY}

BOREHOLE

B-29
ROCK UNIT

Sand

Gatuna
DEPTH INTERVAL IN FEET

$0-13$

13-28.7 
BOREHOLE:

OPERATOR:

PERMIT NO.:

LOCATION:

ELEVATION:

TOTAL DEPTH:

TYPE OF WELL:

DRILLER:

DRILLING RECORD:

CASING RECORD:

PLUGGING SCHEDULE:

STRATIGRAPHIC SUMMARY:
B-30

Sergent, Hauskins, \& Beckwith

Unknown

N499185.81, E666933.86

$3408.75^{\prime}$

27.8

Shallow Exploratory Drilling

Sergent, Hauskins \& Beckwith

Date Started: $\quad 12 / 28 / 78$

Date Completed:

$12 / 28 / 78$

Drilling Equipment:

Truck-mounted CME-55 drill rigs powered with 6 cylinder Ford industrial engines were used in advancing test borings. The 6 cylinder engines are capable of delivering about 6,500 foot/pounds torque to the drill spindle. The spindle is advanced with twin hydraulic rams capable of exerting 12,000 pounds downward force. Drilling through soil or softer rock was performed with $61 / 2$ inch O.D., 3 $1 / 4$ inch I.D. hollow stem auger. Carbide insert teeth were used on the auger bits. Core drilling in bedrock was performed using NX size core bits with either diamond or carbide cutting faces and either air or water for drilling fluid.

Grouted with cement.

Attached 
STRATIGRAPHIC SUMMARY

BOREHOLE

B-30
ROCK UNIT

Sand

Caliche

Gatuna

Sandstone
DEPTH INTERVAL IN FEET

$$
0-7.5
$$

7.5-11.5

$11.5-17.6$

$17.6-27.8$ 


\section{SUBSURFACE EXPLORATION BOREHOLE DATA BASE}

\section{BOREHOLE: \\ OPERATOR: \\ PERMIT NO.:}

LOCATION:

ELEVATION:

TOTAL DEPTH:

TYPE OF WELL:

DRILLER:

DRILLING RECORD:

CASING RECORD:

PLUGGING SCHEDULE:

STRATIGRAPHIC SUMMARY:

\section{B-31}

Sergent, Hauskins, \& Beckwith

Unknown

N499206.60, E667143.76

3410.27

$30.5^{\prime}$

Shallow Exploratory Drilling

Sergent, Hauskins \& Beckwith

Date Started: $\quad 12 / 29 / / 78$

Date Completed:

$12 / 29 / 78$

Drilling Equipment:

Truck-mounted CME-55 drill rigs powered with 6 cylinder Ford industrial engines were used in advancing test borings. The 6 cylinder engines are capable of delivering about 6,500 foot/pounds torque to the drill spindle. The spindle is advanced with twin hydraulic rams capable of exerting 12,000 pounds downward force. Drilling through soil or softer rock was performed with $61 / 2$ inch O.D., 3 1/4 inch I.D. hollow stem auger. Carbide insert teeth were used on the auger bits. Core drilling in bedrock was performed using NX size core bits with either diamond or carbide cutting faces and either air or water for drilling fluid.

Grouted with cement.

Attached 


\section{STRATIGRAPHIC SUMMARY}

BOREHOLE

B-31
ROCK UNIT

Sand

Caliche

Gatuna
DEPTH INTERVAL IN FEET

$0-7.8$

7.8-13.4

$13.4-30.5$ 
BOREHOLE:

OPERATOR:

PERMIT NO.:

LOCATION:

ELEVATION:

TOTAL DEPTH:

TYPE OF WELL:

DRILLER:

DRILLING RECORD:

CASING RECORD:
B-32

Sergent, Hauskins, \& Beckwith

Unknown

N499187.96, E667523.76

$3410.89^{\prime}$

$100^{\prime}$

Shallow Exploratory Drilling

Sergent, Hauskins \& Beckwith

Date Started: $\quad 01 / 07 / 79$

Date Completed:

$01 / 07 / 79$

Drilling Equipment:

Truck-mounted CME-55 drill rigs powered with 6 cylinder Ford industrial engines were used in advancing test borings. The 6 cylinder engines are capable of delivering about 6,500 foot/pounds torque to the drill spindle. The spindle is advanced with twin hydraulic rams capable of exerting 12,000 pounds downward force. Drilling through soil or softer rock was performed with $61 / 2$ inch O.D., 3 1/4 inch I.D. hollow stem auger. Carbide insert teeth were used on the auger bits. Core drilling in bedrock was performed using NX size core bits with either diamond or carbide cutting faces and either air or water for drilling fluid.

PLUGGING SCHEDULE: Grouted with cement.

STRATIGRAPHIC SUMMARY: Attached 


\section{STRATIGRAPHIC SUMMARY}

BOREHOLE

B-32

Sand

Caliche

Gatuna

Siltstone \& Claystone

Santa Rosa

Dewey Lake
DEPTH INTERVAL IN FEET

$0-12.5$

12.5-19.5

$19.5-26.5$

$26.5-43$

43-53

$53-100$ 
BOREHOLE:

OPERATOR:

PERMIT NO.:

LOCATION:

ELEVATION:

TOTAL DEPTH:

TYPE OF WELL:

DRILLER:

DRILLING RECORD:

CASING RECORD:

\section{B-33}

Sergent, Hauskins, \& Beckwith

Unknown

N498874.29, E666470.03

$3404.05^{\prime}$

30.7

Shallow Exploratory Drilling

Sergent, Hauskins \& Beckwith

Date Started: $\quad 12 / 29 / 78$

Date Completed:

$12 / 29 / 78$

Drilling Equipment:

Truck-mounted CME-55 drill rigs powered with 6 cylinder Ford industrial engines were used in advancing test borings. The 6 cylinder engines are capable of delivering about 6,500 foot/pounds torque to the drill spindle. The spindle is advanced with twin hydraulic rams capable of exerting 12,000 pounds downward force. Drilling through soil or softer rock was performed with $61 / 2$ inch O.D., 3 1/4 inch I.D. hollow stem auger. Carbide insert teeth were used on the auger bits. Core drilling in bedrock was performed using NX size core bits with either diamond or carbide curring faces and either air or water for drilling fluid.

Grouted with cement.

PLUGGING SCHEDULE:

STRATIGRAPHIC SUMMARY: Attached 


\section{STRATIGRAPHIC SUMMARY}

B-33

Sand

$0-6.7$

Caliche

$6.7-15.8$

Gatuna

$15.8-19$

Sandstone

19-24

Intercalated Siltstone \& Mudstone

Sandstone

$24-29.8$

29.8-30.7 
BOREHOLE:

OPERATOR:

PERMIT NO.:

LOCATION:

ELEVATION:

TOTAL DEPTH:

TYPE OF WELL:

DRILLER:

DRILLING RECORD:

CASING RECORD:
B-34

Sergent, Hauskins, \& Beckwith

Unknown

N498205.49, E666762.36

$3401.97^{\prime}$

$100^{\prime}$

Shallow Exploratory Drilling

Sergent, Hauskins \& Beckwith

Date Started: 01/09/79

Date Completed:

$01 / 09 / 79$

Drilling Equipment

Truck-mounted CME-55 drill rigs powered with 6 cylinder Ford industrial engines were used in advancing test borings. The 6 cylinder engines are capable of delivering about 6,500 foot/pounds torque to the drill spindle. The spindle is advanced with twin hydraulic rams capable of exerting 12,000 pounds downward force. Drilling through soil or softer rock was performed with $61 / 2$ inch O.D., 3. 1/4 inch I.D. hollow stem auger. Carbide insert teeth were used on the auger bits. Core drilling in bedrock was performed using NX size core bits with either diamond or carbide cutting faces and either air or water for drilling fluid.
PLUGGING SCHEDULE:
Grouted with cement.

STRATIGRAPHIC SUMMARY: Attached 


\section{STRATIGRAPHIC SUMMARY}

BOREHOLE

B-34

\begin{abstract}
Sand
\end{abstract}
Caliche

Gatura

Siltstone \& Claystone
DEPTH INTERVAL IN FEET

$0-9.1$

$9.1-14.8$

$14.8-40$

40-100 


\section{SUBSURFACE EXPLORATION BOREHOLE DATA BASE}

BOREHOLE:

OPERATOR:

PERMIT NO.:

LOCATION:

ELEVATION:

TOTAL DEPTH:

TYPE OF WELL:

DRILLER:

DRILLING RECORD:

CASING RECORD:

PLUGGING SCHEDULE:

STRATIGRAPHIC SUMMARY:
B-35

Sergent, Hauskins, \& Beckwith

Unknown

N498326.97, E667196.82

3402.55

32

Shallow Exploratory Drilling

Sergent, Hauskins \& Beckwith

Date Started: $\quad 01 / 05 / 79$

Date Completed:

$01 / 05 / 79$

Drilling Equipment:

Truck-mounted CME-55 drill rigs powered with 6 cylinder Ford industrial engines were used in advancing test borings. The 6 cylinder engines are capable of delivering about 6,500 foot/pounds torque to the drill spindle. The spindle is advanced with twin hydraulic rams capable of exerting 12,000 pounds downward force. Drilling through soil or softer rock was performed with $61 / 2$ inch O.D., 3 1/4 inch I.D. hollow stem auger. Carbide insert teeth were used on the auger bits. Core drilling in bedrock was performed using NX size core bits with either diamond or carbide cutting faces and either air or water for drilling fluid.

Grouted with cement.

Attached 


\section{STRATIGRAPHIC SUMMARY}

BOREHOLE

B-35

Sand

Caliche

Gatuna

Sandstone
DEPTH INTERVAL IN FEET

0-7

7-12

12-23

23-32 


\section{SUBSURFACE EXPLORATION BOREHOLE DATA BASE}

BOREHOLE:

OPERATOR:

PERMIT NO.:

LOCATION:

ELEVATION:

TOTAL DEPTH:

TYPE OF WELL:

DRILLER:

DRILLING RECORD:

CASING RECORD:

PLUGGING SCHEDULE:

STRATIGRAPHIC SUMMARY: Attached

\section{B-36}

3422.03

27.8
Sergent, Hauskins, \& Beckwith

Unknown

N499293.25, E669018.07

Shallow Exploratory Drilling

Sergent, Hauskins \& Beckwith

Date Started: $\quad 01 / 07 / 79$

Date Completed:

$01 / 07 / 79$

Drilling Equipment:

Truck-mounted CME-55 drill rigs powered with 6 cylinder Ford industrial engines were used in advancing test borings. The 6 cylinder engines are capable of delivering about 6,500 foot/pounds torque to the drill spindle. The spindle is advanced with twin hydraulic rams capable of exerting 12,000 pounds downward force. Drilling through soil or softer rock was performed with $61 / 2$ inch O.D., 3 1/4 inch I.D. hollow stem auger. Carbide insert teeth were used on the auger bits. Core drilling in bedrock was performed using NX size core bits with either diamond or carbide cutting faces and either air or water for drilling fluid.

Grouted with cement. 


\section{STRATIGRAPHIC SUMMARY}

BOREHOLE

B-36
ROCK UNIT

DEPTH INTERVAL IN FEET

Sand

Caliche

Gatuna

Siltstone
$0-5.9$

5.9-13.2

$13.2-25$

$25-27.8$ 


\section{SUBSURFACE EXPLORATION BOREHOLE DATA BASE}

BOREHOLE:

OPERATOR:

PERMIT NO.:

LOCATION:

ELEVATION:

TOTAL DEPTH:

TYPE OF WELL:

DRILLER:

DRILLING RECORD:

CASING RECORD:
B-37

Sergent, Hauskins, \& Beckwith

Unknown

N499297.91, E670352.77

$3438.91^{\prime}$

$27.5^{\prime}$

Shallow Exploratory Drilling

Sergent, Hauskins \& Beckwith

\section{Date Started: $\quad 01 / 09 / 79$}

Date Completed:

$01 / 09 / 79$

Drilling Equipment:

Truck-mounted CME-55 drill rigs powered with 6 cylinder Ford industrial engines were used in advancing test borings. The 6 cylinder engines are capable of delivering about 6,500 foot/pounds torque to the drill spindle. The spindle is advanced with twin hydraulic rams capable of exerting 12,000 pounds downward force. Drilling through soil or softer rock was performed with $61 / 2$ inch O.D., 3 $1 / 4$ inch I.D. hollow stem auger. Carbide insert teeth were used on the auger bits. Core drilling in bedrock was performed using NX size core bits with either diamond or carbide cutting faces and either air or water for drilling fluid.

\section{PLUGGING SCHEDULE: Grouted with cement.}

STRATIGRAPHIC SUMMARY: Attached 
STRATIGRAPHIC SUMMARY

BOREHOLE

B-37
ROCK UNIT

Sand

Caliche

Gatuna

Siltstone

Sandstone
DEPTH INTERVAL IN FEET

$0-5.5$

$5.5-12.5$

$12.5-20$

$20-20.3$

20.3-27.5 


\section{SUBSURFACE EXPLORATION BOREHOLE DATA BASE}

\section{BOREHOLE: \\ OPERATOR: \\ PERMIT NO.:}

LOCATION:

ELEVATION:

TOTAL DEPTH:

TYPE OF WELL:

DRILLER:

DRILLING RECORD:

CASING RECORD:

\author{
B-37A \\ Sergent, Hạskins, \& Beckwith \\ Unknown
}

$\mathrm{N} 499260.53, \mathrm{E} 669370.39$

$3426.68^{\prime}$

$22.4^{\prime}$

Shallow Exploratory Drilling

Sergent, Hauskins \& Beckwith

Date Started: 01/08/79

Date Completed:

$01 / 08 / 79$

Drilling Equipment:

Truck-mounted CME-55 drill rigs powered with 6 cylinder Ford industrial engines were used in advancing test borings. The 6 cylinder engines are capable of delivering about 6,500 foot/pounds torque to the drill spindle. The spindle is advanced with twin hydraulic rams capable of exerting 12,000 pounds downward force. Drilling through soil or softer rock was performed with $61 / 2$ inch O.D., 3 1/4 inch I.D. hollow stem auger. Carbide insert teeth were used on the auger bits. Core drilling in bedrock was performed using NX size core bits with either diamond or carbide cutting faces and either air or water for drilling fluid.

Grouted with cement.

STRATIGRAPHIC SUMMARY: Attached 


\section{STRATIGRAPHIC SUMMARY}

BOREHOLE

B-37A
ROCK UNIT

Sand

Caliche

Gatuna
DEPTH INTERVAL IN FEET

$0-5.5$

5.5-13.5

$13.5-22.4$ 


\section{SUBSURFACE EXPLORATION BOREHOLE DATA BASE}

BOREHOLE:

OPERATOR:

PERMIT NO.:

LOCATION:

ELEVATION:

TOTAL DEPTH:

TYPE OF WELL:

DRILLER:

DRILLING RECORD:

CASING RECORD:

\author{
B-38 \\ Sergent, Hauskins, \& Beckwith \\ Unknown
}

N499155.65, E669683.42

$3429.88^{\prime}$

$50^{\prime}$

Shallow Exploratory Drilling Converted to Observation Well 1/23/79

Sergent, Hauskins \& Beckwith

Date Started: $\quad 01 / 11 / 79$

Date Completed:

$01 / 23 / 79$

Drilling Equipment:

Truck-mounted CME-5,5 drill rigs powered with 6 cylinder Ford industrial engines were used in advancing test borings. The 6 cylinder engines are capable of delivering about 6,500 foot/pounds torque to the drill spindle. The spindle is advanced with twin hydraulic rams capable of exerting 12,000 pounds downward force. Drilling through soil or softer rock was performed with $61 / 2$ inch O.D., 3 1/4 inch I.D. hollow stem auger. Carbide insert teeth were used on the auger bits. Core drilling in bedrock was performed using NX size core bits with either diamond or carbide cutting faces and either air or water for drilling fluid.

PLUGGING SCHEDULE:

STRATIGRAPHIC SUMMARY： Attached 


\section{STRATIGRAPHIC SUMMARY}

BOREHOLE

B-38
ROCK UNIT

Sand

Caliche

Gatuna

Sandstone

Santa Rosa

Dewey Lake
DEPTH INTERVAL IN FEET

$0-5.5$

$5.5-14$

14-20.5

$20.5-40.2$

$40.2-43.7$

$43.7-50$ 


\section{SUBSURFACE EXPLORATION BOREHOLE DATA BASE}

BOREHOLE:

OPERATOR:

PERMIT NO.:

LOCATION:

ELEVATION:

TOTAL DEPTH:

TYPE OF WELL:

DRILLER:

DRILLING RECORD:

CASING RECORD:

PLUGGING SCHEDULE:

STRATIGRAPHIC SUMMARY: Attached

\section{B-39}

$3422.08^{\prime}$

27.6
Sergent, Hauskins, \& Beckwith

Unknown

N499018.31, E669019.04

Shallow Exploratory Drilling

Sergent, Hauskins \& Beckwith

Date Started: $\quad 01 / 06 / 79$

Date Completed:

$01 / 07 / 79$

Drilling Equipment:

Truck-mounted CME-55 drill rigs powered with 6 cylinder Ford industrial engines were used in advancing test borings. The 6 cylinder engines are capable of delivering about 6,500 foot/pounds torque to the drill spindle. The spindle is advanced with twin hydraulic rams capable of exerting 12,000 pounds downward force. Drilling through soil or softer rock was performed with $61 / 2$ inch O.D., 3 1/4 inch I.D. hollow stem auger. Carbide insert teeth were used on the auger bits. Core drilling in bedrock was performed using NX size core bits with either diamond or carbide cutting faces and either air or water for drilling fluid.

Grouted with cement. 


\section{STRATIGRAPHIC SUMMARY}

BOREHOLE

B-39

Sand

Caliche

Gatuna
DEPTH INTERVAL IN FEET

$0-7$

7-13

$13-27.6$ 


\author{
BOREHOLE: \\ OPERATOR: \\ PERMIT NO.:
}

LOCATION:

ELEVATION:

TOTAL DEPTH:

TYPE OF WELL:

DRILLER:

DRILLING RECORD:

CASING RECORD:

\author{
B-40 \\ Sergent, Hauskins, \& Beckwith \\ Unknown
}

N499023.04, E670353.77

$3438.48^{\prime}$

$27.9^{\prime}$

Shallow Exploratory Drilling

Sergent, Hauskins \& Beckwith

Date Started: $\quad 01 / 10 / 79$

Drilling Equipment:

Truck-mounted CME-55 drill rigs powered with 6 cylinder Ford industrial engines were used in advancing test borings. The 6 cylinder engines are capable of delivering about 6,500 foot/pounds torque to the drill spindle. The spindle is advanced with twin hydraulic rams capable of exerting 12,000 pounds downward force. Drilling through soil or softer rock was performed with $61 / 2$ inch O.D., 3 1/4 inch I.D. hollow stem auger. Carbide insert teeth were used on the auger bits. Core drilling in bedrock was performed using NX size core bits with either diamond or carbide cutting faces and either air or water for drilling fluid.

PLUGGING SCHEDULE: $\quad$ Grouted with cement.

STRATIGRAPHIC SUMMARY: Attached 
STRATIGRAPHIC SUMMARY

BOREHOLE

B-40 ROCK UNIT

DEPTH INTERVAL IN FEET

Sand

Caliche

Gatuna

Sandstone
$0-7.6$

7.6-13

13-19

$19-27.9$ 


\section{SUBSURFACE EXPLORATION BOREHOLE DATA BASE}

BOREHOLE:

OPERATOR:

PERMIT NO:

LOCATION:

ELEVATION:

TOTAL DEPTH:

TYPE OF WELL:

DRILLER:

DRILLING RECORD:

CASING RECORD:

PLUGGING SCHEDULE:

STRATIGRAPHIC SUMMARY: Attached

B-41

$3407.86^{\circ}$

$100^{\prime}$
Sergent, Hauskins, \& Beckwith

Unknown

N499335.51, E666858.36

Shallow Exploratory Drilling

Sergent, Hauskins \& Beckwith

Date Started: $\quad 01 / 05 / 79$

Date Completed:

$01 / 05 / 79$

Drilling Equipment:

Truck-mounted CME-55 drill rigs powered with 6 cylinder Ford industrial engines were used in advancing test borings. The 6 cylinder engines are capable of delivering about 6,500 foot/pounds torque to the drill spindle. The spindle is advanced with twin hydraulic rams capable of exerting 12,000 pounds downward force. Drilling through soil or softer rock was performed with $61 / 2$ inch O.D., 3 1/4 inch I.D. hollow stem auger. Carbide insert teeth were used on the auger bits. Core drilling in bedrock was performed using NX size core bits with either diamond or carbide cutting faces and either air or water for drilling fluid.

Grouted with cement. 


\section{STRATIGRAPHIC SUMMARY}

BOREHOLE

\section{B-41}

ROCK UNIT

Sand

Caliche

Gatuna

Santa Rosa

Dewey Lake
DEPTH INTERVAL IN FEET

0-6

6-15

$15-42$

$42-48$

48-100 


\section{SUBSURFACE EXPLORATION BOREHOLE DATA BASE}

\section{BOREHOLE: \\ OPERATOR: \\ PERMIT NO.:}

LOCATION:

ELEVATION:

TOTAL DEPTH:

TYPE OF WELL:

DRILLER:

DRILLING RECORD:

CASING RECORD:

\section{B-42}

Sergent, Hauskins, \& Beckwith Unknown

N499335.57, E666873.35

$3408.99^{\prime}$

$100^{\prime}$

Shallow Exploratory Drilling

Sergent, Hauskins \& Beckwith

Date Started: $\quad 01 / 06 / 79$

Date Completed:

$01 / 06 / 79$

Drilling Equipment:

Truck-mounted CME-55 drill rigs powered with 6 cylinder Ford industrial engines were used in advancing test borings. The 6 cylinder engines are capable of delivering about 6,500 foot/pounds torque to the drill spindle. The spindle is advanced with twin hydraulic rams capable of exerting 12,000 pounds downward force. Drilling through soil or softer rock was performed with $61 / 2$ inch O.D., 3 1/4 inch I.D. hollow stem auger. Carbide insert teeth were used on the auger bits. Core drilling in bedrock was performed using NX size core bits with either diamond or carbide cutting faces and either air or water for drilling fluid.

Grouted with cement.

STRATIGRAPHIC SUMMARY： Attached 


\section{STRATIGRAPHIC SUMMARY}

BOREHOLE

B-42

Sand

Caliche

Gatuna

Santa Rosa

Dewey Lake
DEPTH INTERVAL IN FEET

0-6

6-14

14-42

$42-48$

$48-100$ 


\section{SUBSURFACE EXPLORATION BOREHOLE DATA BASE}

BOREHOLE:

OPERATOR:

PERMIT NO.:

LOCATION:

ELEVATION:

TOTAL DEPTH:

TYPE OF WELL:

DRILLER:

DRILLING RECORD:

CASING RECORD:

PLUGGING SCHEDULE:

STRATIGRAPHIC SUMMARY: Attached

B-43

Unknown

$3410.75^{\prime}$

$100^{\prime}$
Sergent, Hauskins, \& Beckwith

N499202.96, E667523.70

Shallow Exploratory Drilling

Sergent, Hauskins \& Beckwith

Date Started: $\quad 01 / 06 / 79$

Date Completed: $\quad 01 / 06 / 79$

Drilling Equipment:

Truck-mounted CME-55 drill rigs powered with 6 cylinder Ford industrial engines were used in advancing test borings. The 6 cylinder engines are capable of delivering about 6,500 foot/pounds torque to the drill spindle. The spindle is advanced with twin hydraulic rams capable of exerting 12,000 pounds downward force. Drilling through soil or softer rock was performed with $61 / 2$ inch O.D., 3 $1 / 4$ inch I.D. hollow stem auger. Carbide insert teeth were used on the auger bits. Core drilling in bedrock was performed using NX size core bits with either diamond or carbide cutting faces and either air or water for drilling fluid.

Grouted with cement. 


\section{STRATIGRAPHIC SUMMARY}

BOREHOLE

B-43

Sand

Caliche

Gatuna

Santa Rosa

Dewey Lake
DEPTH INTERVAL IN FEET

$0-12$

12-20

20-43

43-53

53-100 


\section{SUBSURFACE EXPLORATION BOREHOLE DATA BASE}

BOREHOLE:

OPERATOR:

PERMIT NO.:

LOCATION:

ELEVATION:

TOTAL DEPTH:

TYPE OF WELL:

DRILLER:

DRILLING RECORD:

CASING RECORD:

PLUGGING SCHEDULE:

STRATIGRAPHIC SUMMARY:
B-44

Sergent, Hauskins, \& Beckwith

Unknown

N499172.96, E667523.82

$3411.48^{\prime}$

$100^{\circ}$

Shallow Exploratory Drilling

Sergent, Hauskins \& Beckwith

Date Started: $\quad 01 / 08 / 79$

Date Completed:

$01 / 08 / 79$

Drilling Equipment:

Truck-mounted CME-55 drill rigs powered with 6 cylinder Ford industrial engines were used in advancing test borings. The 6 cylinder engines are capable of delivering about 6,500 foot/pounds torque to the drill spindle. The spindle is advanced with twin hydraulic rams capable of exerting 12,000 pounds downward force. Drilling through soil or softer rock was performed with $61 / 2$ inch O.D., 3 1/4 inch I.D. hollow stem auger. Carbide insert teeth were used on the auger bits. Core drilling in bedrock was performed using NX size core bits with either diamond or carbide cutting faces and either air or water for drilling fluid.

Grouted with cement.

Attached 


\section{STRATIGRAPHIC SUMMARY}

BOREHOLE

B-44
ROCK UNIT

Sand

Caliche

Gatuna

Santa Rosa

Dewey Lake
DEPTH INTERVAL IN FEET

0-16

16-21

21-38

38-52

52-100 


\section{SUBSURFACE EXPLORATION BOREHOLE DATA BASE}

\section{BOREHOLE: \\ OPERATOR: \\ PERMIT NO.:}

LOCATION:

ELEVATION:

TOTAL DEPTH:

TYPE OF WELL:

DRILLER:

DRILLING RECORD:

CASING RECORD:

\section{B-45}

Sergent, Hauskins, \& Beckwith

Unknown

N498205.44, E666747.36

$3401.51^{\prime}$

$100^{\prime}$

Shallow Exploratory Drilling

Sergent, Hauskins \& Beckwith

Date Started: $\quad 01 / 10 / 79$

Date Completed:

$01 / 17 / 79$

Drilling Equipment:

Truck-mounted CME-55 drill rigs powered with 6 cylinder Ford industrial engines were used in advancing test borings. The 6 cylinder engines are capable of delivering about 6,500 foot/pounds torque to the drill spindle. The spindle is advanced with twin hydraulic rams capable of exerting 12,000 pounds downward force. Drilling through soil or softer rock was performed with $61 / 2$ inch O.D., 3 $1 / 4$ inch I.D. hollow stem auger. Carbide insert teeth were used on the auger bits. Core drilling in bedrock was performed using NX size core bits with either diamond or carbide cutting faces and either air or water for drilling fluid.

PLUGGING SCHEDULE: Grouted with cement.

STRATIGRAPHIC SUMMARY: Attached 


\section{STRATIGRAPHIC SUMMARY}

BOREHOLE

\section{B-45}

ROCK UNIT

DEPTH INTERVAL IN FEET

Sand

Caliche

Gatuna

Dewey Lake

Sandstone

Siltstone
$0-7.5$

$7.5-15.3$

15.3-31.3

$31.3-44.2$

$44.2-44.7$

$44.7-100$ 


\section{SUBSURFACE EXPLORATION BOREHOLE DATA BASE}

BOREHOLE:

OPERATOR:

PERMIT NO.:

LOCATION:

ELEVATION:

TOTAL DEPTH:

TYPE OF WELL:

DRILLER:

DRILLING RECORD:

CASING RECORD:

PLUGGING SCHEDULE:

STRATIGRAPHIC SUMMARY: Attached

B-46

$3401.98^{\prime}$

$100^{\prime}$
Sergent, Hauskins, \& Beckwith

Unknown

N498205.54, E666777.36

Shallow Exploratory Drilling

Sergent, Hauskins \& Beckwith

Date Started: $\quad 01 / 08 / 79$

Date Completed: $\quad 01 / 08 / 79$

Drilling Equipment:

Truck-mounted CME-55 drill rigs powered with 6 cylinder Ford industrial engines were used in advancing test borings. The 6 cylinder engines are capable of .delivering about 6,500 foot/pounds torque to the drill spindle. The spindle is advanced with twin hydraulic rams capable of exerting 12,000 pounds downward force. Drilling through soil or softer rock was performed with $61 / 2$ inch O.D., 3 1/4 inch I.D. hollow stem auger. Carbide insert teeth were used on the auger bits. Core drilling in bedrock was performed using NX size core bits with either diamond or carbide cutting faces and either air or water for drilling fluid.

Grouted with cement. 


\section{STRATIGRAPHIC SUMMARY}

BOREHOLE

B-46
ROCK UNIT

Sand

Caliche

Gatuna

Santa Rosa .

Dewey Lake
DEPTH INTERVAL IN FEET

0-8

8-13

13-41

$41-48$

$48-100$ 


\section{SUBSURFACE EXPLORATION BOREHOLE DATA BASE}

BOREHOLE:

OPERATOR:

PERMIT NO.:

LOCATION:

ELEVATION:

TOTAL DEPTH:

TYPE OF WELL:

DRILLER:

DRILLING RECORD:

CASING RECORD:

\section{B-47}

Sergent, Hauskins, \& Beckwith

Unknown

N499206.77, E667193.82

$3409.49^{\prime}$

$17.5^{\prime}$

Shallow Exploratory Drilling

Sergent, Hauskins \& Beckwith

Date Started: $\quad 01 / 25 / 79$

Date Completed:

$01 / 25 / 79$

Drilling Equipment:

Truck-mounted CME-55 drill rigs powered with 6 cylinder Ford industrial engines were used in advancing test borings. The 6 cylinder engines are capable of delivering about 6,500 foot/pounds torque to the drill spindle. The spindle is advanced with twin hydraulic rams capable of exerting 12,000 pounds downward force. Drilling through soil or softer rock was performed with $61 / 2$ inch O.D., 3 1/4 inch I.D. hollow stem auger. Carbide insert teeth were used on the auger bits. Core drilling in bedrock was performed using NX size core bits with either diamond or carbide cutting faces and either air or water for drilling fluid.
PLUGGING SCHEDULE:

STRATIGRAPHIC SUMMARY:
Grouted with cement. 


\section{STRATIGRAPHIC SUMMARY}

B-47

Sand

$0-8.9$

Caliche

$8.9-15$

Gatuna

$15-17.5$ 
BOREHOLE:

OPERATOR:

PERMIT NO.:

LOCATION:

ELEVATION:

TOTAL DEPTH:

TYPE OF WELL:

DRILLER:

DRILLING RECORD:

CASING RECORD:

PLUGGING SCHEDULE:

STRATIGRAPHIC SUMMARY: Attached

B-48

Unknown

$3412.12^{\prime}$

$15.7^{\prime}$
Sergent, Hauskins, \& Beckwith

N498839.26, E667854.93

Shallow Exploratory Drilling

Sergent, Hauskins \& Beckwith

Date Started: $\quad 01 / 24 / 79$

Date Completed:

$01 / 24 / 79$

Drilling Equipment:

Truck-mounted CME-55 drill rigs powered with 6 cylinder Ford industrial engines were used in advancing test borings. The 6 cylinder engines are capable of delivering about 6,500 foot/pounds torque to the drill spindle. The spindle is advanced with twin hydraulic rams capable of exerting 12,000 pounds downward force. Drilling through soil or softer rock was performed with $61 / 2$ inch O.D., 3 $1 / 4$ inch I.D. hollow stem auger. Carbide insert teeth were used on the auger bits. Core drilling in bedrock was performed using NX size core bits with either diamond or carbide cutting faces and either air or water for drilling fluid.

Grouted with cement. 


\section{STRATIGRAPHIC SUMMARY}

BOREHOLE

B-48

Sand

Caliche
DEPTH INTERVAL IN FEET

0-9

9-15.7 


\section{SUBSURFACE EXPLORATION BOREHOLE DATA BASE}

BOREHOLE:

OPERATOR:

PERMIT NO.:

LOCATION:

ELEVATION:

TOTAL DEPTH:

TYPE OF WELL:

DRILLER:

DRILLING RECORD:

CASING RECORD:

PLUGGING SCHEDULE:

STRATIGRAPHIC SUMMARY: Attached

$3409.07^{\prime}$

$19.2^{\prime}$

\author{
B-49 \\ Sergent, Hauskins, \& Beckwith \\ Unknown
}

N498937.22, E667294.71

Shallow Exploratory Drilling

Sergent, Hauskins \& Beckwith

Date Started: $\quad 01 / 24 / 79$

Date Completed:

$01 / 24 / 79$

Drilling Equipment:

Truck-mounted CME-55 drill rigs powered with 6 cylinder Ford industrial engines were used in advancing test borings. The 6 cylinder engines are capable of delivering about 6,500 foot/pounds torque to the drill spindle. The spindle is advanced with twin hydraulic rams capable of exerting 12,000 pounds downward force. Drilling through soil or softer rock was performed with $61 / 2$ inch O.D., 3 $1 / 4$ inch I.D. hollow stem auger. Carbide insert teeth were used on the auger bits. Core drilling in bedrock was performed using NX size core bits with either diamond or carbide cutting faces and either air or water for drilling fluid.

Grouted with cement. 


\section{STRATIGRAPHIC SUMMARY}

BOREHOLE

B-49
ROCK UNIT

DEPTH INTERVAL IN FEET

Sand

Caliche

Silty Sand

Gatuna
$0-8.5$

$8.5-10.5$

$10.5-15$

$15-19.2$ 


\section{SUBSURFACE EXPLORATION BOREHOLE DATA BASE}

BOREHOLE:

OPERATOR:

PERMIT NO.:

LOCATION:

ELEVATION:

TOTAL DEPTH:

TYPE OF WELL:

DRILLER:

DRILLING RECORD:

CASING RECORD:

PLUGGING SCHEDULE:

STRATIGRAPHIC SUMMARY: Attached

\section{B-50}

$3405.65^{\prime}$

$23.7^{\prime}$
Sergent, Hauskins, \& Beckwith

Unknown

N498487.09, E667236.23

Shallow Exploratory Drilling

Sergent, Hauskins \& Beckwith

Date Started: $\quad 01 / 26 / 79$

Date Completed:

$01 / 26 / 79$

Drilling Equipment:

Truck-mounted CME-55 drill rigs powered with 6 cylinder Ford industrial engines were used in advancing test borings. The 6 cylinder engines are capable of delivering about 6,500 foot/pounds torque to the drill spindle. The spindle is advanced with twin hydraulic rams capable of exerting 12,000 pounds downward force. Drilling through soil or softer rock was performed with $61 / 2$ inch O.D., 3 $1 / 4$ inch I.D. hollow stem auger. Carbide insert teeth were used on the auger bits. Core drilling in bedrock was performed using NX size core bits with either diamond or carbide cutting faces and either air or water for drilling fluid.

Grouted with cement. 
STRATIGRAPHIC SUMMARY

B-50

Sand

$0-9.5$

Caliche

9.5-15

Gatuna

$15-23.7$ 


\section{SUBSURFACE EXPLORATION BOREHOLE DATA BASE}

\section{BOREHOLE: \\ OPERATOR: \\ PERMIT NO.:}

LOCATION:

ELEVATION:

TOTAL DEPTH:

TYPE OF WELL:

DRILLER:

DRILLING RECORD:

CASING RECORD:

PLUGGING SCHEDULE:

\section{B-51}

Sergent, Hauskins, \& Beckwith

Unknown

N498337.48, E667341.75

$3404.74^{\prime}$

15.2'

Shallow Exploratory Drilling

Sergent, Hauskins \& Beckwith

Date Started: $\quad 01 / 25 / 79$

Date Completed:

$01 / 25 / 79$

Drilling Equipment:

Truck-mounted CME-55 drill rigs powered with 6 cylinder Ford industrial engines were used in advancing test borings. The 6 cylinder engines are capable of delivering about 6,500 foot/pounds torque to the drill spindle. The spindle is advanced with twin hydraulic rams capable of exerting 12,000 pounds downward force. Drilling through soil or softer rock was performed with $61 / 2$ inch O.D., 3 $1 / 4$ inch I.D. hollow stem auger. Carbide insert teeth were used on the auger bits. Core drilling in bedrock was performed using NX size core bits with either diamond or carbide cutting faces and either air or water for drilling fluid.

Grouted with cement.

STRATIGRAPHIC SUMMARY: Attached 


\section{STRATIGRAPHIC SUMMARY}

BOREHOLE

B-51
ROCK UNIT

Sand

Caliche

Gatuna
DEPTH INTERVAL IN FEET

$0-7.5$

7.5-14.5

14.5-15.2 


\section{SUBSURFACE EXPLORATION BOREHOLE DATA BASE}

BOREHOLE:

OPERATOR:

PERMIT NO.:

LOCATION:

ELEVATION:

TOTAL DEPTH:

TYPE OF WELL:

DRILLER:

DRILLING RECORD:

CASING RECORD:

PLUGGING SCHEDULE:

STRATIGRAPHIC SUMMARY: Attached

B-52

$3385.48^{\prime}$

$30.0^{\prime}$
Sergent, Hauskins, \& Beckwith

Unknown

N496981.21, E665461.91

Shallow Exploratory Drilling

Sergent, Hauskins \& Beckwith

Date Started: $\quad 01 / 26 / 79$

Date Completed:

$01 / 26 / 79$

Drilling Equipment:

Truck-mounted CME-55 drill rigs powered with 6 cylinder Ford industrial engines were used in advancing test borings. The 6 cylinder engines are capable of . delivering about 6,500 foot/pounds torque to the drill spindle. The spindle is advanced with twin hydraulic rams capable of exerting 12,000 pounds downward force. Drilling through soil or softer rock was performed with $61 / 2$ inch O.D., 3 1/4 inch I.D. hollow stem auger. Carbide insert teeth were used on the auger bits. Core drilling in bedrock was performed using NX size core bits with either diamond or carbide cutting faces and either air or water for drilling fluid.

Grouted with cement. 
STRATIGRAPHIC SUMMARY

BOREHOLE

B-52
ROCK UNIT

DEPTH INTERVAL IN FEET

Sand

Caliche

Gatuna

Dewey Lake
$0-3.1$

3.1-8.5

8.5-19

$19-30$ 
BOREHOLE:

OPERATOR:

PERMIT NO.:

LOCATION:

ELEVATION:

TOTAL DEPTH:

TYPE OF WELL:

DRILLER:

DRILLING RECORD:

CASING RECORD:

PLUGGING SCHEDULE:

STRATIGRAPHIC SUMMARY:
B-53

Sergent, Hauskins, \& Beckwith

Unknown

N496651.28, E665463.05

$3386.65^{\prime}$

$30.2^{\prime}$

Shallow Exploratory Drilling

Sergent, Hauskins \& Beckwith

Date Started: $\quad 01 / 30 / 79$

Date Completed:

$01 / 30 / 79$

Drilling Equipment:

Truck-mounted CME-55 drill rigs powered with 6 cylinder Ford industrial engines were used in advancing test borings. The 6 cylinder engines are capable of delivering about 6,500 foot/pounds torque to the drill spindle. The spindle is advanced with twin hydraulic rams capable of exerting 12,000 pounds downward force. Drilling through soil or softer rock was performed with $61 / 2$ inch O.D., 3 $1 / 4$ inch I.D. hollow stem auger. Carbide insert teeth were used on the auger bits. Core drilling in bedrock was performed using NX size core bits with either diamond or carbide cutting faces and either air or water for drilling fluid.

Grouted with cement.

Attached 


\section{STRATIGRAPHIC SUMMARY}

BOREHOLE

B-53
ROCK UNIT

Sand

Caliche

Gatuna

Dewey Lake
DEPTH INTERVAL IN FEET

$0-8.5$

8.5-15.7

$15.7-23.7$

23.7-30.2 


\section{SUBSURFACE EXPLORATION BOREHOLE DATA BASE}

BOREHOLE:

OPERATOR:

PERMIT NO.:

LOCATION:

ELEVATION:

TOTAL DEPTH:

TYPE OF WELL:

DRILLER:

DRILLING RECORD:

CASING RECORD:
B-54

Sergent, Hauskins, \& Beckwith

Unknown

N499386.52, E666651.92

$3408.60^{\prime}$

$210^{\prime}$

Shallow Exploratory Drilling. Converted to Observation Well 02/14/79

Sergent, Hauskins \& Beckwith

Date Started: $\quad 02 / 13 / 79 \quad$ Date Completed: $\quad 02 / 14 / 79$

Drilling Equipment:

Truck-mounted CME-55 drill rigs powered with 6 cylinder Ford industrial engines were used in advancing test borings. The 6 cylinder engines are capable of delivering about 6,500 foot/pounds torque to the drill spindle. The spindle is advanced with twin hydraulic rams capable of exerting 12,000 pounds downward force. Drilling through soil or softer rock was performed with $61 / 2$ inch O.D., 3 $1 / 4$ inch I.D. hollow stem auger. Carbide insert teeth were used on the auger bits. Core drilling in bedrock was performed using NX size core bits with either diamond or carbide cutting faces and either air or water for drilling fluid.

PLUGGING SCHEDULE:

STRATIGRAPHIC SUMMARY: Attached 


\section{STRATIGRAPHIC SUMMARY}

BOREHOLE

B-54

$\begin{array}{lc}\text { Sand } & 0-6 \\ \text { Caliche } & 6-12 \\ \text { Gatuna } & 12-25 \\ \text { Santa Rosa } & 25-30 \\ \text { Dewey Lake } & 30-210\end{array}$


BOREHOLE:

OPERATOR:

PERMIT NO.:

LOCATION:

ELEVATION:

TOTAL DEPTH:

TYPE OF WELL:

DRILLER:

DRILLING RECORD:

CASING RECORD:

PLUGGING SCHEDULE:
B-301

Sergent, Hauskins, \& Beckwith

Unknown

North Access Road

Not Recorded

$39.7^{\prime}$

Shallow Exploratory Drilling

Sergent, Hauskins \& Beckwith

Date Started: ， 02/08/79

Date Completed:

$02 / 08 / 79$

Drilling Equipment:

Truck-mounted CME-55 drill rigs powered with 6 cylinder Ford industrial engines were used in advancing test borings. The 6 cylinder engines are capable of delivering about 6,500 foot/pounds torque to the drill spindle. The spindle is advanced with twin hydraulic rams capable of exerting 12,000 pounds downward force. Drilling through soil or softer rock was performed with $61 / 2$ inch O.D., 3 1/4 inch I.D. hollow stem auger. Carbide insert teeth were used on the auger bits. Core drilling in bedrock was performed using NX size core bits with either diamond or carbide cutting faces and either air or water for drilling fluid.

Backfilled with native drilled material

STRATIGRAPHIC SUMMARY: Attached 


\section{STRATIGRAPHIC SUMMARY}

BOREHOLE

B-301
ROCK UNIT

DEPTH INTERVAL IN FEET

Sand

Caliche

Dewey Lake
0-1

1-24

24-39.7 


\section{SUBSURFACE EXPLORATION BOREHOLE DATA BASE}

BOREHOLE:

OPERATOR:

PERMIT NO.:

LOCATION:

ELEVATION:

TOTAL DEPTH:

TYPE OF WELL:

DRILLER:

DRILLING RECORD:

CASING RECORD:

PLUGGING SCHEDULE:

STRATIGRAPHIC SUMMARY: Attached

material

\author{
B-302 \\ Sergent, Hauskins, \& Beckwith \\ Unknown \\ North Access Road \\ Not Recorded \\ $39^{\prime}$ \\ Shallow Exploratory Drilling \\ Sergent, Hauskins \& Beckwith
}

Date Started: $\quad 02 / 07 / 79$

Date Completed:

$02 / 07 / 79$

Drilling Equipment:

Truck-mounted CME-55 drill rigs powered with 6 cylinder Ford industrial engines were used in advancing test borings. The 6 cylinder engines are capable of delivering about 6,500 foot/pounds torque to the drill spindle. The spindle is advanced with twin hydraulic rams capable of exerting 12,000 pounds downward force. Drilling through soil or softer rock was performed with $61 / 2$ inch O.D., 3 1/4 inch I.D. hollow stem auger. Carbide insert teeth were used on the auger bits. Core drilling in bedrock was performed using NX size core bits with either diamond or carbide cutting faces and either air or water for drilling fluid.

Backfilled with native drilled 


\section{STRATIGRAPHIC SUMMARY}

BOREHOLE

B-302

Sand

Caliche

Dewey Lake
DEPTH INTERVAL IN FEET

0-10.4

$10.4-23$

$23-39.0$ 
BOREHOLE:

OPERATOR:

PERMIT NO.:

LOCATION:

ELEVATION:

TOTAL DEPTH:

TYPE OF WELL:

DRILLER:

DRILLING RECORD:

CASING RECORD:

PLUGGING SCHEDULE:
B-303

Sergent, Hauskins, \& Beckwith

Unknown

North Access Road

Not Recorded

$39.1^{\prime}$

Shallow Exploratory Drilling

Sergent, Hauskins \& Beckwith

Date Started: $\quad 02 / 07 / 79$

Date Completed:

$02 / 07 / 79$

Drilling Equipment:

Truck-mounted CME-55 drill rigs powered with 6 cylinder Ford industrial engines were used in advancing test borings. The 6 cylinder engines are capable of delivering about 6,500 foot/pounds torque to the drill spindle. The spindle is advanced with twin hydraulic rams capable of exerting 12,000 pounds downward force. Drilling through soil or softer rock was performed with $61 / 2$ inch O.D., 3 $1 / 4$ inch I.D. hollow stem auger. Carbide insert teeth were used on the auger bits. Core drilling in bedrock was performed using NX size core bits with either diamond or carbide cutting faces and either air or water for drilling fluid.

Backfilled with native drilled material

STRATIGRAPHIC SUMMARY: Attached 


\section{STRATIGRAPHIC SUMMARY}

BOREHOLE

B-303

Sand

Caliche

Dewey Lake
DEPTH INTERVAL IN FEET

$0-15.5$

$15.5-18$

$18-39.1$ 
BOREHOLE:

OPERATOR:

PERMIT NO.:

LOCATION:

ELEVATION:

TOTAL DEPTH:

TYPE OF WELL:

DRILLER:

DRILLING RECORD:

CASING RECORD:

PLUGGING SCHEDULE:

STRATIGRAPHIC SUMMARY: Attached

B-304

Unknown

41.5' material
Sergent, Hauskins, \& Beckwith

North Access Road

Not Recorded

Shallow Exploratory Drilling

Sergent, Hauskins \& Beckwith

Date Started: $\quad 02 / 06 / 79$

Date Completed:

02/06/79

Drilling Equipment:

Truck-mounted CME-55 drill rigs powered with 6 cylinder Ford industrial engines were used in advancing test borings. The 6 cylinder engines are capable of delivering about 6,500 foot/pounds torque to the drill spindle. The spindle is advanced with twin hydraulic rams capable of exerting 12,000 pounds downward force. Drilling through soil or softer rock was performed with $61 / 2$ inch O.D., 3 $1 / 4$ inch I.D. hollow stem auger. Carbide insert teeth were used on the auger bits. Core drilling in bedrock was performed using NX size core bits with either diamond or carbide cutting faces and either air or water for drilling fluid.

Backfilled with native drilled 


\section{STRATIGRAPHIC SUMMARY}

BOREHOLE

B-304
ROCK UNIT

DEPTH INTERVAL IN FEET

Sand

Caliche

Sand
$0-20.6$

$20.6-33$

$33-41.5$ 
BOREHOLE:

OPERATOR:

PERMIT NO.:

LOCATION:

ELEVATION:

TOTAL DEPTH:

TYPE OF WELL:

DRILLER:

DRILLING RECORD:

CASING RECORD:

PLUGGING SCHEDULE:

STRATIGRAPHIC SUMMARY: Attached

\section{B-305}

Unknown

41' material
Sergent, Hauskins, \& Beckwith

North Access Road

Not Recorded

Shallow Exploratory Drilling

Sergent, Hauskins \& Beckwith

Date Started: $\quad 02 / 06 / 79$

Date Completed:

$02 / 06 / 79$

Drilling Equipment:

Truck-mounted CME-55 drill rigs powered with 6 cylinder Ford industrial engines were used in advancing test borings. The 6 cylinder engines are capable of delivering about 6,500 foot/pounds torque to the drill spindle. The spindle is advanced with twin hydraulic rams capable of exerting 12,000 pounds downward force. Drilling through soil or softer rock was performed with $61 / 2$ inch O.D., 3 1/4 inch I.D. hollow stem auger. Carbide insert teeth were used on the auger bits. Core drilling in bedrock was performed using NX size core bits with either diamond or carbide cutting faces and either air or water for drilling fluid.

Backfilled with native drilled 


\section{STRATIGRAPHIC SUMMARY}

BOREHOLE

B-305
ROCK UNIT

DEPTH INTERVAL IN FEET

Sand

Caliche

Sand

Caliche

Sand
$0-14.5$

$14.5-19.5$

$19.5-25.5$

25.5-33

33-41 
BOREHOLE:

OPERATOR:

PERMIT NO.:

LOCATION:

ELEVATION:

TOTAL DEPTH:

TYPE OF WELL:

DRILLER:

DRILLING RECORD:

CASING RECORD:

PLUGGING SCHEDULE:

STRATIGRAPHIC SUMMARY: Attached

\section{B-306}

$38^{\prime}$

material
Sergent, Hauskins, \& Beckwith

Unknown

North Access Road

Not Recorded

Shallow Exploratory Drilling

Sergent, Hauskins \& Beckwith

Date Started: $\quad 02 / 02 / 79$

Date Completed:

$02 / 02 / 79$

Drilling Equipment:

Truck-mounted CME-55 drill rigs powered with 6 cylinder Ford industrial engines were used in advancing test borings. The 6 cylinder engines are capable of delivering about $6,500 \mathrm{foot} /$ pounds torque to the drill spindle. The spindle is advanced with twin hydraulic rams capable of exerting 12,000 pounds downward force. Drilling through soil or softer rock was performed with $61 / 2$ inch O.D., 3 1/4 inch I.D. hollow stem auger. Carbide insert teeth were used on the auger bits. Core drilling in bedrock was performed using NX size core bits with either diamond or carbide cutting faces and either air or water for drilling fluid.

Backfilled with native drilled 


\section{STRATIGRAPHIC SUMMARY}

BOREHOLE

B-306
ROCK UNIT

DEPTH INTERVAL IN FEET

Sand

Caliche

Santa Rosa
$0-7.3$.

$7.3-18$

18-38 


\section{SUBSURFACE EXPLORATION BOREHOLE DATA BASE}

BOREHOLE:

OPERATOR:

PERMIT NO.:

LOCATION:

ELEVATION:

TOTAL DEPTH:

TYPE OF WELL:

DRILLER:

DRILLING RECORD:

CASING RECORD:

\section{B-307}

Sergent, Hauskins, \& Beckwith

Unknown

Railroad

Not Recorded

$40^{\prime}$

Shallow Exploratory Drilling

Sergent, Hauskins \& Beckwith

Date Started: $\quad 02 / 01 / 79$

Date Completed:

$02 / 01 / 79$

Drilling Equipment:

Truck-mounted CME-55 drill rigs powered with 6 cylinder Ford industrial engines were used in advancing test borings. The 6 cylinder engines are capable of delivering about 6,500 foot/pounds torque to the drill spindle. The spindle is advanced with twin hydraulic rams capable of exerting 12,000 pounds downward force. Drilling through soil or softer rock was performed with $61 / 2$ inch O.D., 3 1/4 inch I.D. hollow stem auger. Carbide insert teeth were used on the auger bits. Core drilling in bedrock was performed using NX size core bits with either diamond or carbide cutting faces and either air or water for drilling fluid.

Backfilled with native drilled material

STRATIGRAPHIC SUMMARY: Attached 


\section{STRATIGRAPHIC SUMMARY}

BOREHOLE

B-307
ROCK UNIT

Caliche

Sand

Gatuna

Dewey Lake
DEPTH INTERVAL IN FEET

$0-5.6$

5.6-11.1

11.1-17.1

$17.1-40$ 


\section{SUBSURFACE EXPLORATION BOREHOLE DATA BASE}

BOREHOLE:

OPERATOR:

PERMIT NO.:

LOCATION:

ELEVATION:

TOTAL DEPTH:

TYPE OF WELL:

DRILLER:

DRILLING RECORD:

CASING RECORD:

PLUGGING SCHEDULE:

STRATIGRAPHIC SUMMARY： Attached

B-308

Railroad

$39.9^{\prime}$ material
Sergent, Hauskins, \& Beckwith

Unknown

Not Recorded

Shallow Exploratory Drilling

Sergent, Hauskins \& Beckwith

Date Started: $\quad 02 / 01 / 79$

Date Completed: $\quad 02 / 01 / 79$

Drilling Equipment:

Truck-mounted CME-55 drill rigs powered with 6 cylinder Ford industrial engines were used in advancing test borings. The 6 cylinder engines are capable of delivering about $6,500 \mathrm{foot} /$ pounds torque to the drill spindle. The spindle is advanced with twin hydraulic rams capable of exerting 12,000 pounds downward force. Drilling through soil or softer rock was performed with $61 / 2$ inch O.D., 3 1/4 inch I.D. hollow stem auger. Carbide insert teeth were used on the auger bits. Core drilling in bedrock was performed using NX size core bits with either diamond or carbide cutting faces and either air or water for drilling fluid.

Backfilled with native drilled 


\section{STRATIGRAPHIC SUMMARY}

B-308

Caliche

Gatuna

Dewey Lake
$0-10.5$

$10.5-19.5$

19.5-39.9 


\section{SUBSURFACE EXPLORATION BOREHOLE DATA BASE}

BOREHOLE:

OPERATOR:

PERMIT NO.:

LOCATION:

ELEVATION:

TOTAL DEPTH:

TYPE OF WELL:

DRILLER:

DRILLING RECORD:

CASING RECORD:

PLUGGING SCHEDULE:

\section{B-309}

Sergent, Hauskins, \& Beckwith

Unknown

South Access Road

Not Recorded

39.4'

Shallow Exploratory Drilling

Sergent, Hauskins \& Beckwith

Date Started: $\quad 02 / 02 / 79$

Date Completed:

$02 / 02 / 79$

\section{Drilling Equipment:}

Truck-mounted CME-55 drill rigs powered with 6 cylinder Ford industrial engines were used in advancing test borings. The 6 cylinder engines are capable of delivering about $6,500 \mathrm{foot} /$ pounds torque to the drill spindle. The spindle is advanced with twin hydraulic rams capable of exerting 12,000 pounds downward force. Drilling through soil or softer rock was performed with $61 / 2$ inch O.D., 3 $1 / 4$ inch I.D. hollow stem auger. Carbide insert teeth were used on the auger bits. Core drilling in bedrock was performed using NX size core bits with either diamond or carbide cutting faces and either air or water for drilling fluid.

Backfilled with native drilled material

STRATIGRAPHIC SUMMARY: Attached 


\section{STRATIGRAPHIC SUMMARY}

BOREHOLE

B-309
ROCK UNIT

Sand

Caliche

Gatuna

Santa Rosa

Dewey Lake
DEPTH INTERVAL IN FEET

$0-5.6$

$5.6-14$

$14-19.8$

$19.8-22$

22-39.4 


\section{REFERENCE LIST}

\section{Major References}

Beauheim, R.L. Interpretations of Single-Well Hydraulic Tests Conducted At and Near the Waste Isolation Pilot Plant (WIPP) Site, 1983-1987. SAND87-0039, Sandia National Laboratories, Albuquerque, New Mexico. December 1987.

Beauheim, R.L., et al. Basic Data Report for Borehole Cabin Baby-1 Deepening and Hydrologic Testing, Waste Isolation Pilot Plant (WIPP) Project, Southeastern New Mexico. WTSD-TME-020, U.S. Department of Energy, Albuquerque, New Mexico. December 1983.

Dennehy, K.F. Results of Hydrologic Tests and Water-Chemistry Analysis, Wells H-6A, H-6B, and H-6C, at the Proposed Waste Isolation Pilot Plant Site, Southeastern New Mexico. U.S. Geological Survey Water-Resources Investigation Report 82-8. 1982.

Freeland, M.H. Basic Data Report for Borehole DOE-1, Waste Isolation Pilot Plant (WIPP) Project, Southeastern .New Mexico. TME 3159, U.S. Department of Energy, Albuquerque, New Mexico. 1982.

Gonzales, M.M. Compilation and Comparison of Test-Hole Location Surveys in the Vicinity of the Waste Isolation Pilot Plant (WIPP) Site. SAND88-1065, Sandia National Laboratories, Albuquerque, New Mexico. February 1989.

Gonzalez, D.D. Hydrogeochemical Parameters of Fluid-Bearing Zones in the Rustler and Bell Canyon Formation. SAND83-0210. March 1983.

Hydro Geo Chem, Inc. WIPP Hydrology Program, Waste Isolation Pilot Plant, SENM, Hydrologic Data Report \#1. SAND85-7206, Hydro Geo Chem, Inc., Tucson, Arizona. September 1985.

INTERA Technologies, Inc., and Hydro Geo Chem, Inc. WIPP Hydrology Program, Waste Isolation Pilot Plant, Southeastern New Mexico, Hydrologic Data Report \#2. SAND857263, INTERA Technologies, Inc., Austin, Texas and Hydro Geo Chem, Inc., Tucson, Arizona. December 1985.

INTERA Technologies, Inc. WIPP Hydrology Program, Waste Isolation Pilot Plant, Southeastern New Mexico, Hydrologic Data Report \#3. SAND86-7109, INTERA Technologies Inc., Austin, Texas. June 1986. 
Mercer, J.W., and B.R. Orr. Interim Data Report on the Geohydrology of the Proposed Waste Isolation Pilot Plant Site Southeast New Mexico. USGSOFR-79-98, U.S. Geological Survey, Denver, Colorado. 1979.

Mercer, J.W., et al. Results of Hydrologic Tests and Water Chemistry Analyses, Wells H-4A, $\mathrm{H}-4 \mathrm{~B}, \mathrm{H}-4 \mathrm{C}$ at the Proposed Waste Isolation Pilot Plant Site, Southeastern New Mexico. U.S. Geological Survey Water-Resources Investigations 81-36, U.S. Geological Survey, Albuquerque, New Mexico. 1981.

Mercer, J.W., and R.P. Snyder. Basic Data Report for Drillholes H-17 and H-18 (Waste Isolation Pilot Plant-WIPP). SAND89-0204, Sandia National Laboratories, Albuquerque, New Mexico. February 1990.

Mercer, J.W., and R.P. Snyder. Basic Data Report for Drillholes H-14 and H-15 (Waste Isolation Pilot Plant-WIPP). SAND89-0202, Sandia National Laboratories, Albuquerque, New Mexico. February 1990.

Potash Mine or Bureau of Land Management (SUNDRY NOTICES AND REPORTS ON WELLS)

SAND 85-7206, September 1985, WIPP Hydrology Program Waste Isolation Pilot Plant, SENM, Hydrologic Data Report \#1

Saulnier, G.J., et al. WIPP Hydrology Program, Waste Isolation Pilot Plant, Southeastern New Mexico, Hydrologic Data Report \#4. SAND86-7166, INTERA Technologies Inc., Austin, Texas. February 1987.

Sergent, Hauskins \& Beckwith, Volume I, Subsurface Exploration \& Laboratory Testing, Plant Site, Waste Isolation Pilot Plant, Bechtel Job No. 12484

Seward, P.D. Abridged Borehole Histories for the Waste Isolation Pilot Plant (WIPP) Studies. SAND82-0080, Sandia National Laboratories, Albuquerque, New Mexico. January 1982.

U.S. DOE/WIPP 89-006, February 1989, Well History for the Water Quality Sampling Program

U.S. DOI Geological Survey, Open-File Report 78-592

Winstanley, D.J. and R.C. Carrasco. Annual Hydrologic Data Report, 1985-1986. DOE-WIPP 86-004, WIPP Project, Carlsbad, New Mexico. October 1986. 


\section{Minor References}

Black, S.R. Basic Data Report Borehole WIPP-12 Deepening, Waste Isolation Pilot Plant (WIPP) Project, Southeastern New Mexico. TME 3148, U.S. Department of Energy, Albuquerque, New Mexico. 1982.

Cauffman, T.L., et al. Ground Water Flow Modeling of the Culebra Dolomite-Volume II:Data Base. SAND89-7068/2, INTERA Technologies, Inc., Austin, Texas. October 1990.

Griswold, G.B., and V.C. McWhirter. Interpretation of Wireline Geophysical Logs ERDA No. 9 Stratigraphic Test Borehole DOE WIPP Site, Eddy County, New Mexico. SAND807119, Telecote Corporation, Albuquerque, New Mexico. February 1981.

Memo from Jery W. Mercer, January 22, 1991

Mercer, J.W., and R.P. Snyder. Basic Data Report for Drillhole H-12 (Waste Isolation Pilot Plant-WIPP). SAND89-0201, Sandia National Laboratories, Albuquerque, New Mexico. January 1990.

Mercer, J.W., and R.P. Snyder. Basic Data Report for Drillhole H-16 (Waste Isolation Pilot Plant-WIPP). SAND89-0203, Sandia National Laboratories, Albuquerque, New Mexico. January 1990.

Mercer, J.W. Basic Data Report for Drillholes at the H-11 Complex (Waste Isolation Pilot Plant-WIPP). SAND89-0200, Sandia National Laboratories, Albuquerque, New Mexico. May 1990.

SAND 79-0268, Basic Data Report for Drillhole AEC-7

SAND 79-0268, Basic Data Report for Drillhole AEC-8

Sandia National Laboratories, and U.S. Geological Survey. Basic Data Report for Drillhole WIPP-30 (Waste Isolation Pilot Plant-WIPP). SAND79-0284, Sandia National Laboratories, Albuquerque, New Mexico. April 1980.

Sandia National Laboratories, and U.S. Geological Survey. Basic Data Report for Drillhole WIPP-28 (Waste Isolation Pilot Plant-WIPP). SAND79-0282, Sandia National Laboratories, Albuquerque, New Mexico. August 1979. 
Sandia National Laboratories, and U.S. Geological Survey. Basic Data Report for Drillhole WIPP-29 (Waste Isolation Pilot Plant-WIPP). SAND79-0283, Sandia National Laboratories, Albuquerque, New Mexico. September 1980.

Sandia National Laboratories, and D'Appolonia Consulting Engineers. Basic Data Report for Drillhole WIPP-14 (Waste Isolation Pilot Plant-WIPP). SAND82-1783, Sandia National Laboratories, Albuquerque, New Mexico. August 1982.

Sandia National Laboratories, and D'Appolonia Consulting Engineers. Basic Data Report for Deepening of Drillhole WIPP-13 (Waste Isolation Pilot Plant-WIPP). SAND82-1880, Sandia National Laboratories, Albuquerque, New Mexico. October 1982.

Sandia National Laboratories, and U.S. Geological Survey. Basic Data Report for Drillhole WIPP-26 (Waste Isolation Pilot Plant-WIPP). SAND79-0280, Sandia National Laboratories, Albuquerque, New Mexico. August 1979.

Sandia National Laboratories, and U.S. Geological Survey. Basic Data Report for Drillhole WIPP-27 (Waste Isolation Pilot Plant-WIPP). SAND79-0281, Sandia National Laboratories, Albuquerque, New Mexico. October 1979.

Sandia National Laboratories, and U.S. Geological Survey. Basic Data Report for Drillhole WIPP-21 (Waste Isolation Pilot Plant-WIPP). SAND79-0277, Sandia National Laboratories, Albuquerque, New Mexico. March 1980.

Sandia National Laboratories, and U.S. Geological Survey. Basic Data Report for Drillhole WIPP-22 (Waste Isolation Pilot Plant-WIPP). SAND79-0278, Sandia National Laboratories, Albuquerque, New Mexico. March 1980.

Sandia National Laboratories, and U.S. Geological Survey. Basic Data Report for Drillhole WIPP-19 (Waste Isolation Pilot Plant-WIPP). SAND79-0276, Sandia National Laboratories, Albuquerque, New Mexico. March 1980.

Sandia National Laboratories, and U.S. Geological Survey. Basic Data Report for Drillhole WIPP-18 (Waste Isolation Pilot Plant-WIPP). SAND79-0275, Sandia National Laboratories, Albuquerque, New Mexico. March 1980.

Sandia National Laboratories, and D'Appolonia Consulting Engineers. Basic Data Report for Drillhole WIPP-12 (Waste Isolation Pilot Plant-WIPP). SAND82-2336, Sandia National Laboratories, Albuquerque, New Mexico. October 1982.

Stensrud, W.A. Reconfiguration of H-4c, H-5c, and H-6c. Communication to Dan Winstanley, Westinghouse Electric. August 1986. 
Stensrud, W.A., et al. WIPP Hydrology Program, Waste Isolation Pilot Plant, Southeastern New Mexico, Hydrologic Data Report \#7. SAND88-7014, INTERA Technologies Inc., Austin, Texas. November 1988.

Stensrud; W.A., et al. WIPP Hydrology Program, Waste Isolation Pilot Plant, Southeastern New Mexico, Hydrologic Data Report \#8. SAND89-7056, INTERA Technologies Inc., Austin, Texas. April 1990.

Stensrud, W.A., et al. WIPP Hydrology Program, Waste Isolation Pilot Plant, Southeastern New Mexico, Hydrologic Data Report \#6. SAND87-7166, INTERA Technologies Inc., Austin, Texas. May 1988.

Stensrud, W.A., et al. WIPP Hydrology Program, Waste Isolation Pilot Plant, Southeastern New Mexico, Hydrologic Data Report \#5. SAND87-7125, INTERA Technologies Inc., Austin, Texas. October 1987.

TME-020, Basic Data Report for Borehole Cabin Baby-1 Deepening and Hydraulic Testing

USGS OFR 81-469, Geologic Data for Borehole ERDA-9, Eddy County, New Mexico

USGS OFR 81-488, Geologic Data for Borehole ERDA-9, Eddy County, New Mexico 